\title{
Multi-Antenna Configuration Modeling for Massive MIMO V2I
}

\author{
Andreas Pfadler
}

\author{
Supervised by \\ Prof. Dr. Lluís Jofre Roca
}

Final Project Report for the

Master's degree in Telecommunications Engineering (MET)

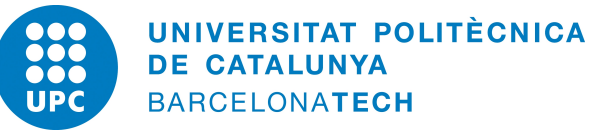

Dep. Communications and Signal Processing

Technical University of Catalonia

Barcelona, Spain

03-2018 



\title{
Title of the thesis: Multi-Antenna Configuration Modeling for
}

\author{
Massive MIMO V2I \\ Author: Andreas Pfadler \\ Advised by: Prof. Dr. Lluís Jofre Roca
}

\section{Abstract}

Vehicle communications are now emerging towards the 5th Generation Wireless Systems (5G). Shortly, an explosive increase in the number of connected vehicles is expected. This communication is not only for car to car safety reasons, but also for communicating with the environment concerning entertainment and internet. Larger data rates as provided by current Long Term Evolution (LTE) systems are required. Energy efficiency is becoming more and more important as part of the green communication for the $5 \mathrm{G}$ network. Consequently, there is a growing research interest in massive Multiple Input Multiple Output (MIMO) antenna systems providing higher channel capacity, spectral and energy efficiency compared to conventional MIMO without beamforming. Also conventional MIMO needs to be investigated for Vehicle to Infrastructure (V2I) communication, since the evolution towards the 5G network is slow hence interim solutions are essential. Furthermore, the impact on the channel capacity for different inter-element spacing for antennas on top of the car is examined.

In order to study the impact of different multi-antenna geometries and MIMO modalities in both vehicle and fixed Base Station (BS), a quality modeling of a realistic scenario is required. The analysis is based on a numerical simulated model of an urban scenario, emulating as a case of study a portion of the city of Barcelona in a realistic environment, comprising of a massive MIMO base station and a car at various positions on a trajectory. Several systems and configurations are compared by means of the channel eigenvalues and capacity. On top of the vehicle one, two or four monopole antennas are used for the investigation. Corresponding at the $\mathrm{BS}$ one, two or four patch antennas are used for conventional MIMO and 64 patch antennas grouped as one, two or four beams for massive MIMO.

The following key findings are found for V2I communication in the presented work. For all massive MIMO configurations the interference for other users is highly reduced. Furthermore, since there is a huge increase of received power for the vehicle without losing the heterogeneity of the eigenvalues, this leads to high channel capacity with less transmitted power. For a Signal to Noise Ratio (SNR) of $12 \mathrm{~dB}$ with conventional MIMO 4x4 almost $170 \%$ more average channel capacity can be gained with respect to a Single Input Single Output (SISO) in Line of Sight (LOS). For Non Line of Sight (NLOS) it is even $190 \%$, which is very close to the ideal limit. For different inter-element spacing, the channel capacity also depends on the amount of elements in the car. The higher the number of elements, the higher is the achievable channel capacity, but larger inter-element distance is necessary to obtain the optimal performance. 


\section{Acknowledgements}

Here I would like to express my sincere gratitude to my teacher, family, colleagues, and friends.

First and foremost, I would like to express my deep and sincere gratitude to my supervisor Professor Lluís Jofre Roca, for giving me the opportunity to do research and providing valuable guidance throughout my final thesis. His great sense of humor, vision, and dynamism have deeply inspired me. It was a privilege and honor to work and study under his direction. I am highly thankful for what he has offered me.

Special thanks to Dr. Manish Rana and my brother Dr. Thomas Pfadler, who mentored me step by step towards research and advised me with their expertness and perceptiveness. I have learned many things from them: like professional experience, methodologies of scientific working and analytically and critical reasoning. I am sure all these skills will benefit me throughout my private and professional life.

The authors thanks go to his colleague Christian Ballesteros Sánchez, who has put considerable time and effort into his comments on the entire project.

Finally I would also like to thank my family and friends who supported, and encouraged me all the time. 


\section{Contents}

1 Introduction $\quad 1$

1.1 Research Background and Literature Review . . . . . . . . . . . . . . 1

1.1.1 Propagation Environment . . . . . . . . . . . . . 1

1.1.2 Massive MIMO Base Station . . . . . . . . . . . . . . . . . 2

1.1.3 Vehicle Antenna Configuration ............. . . 4

1.2 Motivation . . . . . . . . . . . . . . . . . 4

1.3 Objectives and Milestones . . . . . . . . . . . . . . . 5

1.4 Contributions . . . . . . . . . . . . . . . . . 7

1.5 Thesis Organization . . . . . . . . . . . . . . 8

2 Theoretical Background $\quad 9$

2.1 Electromagnetic Modeling of the Channel:
Ray Tracing . . . . . . . . . . . . . . . . . . . . . . . . 9 9

2.2 MIMO Channel . . . . . . . . . . . . . . . . . . . . 10

2.2.1 Basic Parameters . . . . . . . . . . . . . . . 10

2.2.2 Massive MIMO and Beamforming . . . . . . . . . . . . . 12

2.2 .3 Correlation . . . . . . . . . . . . . . . . 13

3 System Model $\quad 14$

3.1 Urban Environment . . . . . . . . . . . . . . . . . . . . . . . . 14

3.2 Vehicle Multi-antenna Setup . . . . . . . . . . . . . . . . 16

3.3 Base Station . . . . . . . . . . . . . . . . . . . . . . 18

3.3.1 Validation of Propagation Environment . . . . . . . . . 21

4 Methodology 23

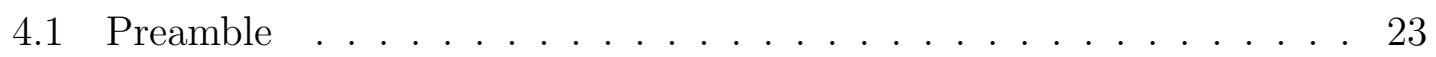

4.2 Method . . . . . . . . . . . . . . . . . . 24

4.3 Numerical Analysis . . . . . . . . . . . . . . . . . . . . 25

4.4 Data Analysis . . . . . . . . . . . . . . . . . 25

5 Simulation Results and Analysis $\quad 27$

5.1 Conventional MIMO . . . . . . . . . . . . . . . . . . 27

5.2 Massive MIMO . . . . . . . . . . . . . . . . . . . . . . 30

5.3 Different Inter-element Spacing for Conventional MIMO . . . . . . . . 37

6 Conclusion $\quad 39$ 
A Appendix

A.1 Conference Paper: URSI $2017 \ldots \ldots \ldots \ldots$. . . . . . . 43

A.2 Conference Paper: EuCAP $2018 \ldots \ldots \ldots \ldots$. . . . . . . . 48

A.3 Conference Paper: ADAPTIVE $2018 \ldots \ldots \ldots \ldots$. . . . . . 54

A.4 Submitted Journal Paper: Special Issue . . . . . . . . . . . . . . 59

A.5 Submitted Conference Paper: EuMW $2018 \ldots \ldots \ldots$. . . . . . . 67

A.6 Research Budget . . . . . . . . . . . . . . . . . . . . . . . 73 


\section{List of Figures}

1.1 Generic model of a V2I scenario. . . . . . . . . . . . . . 5

1.2 Gantt Chart with main objectives and milestones of the master project. 7

2.1 Fermat's principle states that a ray always takes the fastest time of travel; hence the path ABC. $[17$, pg.1] . . . . . . . . . . 10

2.2 The reflected ray (left) from a flat surface involving two distinct mediums and the diffracted rays (right) from an edge. [18] . . . . . . . . 10

2.3 A planar two dimensional array. [23, pg.202] . . . . . . . . . . . 12

2.4 Correlation for two dipoles with changing inter-element spacing and three distinct angle of arrival. [25] . . . . . . . . . . . . . . . 13

3.1 The Scenario with the intersection of Passeig de Sant Joan and Avinguda Diagonal in Barcelona, Spain. . . . . . . . . . . . . . . 15

3.2 Antenna gain on top of a vehicle with side view (blue) and front view (green) at $3.6 \mathrm{GHz}$ in FEKO [28]. . . . . . . . . . . . . . . . . 17

3.3 Three different vehicle antenna setups with one, two, and four monopole antennas with an inter-element spacing $d$ between the antennas. . . . 18

3.4 Antenna gain of a monopole antenna on top of a vehicle at $3.6 \mathrm{GHz}$ in FEKO [28] . . . . . . . . . . . . . . . . . . . . . . . . . . . . . 18

3.5 Three different configurations of the BS with 64 patch antennas for massive MIMO communication. . . . . . . . . . . . . . . . . . . 19

3.6 Antenna gain for one massive beam $\left(B S_{1}\right)$ towards reference point $P_{1}$ (top) and towards the second reference point $P_{2}$ (bottom) at $3.6 \mathrm{GHz}$ in FEKO [28]. . . . . . . . . . . . . . . . . . . . . . . . . .

3.7 Power map of the incoherent power received at a height of 1.42 meters and with a resolution of 1 meter in WinProp [27]. . . . . . . . . . . . 21

3.8 Received power for massive MIMO towards $P_{2}$ and conventional SISO

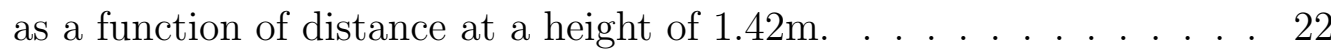

4.1 Overview of the procedure, its four steps, and the used programs for the numerical V2I communications research model. . . . . . . . . 23

5.1 Channel capacity for conventional MIMO in LOS. . . . . . . . . . . . 27

5.2 Average channel capacity for conventional MIMO over the entire trajectory (mean SNR of $13 \mathrm{~dB}$ ) and LOS. . . . . . . . . . . . . . 28

5.3 Channel capacity for conventional MIMO in NLOS. . . . . . . . . . 29

5.4 Average channel capacity for conventional MIMO over the entire trajectory (mean SNR of $8 \mathrm{~dB}$ ) and NLOS. . . . . . . . . . . 30

5.5 Received power for massive MIMO towards reference point $P_{1}$ at each position on the trajectory. . . . . . . . . . . . . . . 31 
5.6 Received power for massive MIMO towards reference point $P_{2}$ at each position on the trajectory. . . . . . . . . . . . . . . . . 32

5.7 Frobenius normalized eigenvalues for Multi-user massive Multiple Input Multiple Output (MU-mMIMO) 2x2 and 4x4 at each position on the trajectory with beamforming towards $P_{1} \ldots \ldots . \ldots 33$

5.8 Normalized eigenvalues for MU-mMIMO $2 \times 2$ and $4 \times 4$ at each position on the trajectory with beamforming towards $P_{2} \ldots \ldots$. . . . . . . 34

5.9 Channel capacities for massive MIMO towards reference point $P_{1}$ at each position on the trajectory. . . . . . . . . . . . . 35

5.10 Channel capacities for massive MIMO towards reference point $P_{2}$ at each position on the trajectory. . . . . . . . . . . . . . 35

5.11 Overview of the massive configuration average channel capacities and their corresponding average SNR when transmitting towards $P_{1}$ and $P_{2}$ with a constant overall transmit power of $0 \mathrm{dBm}$ at the BS. . . . . 36

5.12 Average channel capacity for different inter-element spacing for MIMO 2x2 and $4 \times 4$ with respect to the SISO channel capacity in percent and a

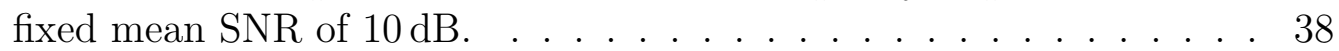




\section{List of Tables}

1.1 Overview of all investigated MIMO modalities with $\mathbf{x}_{x}$ as the BS input and $\mathbf{y}_{x}$ as the receiving vehicle output, and the channel $\mathbf{H}$ in between. . . . . . . . . . . . . . . . . 3

3.1 Overview of BS configuration for LOS and NLOS of the investigated MIMO modalities. . . . . . . . . . . . . . . . . . . . 14

3.2 Settings of the propagation path interactions in WinProp [27]. . . . . 16

3.3 Overview of the transmitter and receiver configuration for conventional MIMO. . . . . . . . . . . . . . . . . . . . . 19

3.4 Overview of the transmitter and receiver for massive MIMO. . . . . 20

3.5 Overview of used overall transmitted power. . . . . . . . . . . . 20

A.1 Research Budget . . . . . . . . . . . . . . . . . 73 


\section{Glossary}

\section{Acronyms}

3D Three-dimensional

5G 5th Generation Wireless Systems

AF Array Factor

BS Base Station

ECC Electronic Communications Committee

GTD Geometrical Theory of Diffraction

LOS Line of Sight

LTE Long Term Evolution

MIMO Multiple Input Multiple Output

MLFMM Multilevel Fast Multipole Method

MoM Method of Moments

MU-mMIMO Multi-user massive Multiple Input Multiple Output

MU-mSISO Multi-user massive Single Input Single Output

NLOS Non Line of Sight

PIFAs Planar Inverted F Antennas

SISO Single Input Single Output

SNR Signal to Noise Ratio

TDMA Time Division Multiple Access

UTD Uniform Theory of Diffraction

V2I Vehicle to Infrastructure

V2V Vehicle to Vehicle

V2X Vehicle to Everything 



\section{Chapter 1}

\section{Introduction}

This chapter presents an introduction to the thesis. The background and literature review of a V2I wireless communication system and its three main parts are given; propagation environment, the Massive MIMO Base Station and the vehicle antenna configuration. The motivation for this research project is stated followed by its main contributions. Finally, the thesis organization is presented.

\subsection{Research Background and Literature Review}

During the past few years, automotive and telecommunications industries have put their focus on Vehicle to Everything (V2X) applications due to the increasing interest of users and manufacturers in a more comfortable and secure driving. V2X communication will also be a part of the $5 \mathrm{G}$; therefore, developers and researchers are looking for solutions to connect vehicles with the infrastructure. New approaches are needed in this research field, such as the introduction of MIMO systems. Also, technological innovations such as massive MIMO systems for the BS are nowadays a promising candidates.

Regulation entities have defined some standards, with the allocation of spectrum, as a starting point in order to develop new technologies. On one hand, the Electronic Communications Committee (ECC) and the European Union defined a 30 megahertz band at $5.9 \mathrm{GHz}$, which was finally integrated in the IEEE $802.11 \mathrm{p}$ standard for V2X applications [1]; on the other hand, the automotive industry is trying to follow another approach, focusing on lower carrier bands with better propagation properties for mobile communications: between $3.4-3.8 \mathrm{GHz}$ [2]. In this work, the latter band is chosen for the design of the model since it has not been researched previously and consequently there is a lack of specifications on it for connected cars.

In this work, the analysis will be centred on the physical layer for several antenna configurations mounted on a car in an urban environment. Due to the complexity of the system, numerical methods are required and this implies the use of simulation tools. Before entering into details, a more general overview of a V2I wireless communication system and its three main parts is presented subsequently.

\subsubsection{Propagation Environment}

The propagation environment for a V2I communication can be divided into three main areas; urban, suburban, and highway propagation area [3]. Each of these areas 
are distinguishable through their characteristics. In practice, it is not always simple to differentiate between these areas because their borders are not clearly separated. However a division is reasonable due to their varying path-loss exponents and similarly their mean delay spreads, which fluctuates strongly in each area. Suburban areas are usually composed of low-rise building with greater separation between the buildings, more green spaces and less traffic. On a highway, a high doppler shift is expected through the fast traffic. Urban areas are described by high buildings, many cars and therefore a lot of users; hence a high-level of interference is likely. The speed of the users is not too high there, consequently the doppler shift is smaller in comparison to suburban and highway environment. There are different approaches for the modeling of V2I communication. Some approaches focus on the signal processing at the cost of simplifying the electromagnetic modeling while others concentrate on the electromagnetic modeling of the channel; in this work the latter is prioritized. Henceforth, doppler shift is not retained into the estimation of the channel capacity at different still positions of the car. The channel capacity in this work is always the gross channel capacity for a carrier frequency of 3.6 gigahertz in bits per second per hertz for still positions of the car. Furthermore, the V2I communication is investigated in an urban environment which is the most challenging in terms of interference and the employment of a massive MIMO BS is reasonable [4]. Most of the time, the communication between the vehicle and the BS might be NLOS, but as cells are getting smaller, LOS communication is getting more important.

In order to model the wave propagation in an urban environment, ray tracing as a numerical method is used, while in the related work [5], the channel is approximated with static models. For more details on statistical modelling of massive MIMO channels, the reader is encouraged to refer to [6].

\subsubsection{Massive MIMO Base Station}

Massive MIMO is an arising new technology, where "massive" refers to a large number of antennas at the BS. Table 1.1 shows the overview of all investigated modalities. It is distinguished between conventional $M \times N$ MIMO, where $M$ refers to the number of BS transmitting units and $N$, to the vehicle receiving ones, and massive configurations, where a total amount of $Q$ elements are distributed in a set of $M$ modules of $P$ elements each $(Q=M \times P)$.

The Multi-user massive Single Input Single Output (MU-mSISO) BS is composed of $Q$ antenna elements with steering capabilities (one beam towards the receiver). It can be seen as a SISO communication with beamforming at the BS. MU-mSISO is normally called massive MIMO in literature, as defined in [7] and it is different from conventional MIMO techniques [8]. Subdividing the massive BS into $M$ modules instead of working as a single antenna, the MU-mMIMO configurations allow to introduce $M$ ports feeding a set of $P$ antennas each. Henceforth, the channel matrix $\mathbf{H}$ consists of $M \times N$ entries. M beams are steered towards the receiver with $\mathrm{N}$ single antennas, which is similar to the conventional MIMO case but using beamforming.

What are the reasons for using a massive number of antennas at the BS? Specifically, the throughput for the vehicle can be enhanced by using a massive MIMO BS and, on the other hand, it reduces the interference for other users. The application of massive MIMO and their highly concentrated beam towards a vehicle is worthwhile only if the car position is known precisely and the update of the steered beam 
Table 1.1: Overview of all investigated MIMO modalities with $\mathbf{x}_{x}$ as the BS input and $\mathbf{y}_{x}$ as the receiving vehicle output, and the channel $\mathbf{H}$ in between.

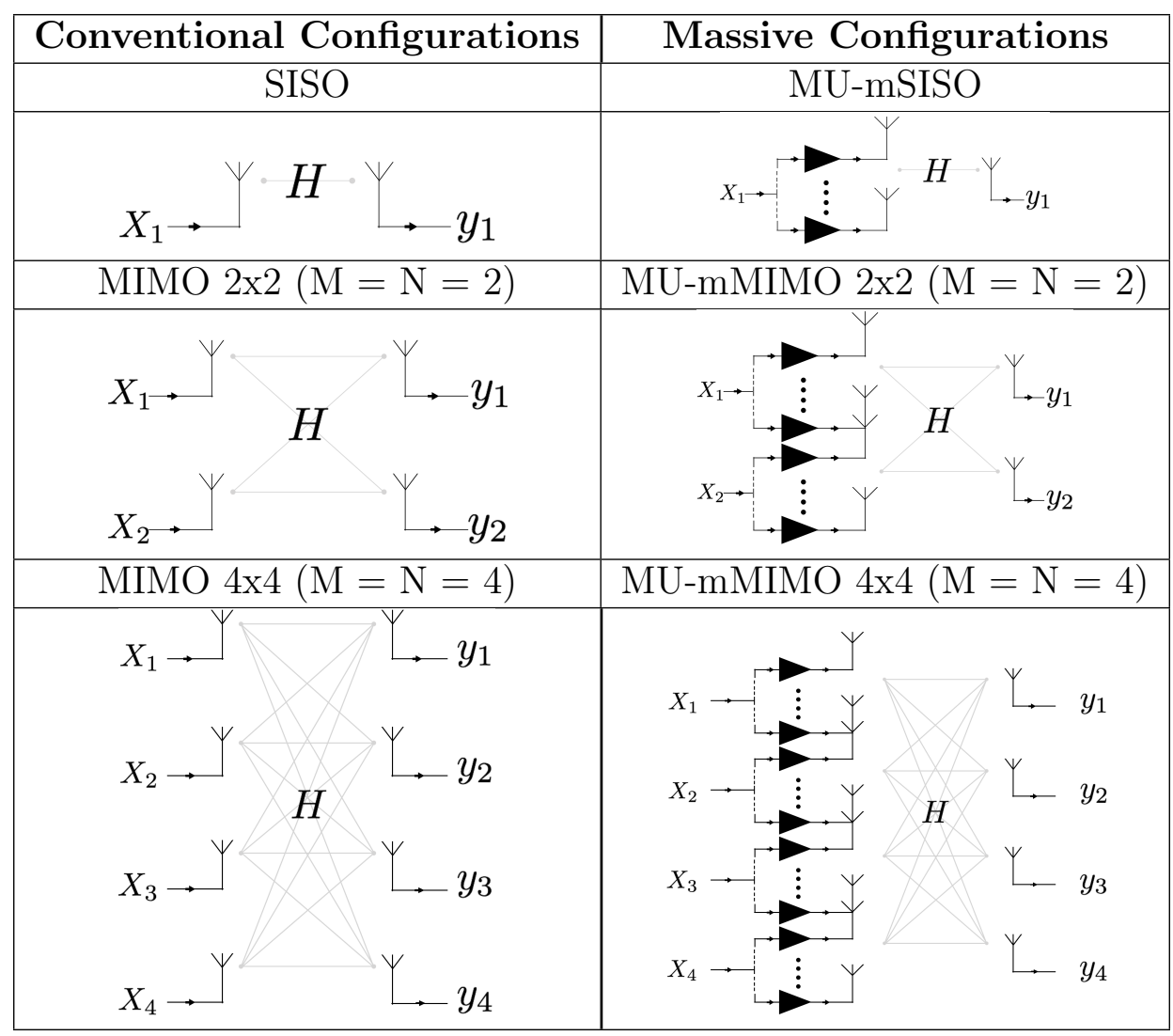


is fast enough. Henceforth, the BS is receiving a pilot signal from the car and is able to estimate its position. This precise estimation of a vehicle location is challenging. In the worst case, the car will not receive anything from the BS. For instance, pilot contamination can lead to inaccuracies in the estimation of the car locations. This happens when the BS can not distinguish among pilot sequences from several users [9].

Theoretical models [10] suggest that when the antenna elements increase towards infinity, the region of concentration of the beamforming could become arbitrarily small until reaching the diffraction limit (in the order of the wavelength). However at this limit, it is also shown that the pilot contamination is a limiting factor for the accurate estimation of vehicle location. Mutual coupling is another source of inaccuracy in the estimation of the car location, because it changes the array radiation pattern [11]. Furthermore, there are physical constrains due to the size of the antenna array.

\subsubsection{Vehicle Antenna Configuration}

These days, vehicle antennas are settled onto the top of the car under a sharkfin plastic cover. This plastic cover restricts the physical size of the antenna in a compact way due to aesthetic reasons [12]. Recent research primarily concentrated on antenna designs under this physical limitation by proposing e.g. a Vivaldi antenna [13]. Other works investigated MIMO LTE antennas in a shark-fin cover by using Planar Inverted F Antennas (PIFAs) [14]. In the context of this research project, initial approaches were focused on planar patch antennas [15]. Patch antennas can be placed directly on top of the car without the need of using a shark-fin cover. The spacing between the antenna elements can be increased and an increment of channel capacity is expected due to the reduction of correlation [16]. A drawback of using patch antennas is that the directivity of a planar patch antenna on the rooftop of a car pointing towards the zenith. Therefore, the more efficient monopole based antennas for V2I communications on the rooftop of the vehicle are used.

\subsection{Motivation}

Fig. 1.1 illustrates a generic model of a V2I communication system. The general operation of the entire system would consist on a first step, in which the potential users send a pilot signal that allows the BS to identify and locate them spatially. With this information, in a second stage, the BS radiates into the coverage area with a focusing strategy depending on the specific configuration. It illuminates the different areas where the users (vehicles) are located. Due to the angular distribution of the BS field, some other regions may be reached by the signal and an interference level may be noticed outside the area of interest.

Research is done at the physical level. The research question, which needs to be answered is: What is the optimal antenna configuration on top of the vehicle for V2I communication? Here, current technologies, i.e. MIMO need to be investigated but also new arising innovations like massive MIMO have to be examined. Therefore, the motivation of this Master project is to compose a novel numerical simulation model as a combination of different simulation tools. Such a model allows the evaluation of different geometries and types of antennas. Specifically, the estimation of number 


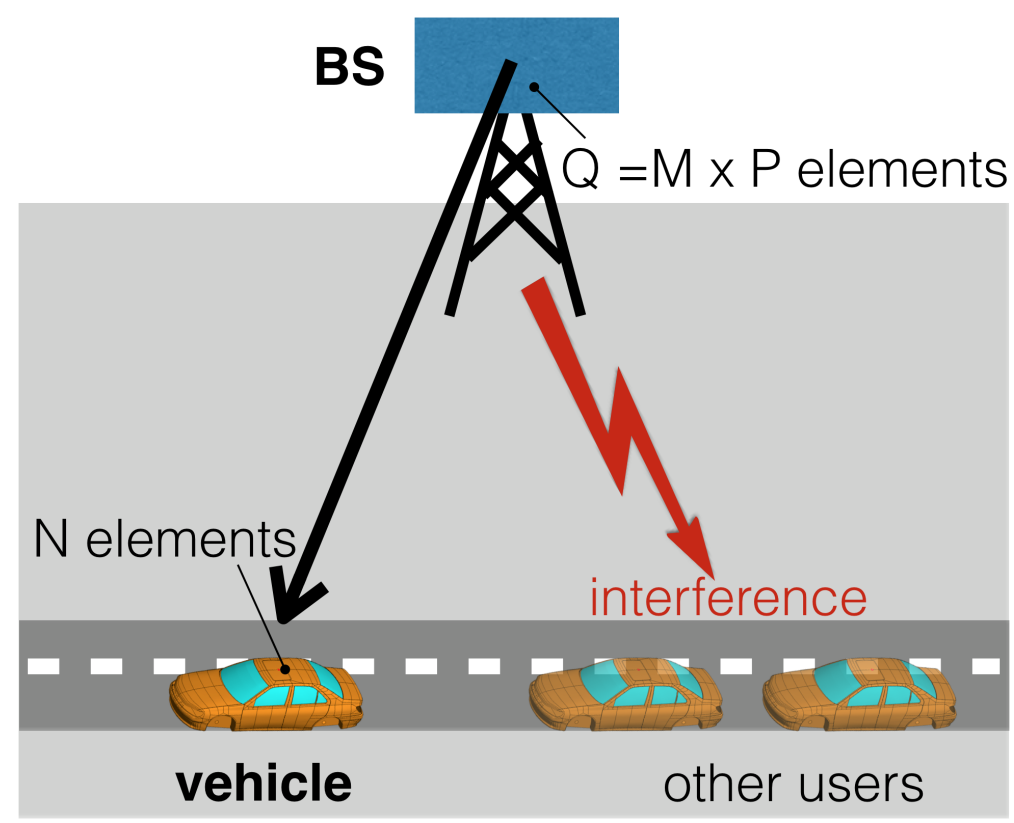

Fig. 1.1: Generic model of a V2I scenario.

of antenna elements needed for an efficient communication on the top of the car. Trade-off for more antennas implicate more cost and also aesthetic issues depending on the antenna size, geometry and type of antenna.

In order to reach these objectives, a model is needed. Here, the modelling is realized with commercial simulation tools, which are based on numerical methods. WinProp is used for the simulation of the wave propagation and FEKO for electromagnetic radiation pattern. One, two and four antennas placed on top of the car are compared with different inter-element spacing. A proper model of a vehicle is essential to get a realistic radiation pattern of an antenna pattern on the rooftop of the car.

\subsection{Objectives and Milestones}

Fig. 1.2 shows five objectives and corresponding to these goals five important milestones of this work. The main objectives of the thesis are stated as follows:

- Objective 1: Investigate a MIMO V2I communication for a simplified scenario.

- Objective 2: Investigate different massive MIMO V2I modalities for distinct car antenna configurations for a realistic scenario.

- Objective 3: Optimizing the inter-element distance of the antennas on top of the car for an efficient MIMO communication.

- Objective 4: Advanced investigation of Objective 2 with a rigorous electromagnetic channel modeling.

- Objective 5: Developing, designing, and testing a 5G vehicle antenna. 
Corresponding to the objectives, five milestones are achieved:

- Milestone I: Capacity calculation for a simple scenario considering a realistic model of a car.

- URSI conference paper 2017: "Antenna Geometry Optimization for MIMOConnected Cars". (see Appendix A.1)

- Milestone II: Capacity of the channel for different car and BS configurations in a realistic scenario.

- EuCAP conference paper 2018: "Multi-antenna Configuration Modeling for Massive MIMO V2I". (see Appendix A.2)

- Milestone III: Vehicle antenna footprint optimization.

- ADAPTIVE conference paper 2018: "Vehicle Antenna Footprint Optimization for Efficient MIMO V2X Communications". (see Appendix A.3)

- Milestone IV: Capacity figures for massive MIMO.

- Submitted special issue on wave propagation and antennas journal 2018: "Propagation-based Performance of Massive MIMO Antenna Configurations for V2I UrbanCommunications". (see Appendix A.4)

- Milestone V: Fabrication and measuring of an UWB ice-cream cone antenna.

- Submitted EuMW conference paper 2018: "5G Vehicle MIMO Antenna Capacity Based on a Rigorous Electromagnetic Channel Modeling". (see Appendix A.5) 


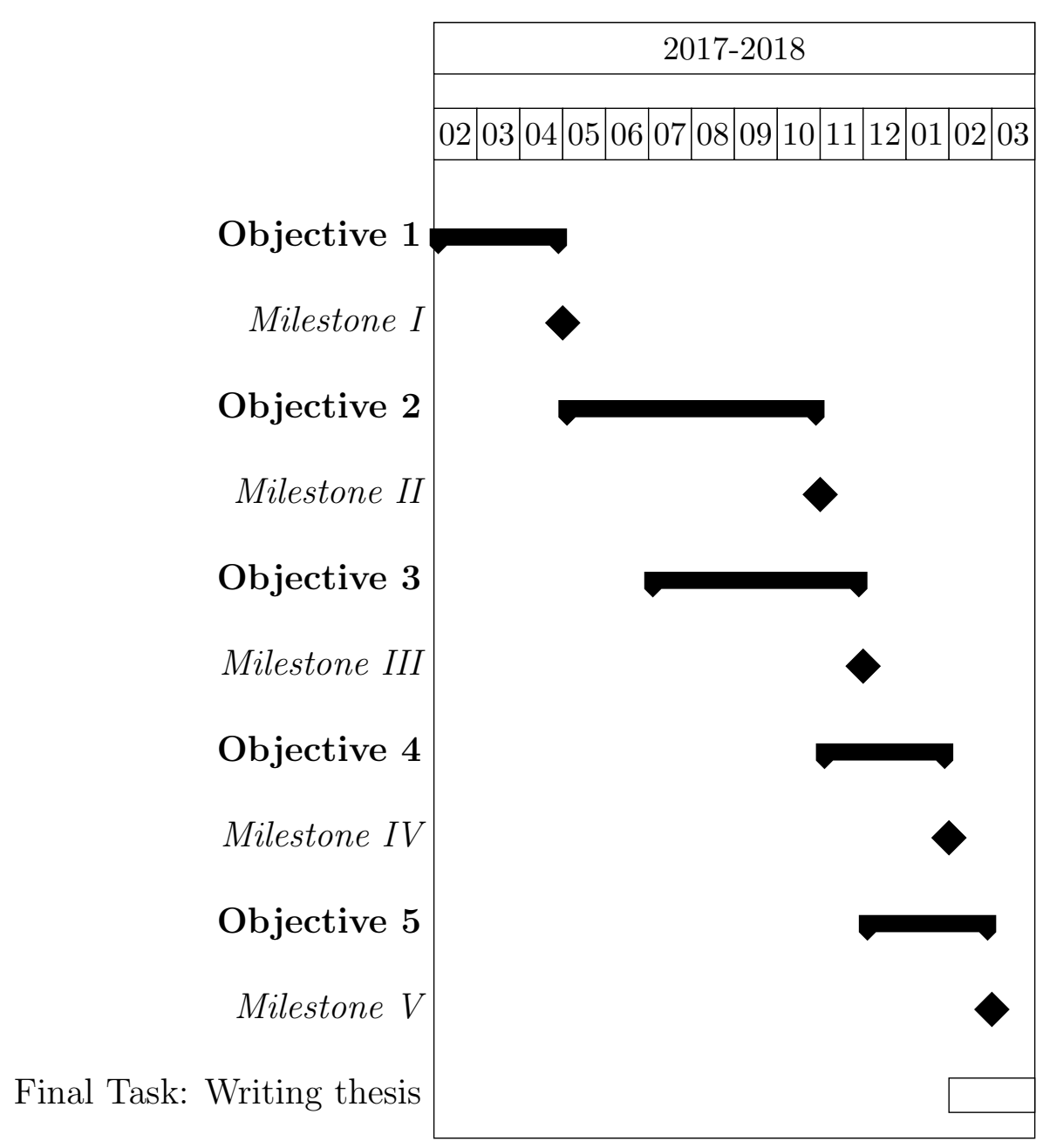

Fig. 1.2: Gantt Chart with main objectives and milestones of the master project.

\subsection{Contributions}

The key contributions of the thesis are summarized as follows:

- Proposal of a novel numerical simulation model for V2I communication.

- Evaluation of different MIMO modalities for V2I communication in an urban environment.

- Evaluation of different massive MIMO modalities for V2I communication in an urban environment.

- Comparison between conventional and massive MIMO BS for V2I communication.

- The impact on the channel capacity by changing the inter-element spacing of the antenna array on top of a vehicle. 


\subsection{Thesis Organization}

The thesis is organized as follows:

- Chapter 2 gives a theoretical background.

- Chapter 3 describes the complete system, including all elements composing the model (i.e. urban environment, antenna elements, vehicle and BS), for which the channel will be identified.

- Chapter 4 presents the methodology and numerical tools which are used to evaluate the simulation results.

- Chapter 5 depicts the results and analysis of conventional MIMO, massive MIMO, and conventional MIMO with different inter-element spacing.

- Chapter 6 presents the conclusion and points out future directions in this research area. 


\section{Chapter 2}

\section{Theoretical Background}

The goal is to model the complex V2I system including the modeling of the physical channel for all MIMO modalities and the channel capacity calculations for conventional MIMO for different inter-element spacing. In order to reach such an environment the ray tracing method appears to be the most realistic one and in fact the one used on the recent full 3-dimensional tools. This chapter is organized as follows: First, ray tracing is discussed. Then the MIMO channel is defined, followed by some theoretical background for massive MIMO and beamforming systems. Finally, the correlation and its impact on the channel capacity is addressed.

\subsection{Electromagnetic Modeling of the Channel: Ray Tracing}

In 1654, Pierre de Fermat postulated the Fermat's principle stating that a ray takes always the shortest time to travel from one point to another no matter what sort of reflection the ray belongs to. After completing the theory the classical geometrical optics were created. [17, pg.3]

Fig. 2.1 shows Fermat's principle for three different paths: AB'C, ABC, and $\mathrm{AB}$ " $\mathrm{C}$ between the source A and sink $\mathrm{C}$. The wave propagates from A to B and gets reflected towards $\mathrm{C}$ because path $\mathrm{ABC}$ is the shortest way of travel. That was the beginning of modern ray tracing algorithms. Rays can be basically classified into the following types: direct rays, reflected rays, and diffracted rays [18]. The simplest ray is the direct ray or LOS ray, here the ray goes directly from the source to the sink. Reflected rays are the rays which interact with different mediums. Fig. 2.2 depicts on the left an incident ray and its reflected ray, which follows the law of reflection and the Fresnel's equations for the magnitude of the reflected ray. Fig. 2.2 illustrates on the right one incident ray and its corresponding diffracted rays, which are distributed on a Keller cone. Diffraction methods are distinctly more complex than the reflected rays. Therefore, different methods were proposed. In 1960's Joseph Bishop Keller developed Geometrical Theory of Diffraction (GTD) based on Fermat's principle and the law of diffraction was expressed. It stays that the angle of the incident ray is equal to the angle of the diffracted ray. The limit of GTD are the shadow boundaries, where the results are not continuous across. The method of Uniform Theory of Diffraction (UTD) helped to overcome this issue and was proposed by Kouyoumjian and Pathak [19]. These methods are commonly used 


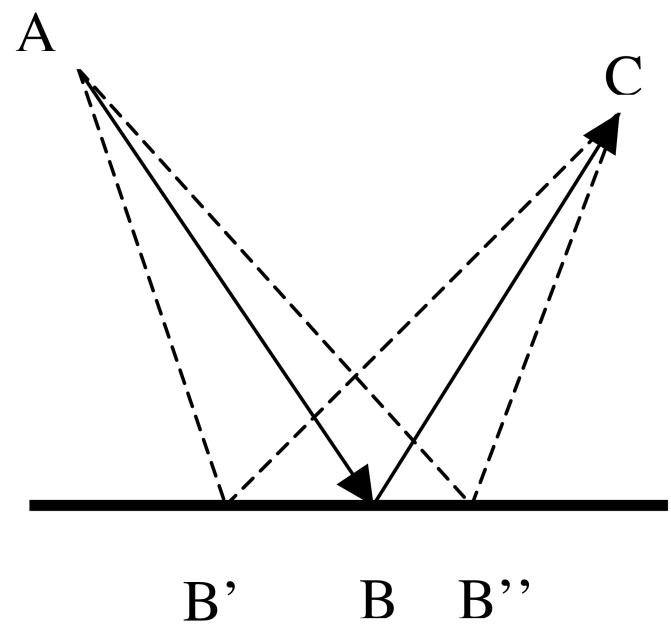

Fig. 2.1: Fermat's principle states that a ray always takes the fastest time of travel; hence the path ABC. [17, pg.1]
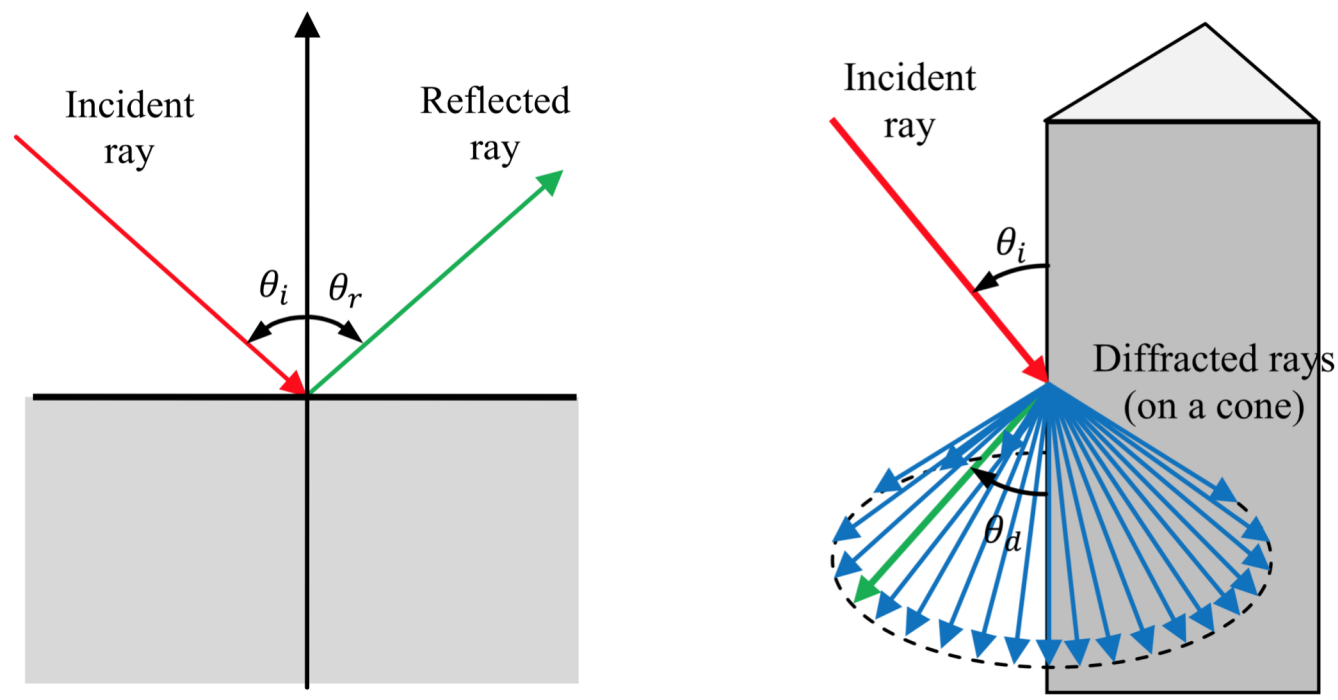

Fig. 2.2: The reflected ray (left) from a flat surface involving two distinct mediums and the diffracted rays (right) from an edge. [18]

in order to obtain the diffraction coefficients. The same methods namely GTD and UTD for diffraction and the Fresnel's equations for reflections are used by the wave simulation program (WinProp) for this work.

\section{$2.2 \quad$ MIMO Channel}

In this section the basic concepts of a MIMO channel, beamforming in the context of massive MIMO, and correlation are introduced briefly.

\subsubsection{Basic Parameters}

"As in the study of any type of system, a communication channel is usually viewed in terms of its possible inputs, its possible outputs, and a description of how the input affects the output. This description is usually probabilistic." [20, pg.8] Ray 
tracing describes in a numerical way the relation between the input vector $\vec{x}(t)$ and the output vector $\vec{y}(t)$ by calculating the channel matrix $\mathbf{H}$.

$$
\boldsymbol{y}(t)=\boldsymbol{H} \boldsymbol{x}(t)
$$

The role of the channel is essential to understand the limitations of a communication system and its variability must be analyzed to reach trustworthy conclusions. The channel matrix includes several parameters to consider when it is calculated. It is given by the so called Friis Transmission equation for ideal free-space matched antennas without polarization mismatch:

$$
\Gamma(\vec{r})=\frac{P_{R}}{P_{T}}=D_{T}(\theta, \phi) \cdot D_{R}(\theta, \phi) \cdot\left(\frac{c_{0}}{4 \pi r f}\right)^{2},
$$

where, $D_{R}(\theta, \phi)$ and $D_{T}(\theta, \phi)$ is the Three-dimensional (3D) directivity of both the receiving and transmitting antennas, respectively, $c_{0}$ is the speed of light in freespace, $\mathrm{f}$ is the carrier frequency, and $\mathrm{r}$ is the distance between transmitter and receiver. Obviously, frequency affects the path loss. This is the main reason to work at the lowest value possible, when the antenna aperture is small. Choosing the 3.6 $\mathrm{GHz}$ band would suppose an improvement respect to the band around $5.9 \mathrm{GHz}$. The channel transfer function can be expressed as follows:

$$
h_{i j}(\vec{r})=\Gamma(\vec{r}) e^{-j k \vec{r}}
$$

where $k$ is the wave number. The channel matrix is given by summing up all propagation paths from $\mathrm{i}$-th transmitter to $\mathrm{j}$-th receiver.

$$
H=\sum_{i, j=1}^{n} h_{i j}
$$

In information theory, the channel capacity is defined as the highest information rate that can be succeeded with indiscriminately small error probability [21]. Therefore, this work is based on ideal coding, working with the maximum channel capacity and not with outage channel capacity. The capacity for a MIMO system may be obtained as [22]:

$$
C=\log _{2}\left(\operatorname{det}\left[I_{N r}+\frac{P_{R} \cdot\|H\|_{F}\|H\|_{F}^{*}}{P_{N}}\right]\right),
$$

where, $H$ is the $N r \times N t$ channel matrix, $P_{R}$ and $P_{N}$ are received and noise power, respectively, $I_{N r}$ is the identity matrix, and (.)* denotes the transpose conjugate. The channel matrix is normalized with the Frobenius norm:

$$
\|H\|_{F}=\left(\sum_{i, j=1}^{n}\left|h_{i j}\right|^{2}\right)^{1 / 2}
$$

Additionally, the expression can be defined as a function of the eigenvalues $\lambda_{i}$ with $i$-th element of a matrix resulting from the product between $H$ and its transpose conjugate [22]:

$$
C=\sum_{i=1}^{N} \log _{2}\left(1+\frac{P_{T} \cdot \lambda_{i}}{P_{N}}\right)
$$


A new parameter is defined as $N=\min \left(N_{T}, N_{R}\right)$, which is the number of non-null eigenvalues (matrix rank) or, in terms of communications, the maximum number of simultaneous information flows that can be sent in a MIMO system. Furthermore, it is common to asses the correlation of the channel matrix by its channel condition number. The condition number can be calculated with the largest and lowest eigenvalue with the following equation [6]:

$$
C N(d b)=20 \log _{10} \frac{\lambda_{\max }\left(\|H\|_{F}\right)}{\lambda_{\max }\left(\|H\|_{F}\right)},
$$

where $\lambda_{\max }$ and $\lambda_{\min }$ correspond to the maximum and the minimum eigenvalue of the normalized channel matrix, respectively. A small channel condition number indicates low correlation and consequently higher channel capacity. The ideal channel condition number would be $0 \mathrm{~dB}$ and in any case $C N \geq 0 \mathrm{~dB}$.

\subsubsection{Massive MIMO and Beamforming}

Massive MIMO is commonly referred to receiving pilot signals from the users, using linear pre-coding, and transmitting with all BS antennas combined as a massive beam towards the users with Time Division Multiple Access (TDMA). In this work the position of the vehicle is known. Therefore, the time of arrival for the BS can be calculated in order to steer the beam towards the user positions. Fig. 2.3 illustrates a sketch of a planar two dimensional array with $A$ elements in the y-axis and $B$ elements in the x-axis. In order to steer the beam, the coefficients $\alpha_{a b}$, the electrical angles $\Psi_{x}$ and $\Psi_{x}$ need to be selected. The coefficients define the pattern shape, while the phases are steering the pattern shape towards a certain direction. The array coefficients may be canonically distributed uniformly, triangularly or binomial. For this work the coefficients are selected to be uniform, due to the highest directivity which can be obtained. The phases may be calculated with the corresponding $\theta$ and $\phi$ angle, which points towards the desired direction. This can be done by calculating

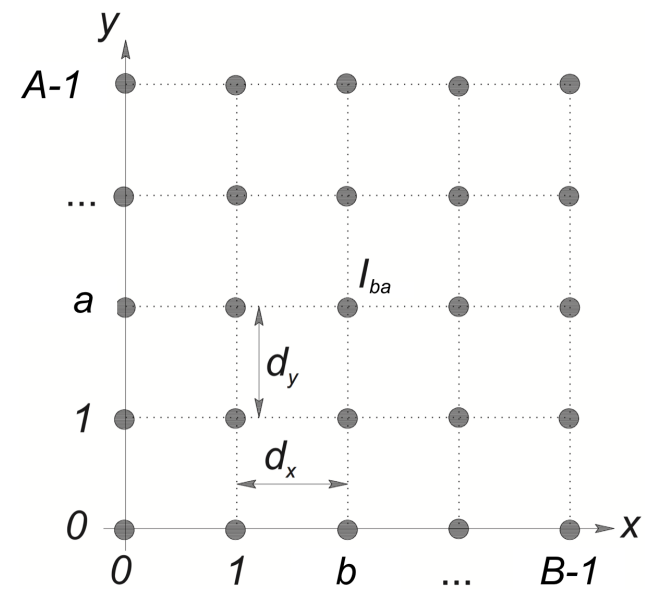

Fig. 2.3: A planar two dimensional array. [23, pg.202]

the Array Factor (AF), i.e., for a planar two dimensional array:

$$
A F\left(\Psi_{x}, \Psi_{y}\right)=\sum_{b=0}^{B} \sum_{a=0}^{A} \alpha_{a b} \cdot e^{j b \Psi_{x}} \cdot e^{j a \Psi_{y}} .
$$


The electrical angles $\Psi_{x}$ and $\Psi_{x}$ may be calculated by:

$$
\begin{gathered}
\Psi_{x}=k \cdot d_{x} \cdot \sin (\theta) \cdot \cos (\phi)+\alpha_{x} \\
\Psi_{y}=k \cdot d_{y} \cdot \sin (\theta) \cdot \cos (\phi)+\alpha_{y},
\end{gathered}
$$

where, $\alpha_{x}$ and $\alpha_{y}$ is the relative phase into the $\mathrm{x}$ - and y-direction, respectively.

[23, pg.202-203]

\subsubsection{Correlation}

The distance between antennas has a direct impact on the channel capacity through the correlation between the antenna elements. This fading correlation was researched by Shiu et al. [24]. The smaller the angle spread becomes, the higher is the correlation and the lower is the responding channel capacity [22]. Therefore, a higher inter-element spacing is needed for small angle spreads. Fig. 2.4 shows the correlation for three different kinds of possible arrival angles for two dipoles with varying inter-element spacing. Furthermore, mutual coupling between the antennas is another penalty on the channel capacity, which is not studied in this work.

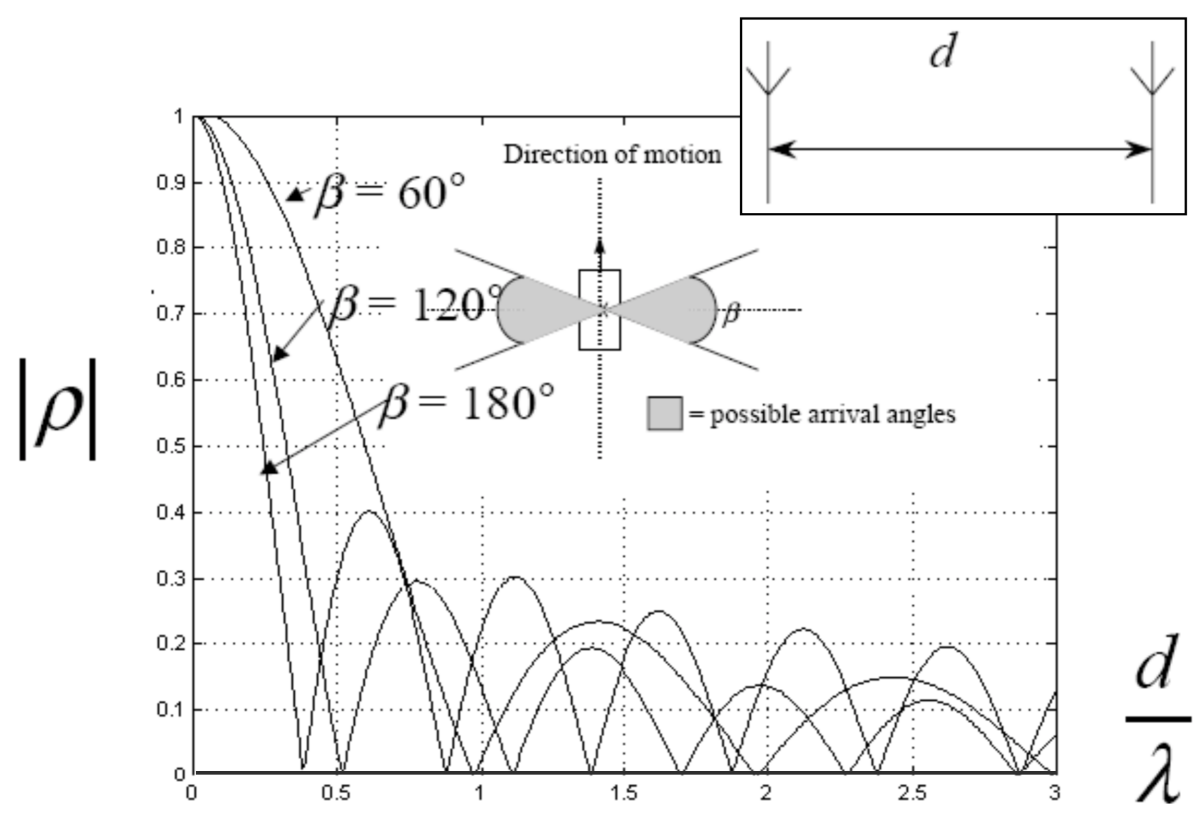

Fig. 2.4: Correlation for two dipoles with changing inter-element spacing and three distinct angle of arrival. [25] 


\section{Chapter 3}

\section{System Model}

Once the theoretical background is stated, the system model needs to be elaborated and created. It is presented in following chapter and consists of the urban environment, BS configuration and vehicle mobile user with a multi-antenna setup. Finally, a validation of the propagation environment is demonstrated.

\subsection{Urban Environment}

In order to obtain a more realistic approach for the analysis of a car in an urban area, a portion of a real city is chosen to simulate a V2I environment. In particular, Fig. 3.1 depicts the intersection Avinguda Diagonal with Passeig de Sant Joan in the city of Barcelona, corresponding to the Eixample. It is a district characterized by its rectangular shapes and almost 90 degrees corners. Fig. 3.1a shows the satellite view of Google Maps. Fig. 3.1b depicts the simplified replication in WinProp. The environment is comprised of a ground plane made of asphalt, five buildings, three of them with courtyards, traffic lights and trees. The height of all buildings is 18 meter and the BS is placed on the top of one building at height of 21 meters, 3 meters above the rooftop. There are two positions for the BS on top of the building, one for LOS and the other for NLOS communication. Table 3.1 illustrates an overview of the investigated MIMO modalities for LOS and NLOS V2I communication and its corresponding $B S_{C}$, where $\mathrm{C}$ is referred to the configuration of logical inputs and outputs. For LOS six modalities are investigated: three conventional MIMO and three massive MIMO; for NLOS there are three conventional MIMO modalities. As represented in Fig. 3.1b, a reference trajectory ( $143.4 \mathrm{~m}$ long sampled every $0.1 \mathrm{~m}$ ) is defined for the moving car, with three characteristic points: $P_{1}$ (at $45 \mathrm{~m}$ on the trajectory), corner point $(C P)($ at $70 \mathrm{~m})$ and $P_{2}$ (at $\left.85 \mathrm{~m}\right)$. The BS steers its beam to follow the trajectory in a discrete way, where $P_{1}$ and $P_{2}$ represent the two specific

Table 3.1: Overview of BS configuration for LOS and NLOS of the investigated MIMO modalities.

\begin{tabular}{|l|l|l|l|}
\cline { 2 - 4 } \multicolumn{1}{c|}{} & \multicolumn{3}{c|}{ BS configurations } \\
\cline { 2 - 4 } \multicolumn{1}{c|}{} & $B S_{1}$ & $B S_{2}$ & $B S_{3}$ \\
\hline Line of Sight (LOS) & SISO & MIMO 2x2 & MIMO 4x4 \\
& MU-mSISO & MU-mMIMO 2x2 & MU-mMIMO4x4 \\
\hline Non Line of Sight (NLOS) & SISO & MIMO 2x2 & MIMO 4x4 \\
\hline
\end{tabular}




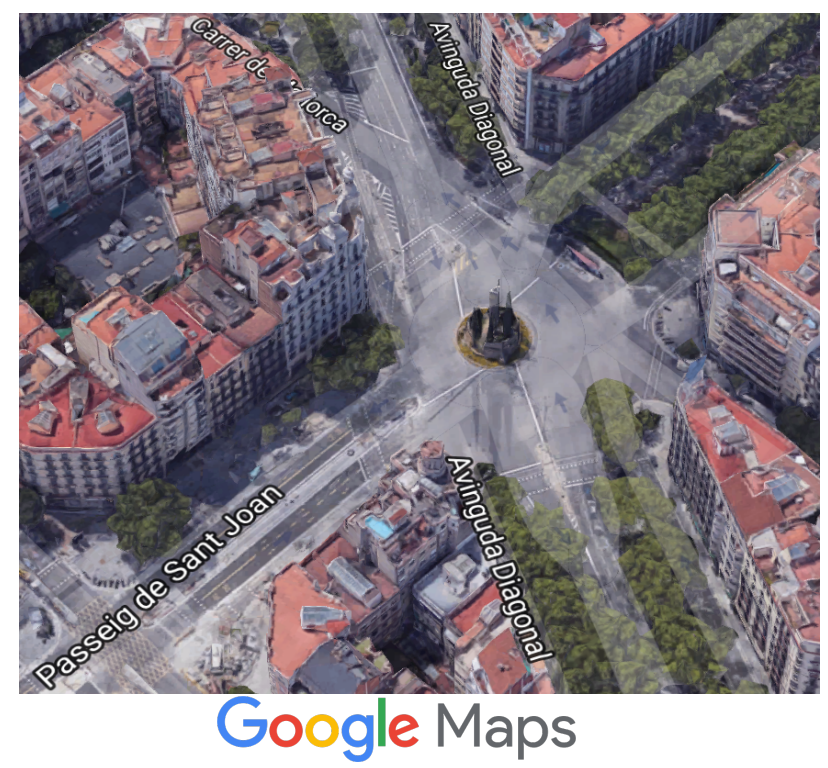

(a) Satellite image from Google Maps [26].

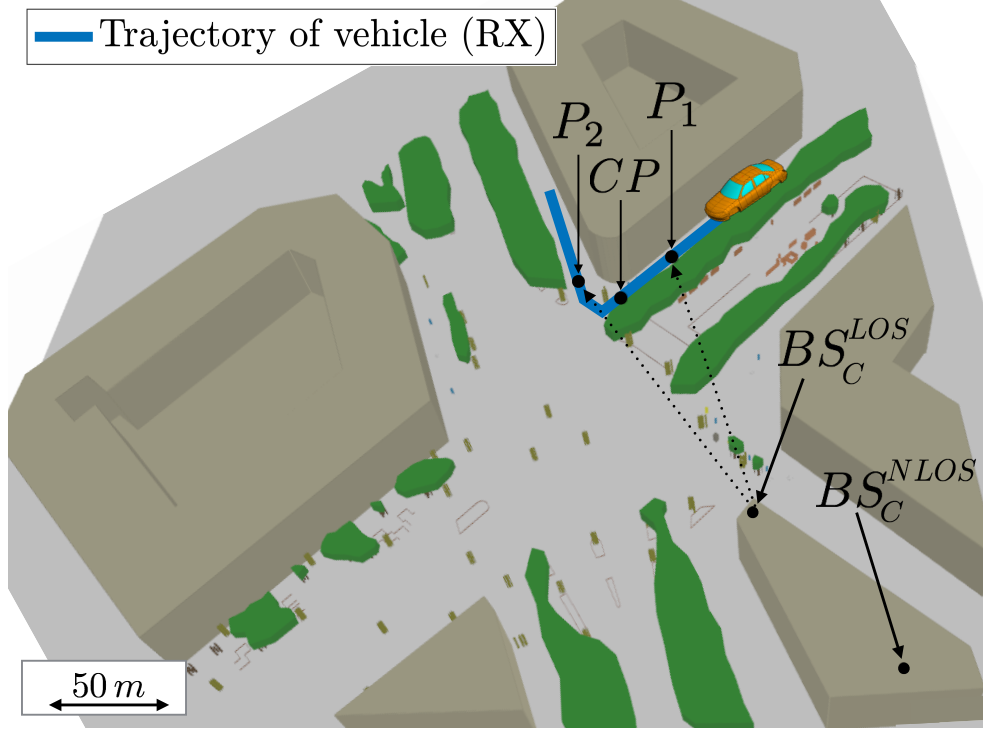

(b) Simplified model in WinProp [27].

Fig. 3.1: The Scenario with the intersection of Passeig de Sant Joan and Avinguda Diagonal in Barcelona, Spain.

points at which the car is expected to be located. $C P$ represents the closest point to the BS which it is situated on the corner of the street. Therefore, 1434 still positions of the V2I communication are considered. The contemplation of the entire trajectory instead of just the area close to the reference points allows the observation of interference for other users, i.e., the vehicle driving ahead or behind the user. In addition the obtained channel capacity can be averaged over the trajectory in order to get more convincing results. The distance between $B S_{C}^{L O S}$ and the reference point $P_{1}, C P$, and $P_{2}$ is 108,87 , and 94 meter, respectively.

All buildings are modeled as 3D objects with material properties that influence the reflection of the rays on their surface. On the other hand, trees are made of wooden trunk and vegetation top, which let the rays to pass at the cost of additional attenuation. Table 3.2 shows the selected settings for the path interactions. The 
Table 3.2: Settings of the propagation path interactions in WinProp [27].

\begin{tabular}{|l|l|}
\hline Propagation path interactions & \\
\hline \hline Max. number of transmissions & 1 \\
\hline Max. number of reflections & 3 \\
\hline Max. number of diffraction & 2 \\
\hline
\end{tabular}

chosen settings have a strong influence on the simulation time. More propagation path interactions increase the simulation effort. Especially, the maximum number of diffraction is costly due to its calculation complexity. The maximum number of transmissions refers to the number of transmission through material interactions that are allowed in a single interaction chain. One transmission might be enough for an almost LOS communication. One transmission is needed for the main stem of a tree, which causes an interaction through wood. The number of maximum reflections and diffraction is selected to be three and two, respectively. For those given settings, WinProp considers up to 20 different paths to each receiver position on the trajectory.

\subsection{Vehicle Multi-antenna Setup}

There are certain requirements for the antenna configuration on top of the car, as already discussed in Section 1.1.3. First an antenna type needs to be found. Fig. $3.2 \mathrm{a}$ shows the radiation pattern of a monopole, and Fig. $3.2 \mathrm{~b}$ for a patch antenna, both on top of the vehicle. In comparison it can be concluded that the monopole antenna still has $0 \mathrm{dBi}$ in horizontal plane $\left(\theta=90^{\circ}\right)$, whereas the patch antenna has $-4 \mathrm{dBi}$. This characteristic is important for Vehicle to Vehicle (V2V) communication. Even so V2V does not lie in the focus of this work it still needed to be considered. Furthermore, another disadvantage of the patch antenna is that the antenna gain is smaller by $10 \mathrm{~dB}$ comparing the front view with the side view at the horizontal plane due to the different pattern of the E- and H-plane, which may cause larger weighting for some rays depending on the relative position with respect to the BS. Also the main gain is pointing towards the zenith, which is not energy efficient. Considering this point, the monopole antenna shows better characteristics, because the maximum gain is pointing towards the expected angle of arrival and not towards the sky as the patch antenna does. In this work monopole antennas are used. The geometry is selected to be in a line, because the antenna systems have to be compact and fit, i.e. into a shark-fin cover.

Three different configurations are considered for the antennas on the car rooftop: one, two, and four monopole antennas. Fig. 3.3 depicts the vehicle with all antenna setups. The inter-element spacing $d$ is fixed to half of the wavelength $(4.15 \mathrm{~cm}$ at $3.6 \mathrm{GHz}$ ) for all simulations with exception of the investigation of different interelement spacing for conventional MIMO in Section 5.3. In order to obtain a realistic radiation pattern of a monopole antenna for V2I communications, the vehicle needs to be considered. Henceforth, the car body is assumed to be made of metal and the windows of laminated glass. Fig. 3.4 depicts the 3-dimensional antenna gain for one monopole antenna on top of the vehicle model. 


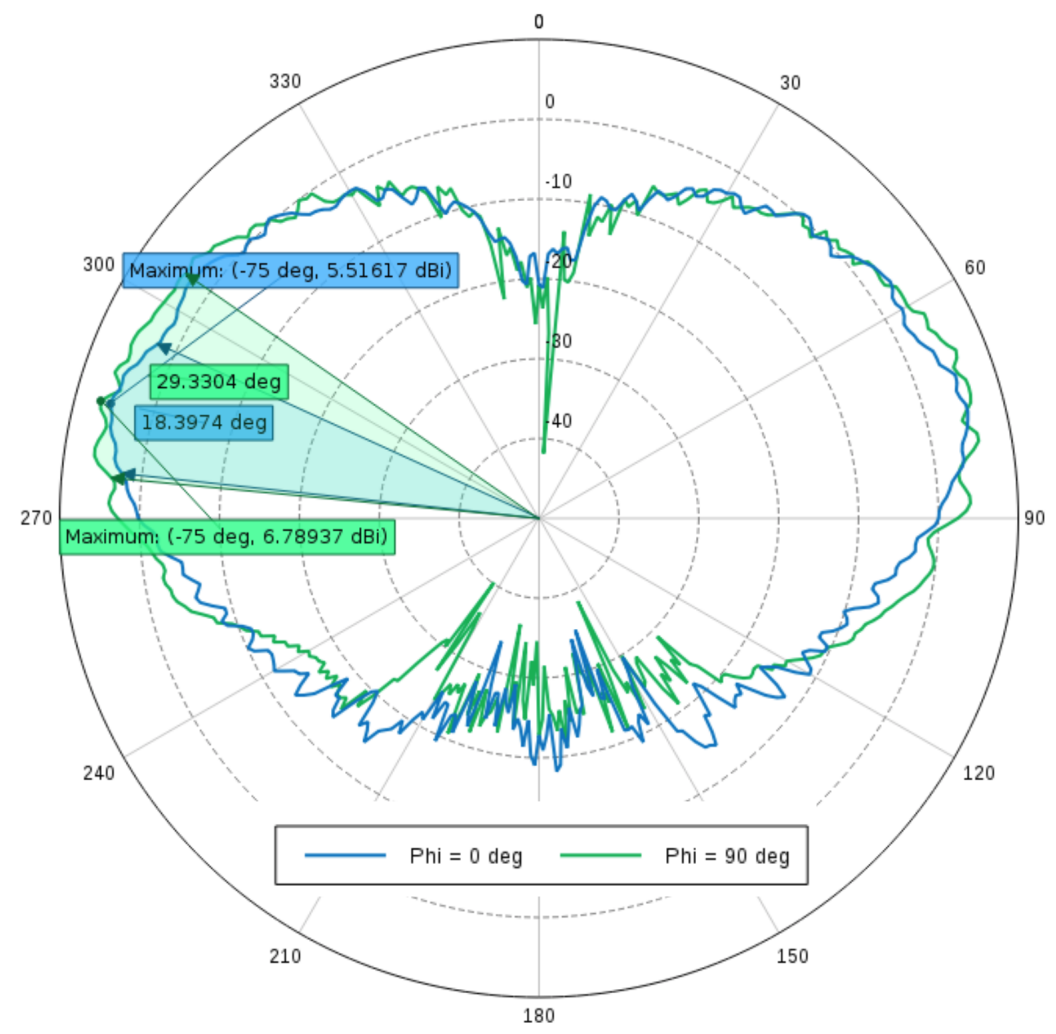

(a) Monopole antenna.

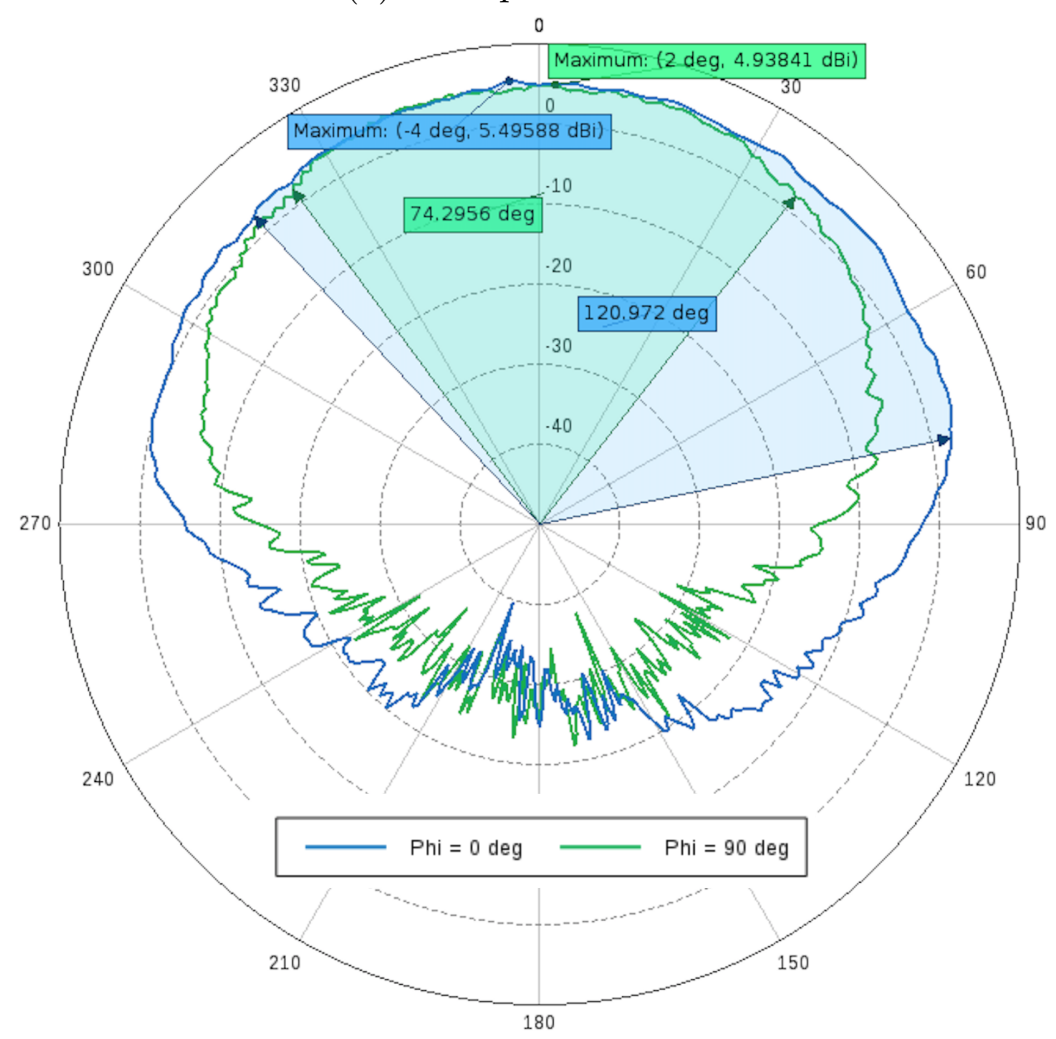

(b) Patch antenna.

Fig. 3.2: Antenna gain on top of a vehicle with side view (blue) and front view (green) at $3.6 \mathrm{GHz}$ in FEKO [28]. 


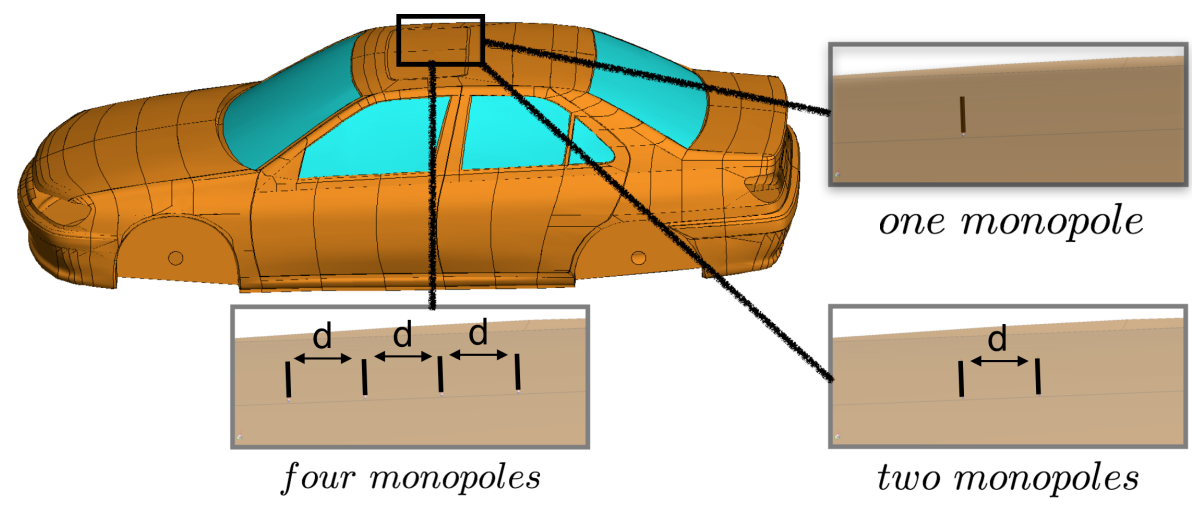

Fig. 3.3: Three different vehicle antenna setups with one, two, and four monopole antennas with an inter-element spacing $d$ between the antennas.

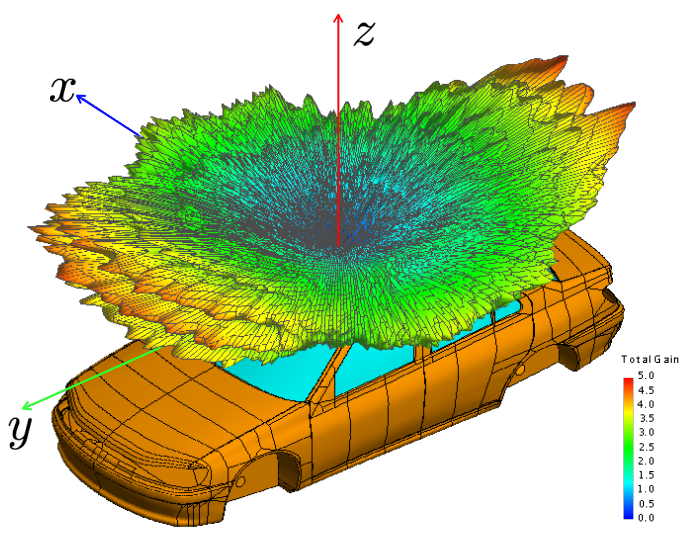

(a) Total gain in linear scale.

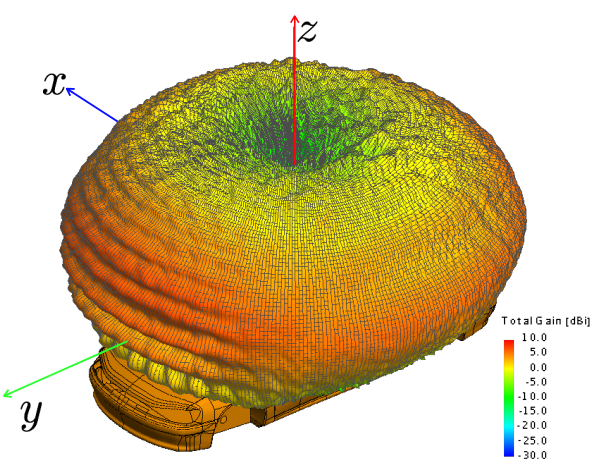

(b) Total gain in logarithmic scale.

Fig. 3.4: Antenna gain of a monopole antenna on top of a vehicle at $3.6 \mathrm{GHz}$ in FEKO [28].

\subsection{Base Station}

Subsequently of determining the antennas on top of the vehicle, the BS configuration is chosen in the following. The BS for conventional MIMO is comprised of one, two or four isotropic antennas for the LOS and NLOS communication. They are chosen to be isotropic in order to transmit towards the receiver positions on the trajectory with the same antenna gain at each position. The isotropic BS makes an averaging of the channel capacity over the entire trajectory with its 1434 positions reasonable for conventional MIMO. Consequently, no beamforming is applied here. The investigation contains a SISO, MIMO 2x2 and 4x4 V2I communication with isotropic antennas at the BS and monopole antennas on top of the car. Table 3.3 gives an overview of the different conventional MIMO configurations for the transmitter (BS) and receiver (vehicle antenna).

From conventional MIMO towards massive MIMO the number of used antennas is increased. The massive BS contains $Q=64$ patch antennas for all massive cases. In the case of massive MIMO, only LOS communication is studied, because the NLOS communication can not be investigated with the current version V14.5 
Table 3.3: Overview of the transmitter and receiver configuration for conventional MIMO.

\begin{tabular}{|l|l|l|}
\hline SISO & Transmitter description & \multicolumn{1}{c|}{ Receiver description } \\
\hline $\begin{array}{l}B S_{1} \text { with one isotropic } \\
\text { antenna (one input) }\end{array}$ & $\begin{array}{l}\text { One monopole antenna on top of } \\
\text { the vehicle (one output) }\end{array}$ \\
\hline MIMO 2x2 & $\begin{array}{l}B S_{2} \text { with two isotropic } \\
\text { antennas (two inputs) }\end{array}$ & $\begin{array}{l}\text { Two monopole antennas on top of } \\
\text { the vehicle (two outputs) }\end{array}$ \\
\hline MIMO 4x4 & $\begin{array}{l}B S_{3} \text { with four isotropic } \\
\text { antenna (four inputs) }\end{array}$ & $\begin{array}{l}\text { Four monopole antennas on top of } \\
\text { the vehicle (four outputs) }\end{array}$ \\
\hline
\end{tabular}

of WinProp [27]. Fig. 3.5 shows three different configurations, which depend on the number of input channels, but keeping the total number elements at the BS constant $(Q=64) . B S_{1}$ is using all antennas to create one beam pointing to the reference points (number of modules $M=1$ ). It is used for the MU-mSISO case. Fig. 3.6 demonstrates the corresponding 3D antenna gain of the $B S_{1}$ pointing towards reference point $P_{1}$ (top) and pointing towards reference point $P_{2}$ (bottom).

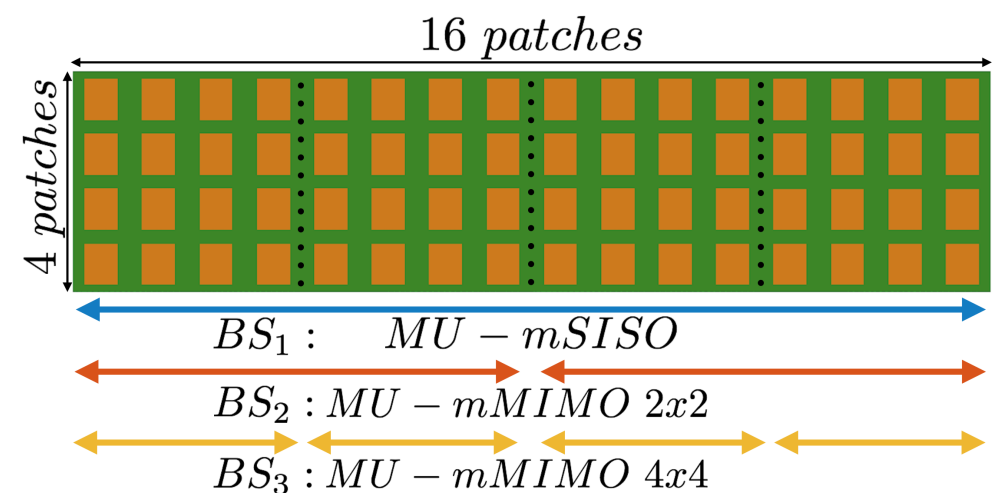

Fig. 3.5: Three different configurations of the BS with 64 patch antennas for massive MIMO communication.

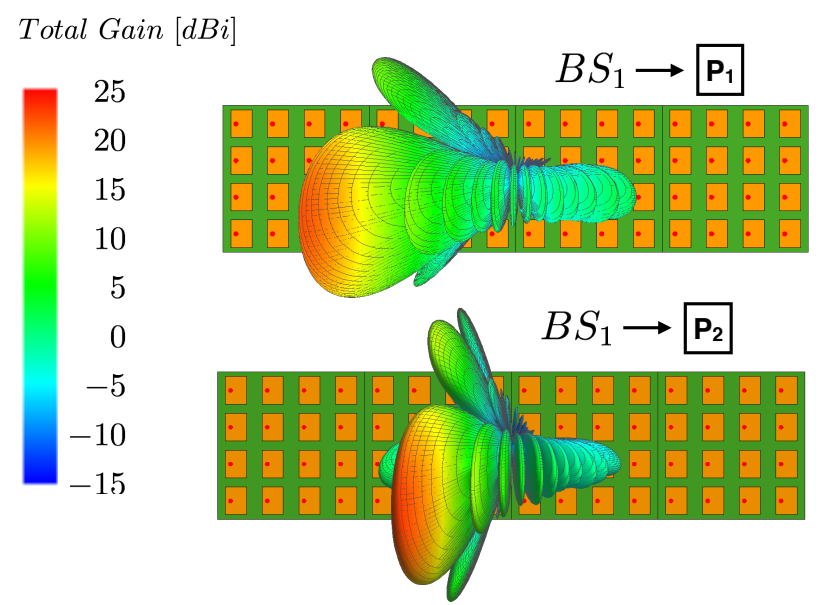

Fig. 3.6: Antenna gain for one massive beam $\left(B S_{1}\right)$ towards reference point $P_{1}$ (top) and towards the second reference point $P_{2}$ (bottom) at $3.6 \mathrm{GHz}$ in FEKO [28].

Table 3.4 gives an overview of the transmitter and receiver for massive MIMO. In 
Table 3.4: Overview of the transmitter and receiver for massive MIMO.

\begin{tabular}{|l|l|l|}
\hline & Transmitter description & \multicolumn{1}{|c|}{ Receiver description } \\
\hline MU-mSISO & $\begin{array}{l}B S_{1} \text { with 64 patch an- } \\
\text { tennas concentrated as } \\
\text { beam (one input) }\end{array}$ & $\begin{array}{l}\text { One monopole antenna on } \\
\text { top of the vehicle (one out- } \\
\text { put) }\end{array}$ \\
\hline MU-mMIMO 2x2 & $\begin{array}{l}B S_{2} \text { with 64 patch an- } \\
\text { tennas working as two } \\
\text { beams (two inputs) }\end{array}$ & $\begin{array}{l}\text { Two monopole antenna on } \\
\text { top of the vehicle (two out- } \\
\text { puts) }\end{array}$ \\
\hline MU-mMIMO 4x4 & $\begin{array}{l}B S_{3} \text { with 64 patch an- } \\
\text { tennas working as four } \\
\text { beams (four inputs) }\end{array}$ & $\begin{array}{l}\text { Four monopole antenna on } \\
\text { top of the vehicle (four out- } \\
\text { puts) }\end{array}$ \\
\hline
\end{tabular}

Table 3.5: Overview of used overall transmitted power.

\begin{tabular}{|l|l|c|}
\cline { 2 - 3 } \multicolumn{1}{c|}{} & Conventional MIMO & Massive MIMO \\
\hline LOS & $10 \mathrm{dBm}$ & $0 \mathrm{dBm}$ \\
\hline NLOS & $30 \mathrm{dBm}$ & \multicolumn{1}{c|}{-} \\
\hline
\end{tabular}

order to steer the beam towards a certain position, a proper coefficient and phase need to be set for each element. The phases are calculated in advance for each of the three positions of the car. They are calculated as explained in Section 2.2.2 with the previous knowledge of the specific position of the vehicle, while the coefficients are selected to be uniform. The transmitted power is always equally distributed over all channels; hence no water filling is used. In order to get reasonable SNR a realistic power needs to be transmitted. For automotive MIMO applications a SNR between $10-20 \mathrm{~dB}$ et al. [29] is reasonable. Table 3.5 depicts the used overall transmitted power for conventional and massive MIMO in LOS and NLOS. Therefore, the transmitted power for LOS and NLOS is selected to be $10 \mathrm{dBm}$ and $30 \mathrm{dBm}$, respectively.

Fig. 3.7 illustrates the corresponding power map for the MU-mSISO case at the height of the car rooftop (1.42 meter), when the BS is transmitting to $P_{1}$ (see Fig. 3.7a) and towards $P_{2}$ (see Fig. 3.7b). Here it can be seen that the power is concentrated towards the reference point; hence the inference is reduced for other users which are not too close to the similar reference point.

Then, $B S_{2}$ is subdivided in two modules of $4 \times 8(M=2)$, reducing the focusing capabilities of each channel to only 32 elements. It is used for MU-mMIMO $2 \times 2$. Finally, the third configuration, $B S_{3}$ is composed of four $4 \mathrm{x} 4$ antenna arrays $(M=$ 4). Four beams are created with a wider width and they are used for MU-mMIMO $4 \times 4$. 


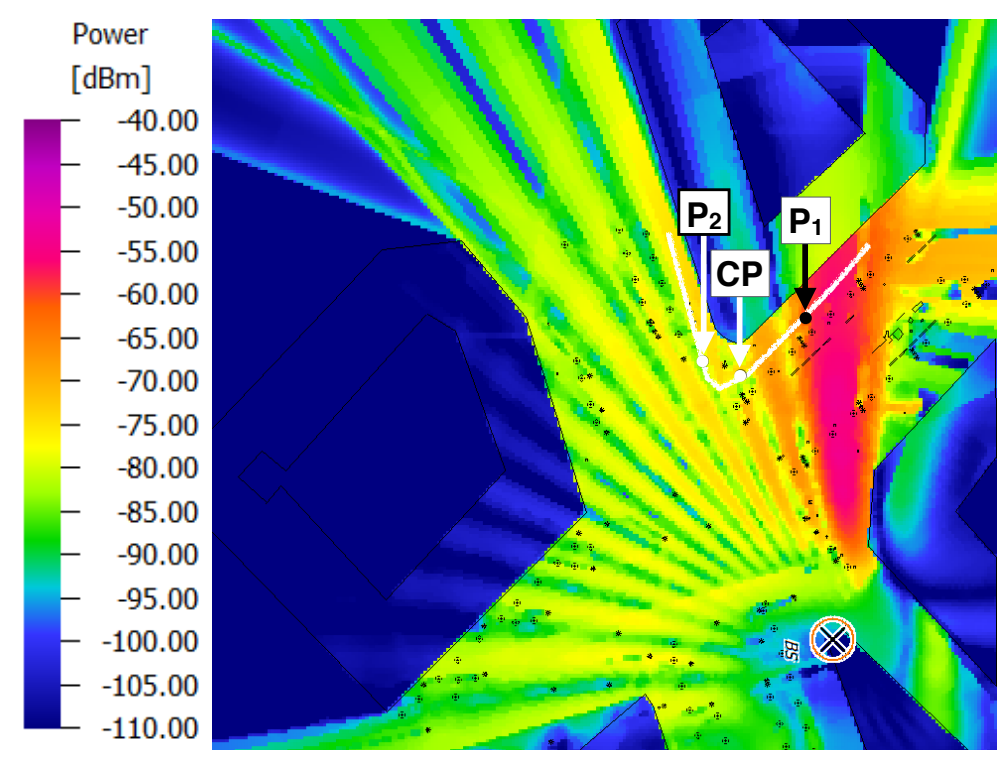

(a) MU-mSISO beam $\left(B S_{1}\right)$ pointing towards reference point $P_{1}$.

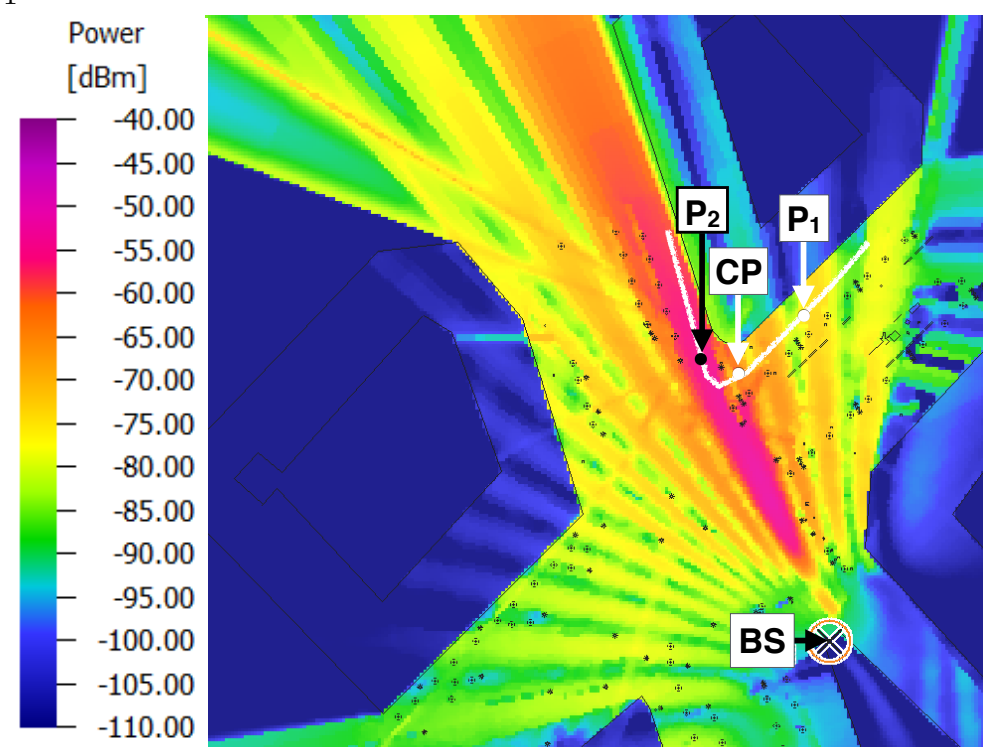

(b) MU-mSISO beam $\left(B S_{1}\right)$ pointing towards reference point $P_{2}$.

Fig. 3.7: Power map of the incoherent power received at a height of 1.42 meters and with a resolution of 1 meter in WinProp [27].

\subsubsection{Validation of Propagation Environment}

Taking the impact of all the objects in the scenario into account, specially the ground, can be critical in the final distribution of the fields [30]. The propagation software, by means of the ray tracing method, considers the interaction of several points in the environment between the transmitter location and the receiver points considered in the reference trajectory. In order to validate whether it is consistent or not with the study in [30], a straight line in LOS condition for all points is assumed, starting at the BS coordinates, but at the same height as the car. The target, which is in this case reference point $P_{2}$, is also included in the new set of points. Fig. 3.8 shows on the right side the trajectory of the vehicle. The vehicle starts below the BS 


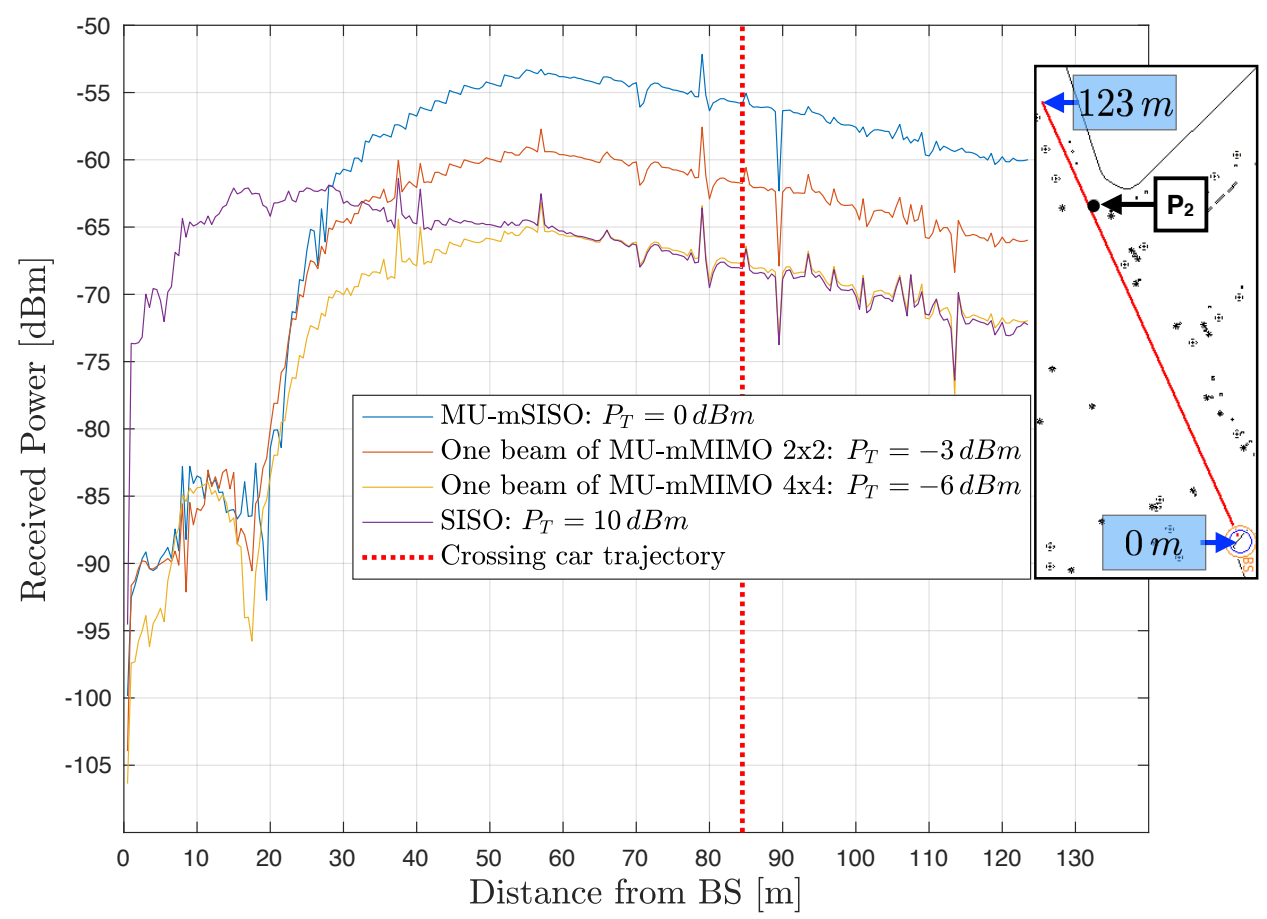

Fig. 3.8: Received power for massive MIMO towards $P_{2}$ and conventional SISO as a function of distance at a height of $1.42 \mathrm{~m}$.

at 0 meter, it crosses the reference point $P_{2}$ and stops at a distance of 123 meter away from the BS. As can be seen in Fig. 3.8, the received power after adding coherently the contribution of the multi-path depends on the configuration used in each case. The SISO model, in which a single patch antenna is used at the transmitter, follows the expected tendency. The maximum of received power is found some meters after the transmitter and from this point on the function decreases monotonically with a constant slope of $27 \mathrm{~dB} /$ decade, which agrees with [30]. On the other hand, when the massive BS is introduced, an important difference may be noticed. The maximum is located at a further distance. As it was expected, the beam focusing on the user concentrates the power on a region close to the vehicle. If all elements are used as a single beamformer, which is the case of MU-mSISO, the maximum power is larger than in the case of conventional SISO. This behavior denotes the higher efficiency of this type of systems, which are able to deliver more power to the user even with lower consumption, as it is the case here. For MU-mMIMO, the behavior is very similar but the usage of $1 / M$ of the total power at each module reduces the received power.

Due to the use of uniform weighting amplitudes, the presence of a sidelobe produces a small peak in the first meters. The level of the sidelobe depends on the number of antennas per module, thus this spurious peak is larger as the order of the MIMO increases (less elements to create the beam). In general, the three massive configurations show the same behavior, with different power levels according to their modular distribution. 


\section{Chapter 4}

\section{Methodology}

The following chapter describes the methodology used in this work. The preamble gives a general overview of the main steps and tools used in order to obtain plausible conclusions. This is followed by the description of how the data is collected. Then, the exploit methods of numerical analysis are specified. Finally the data analysis and the corresponding figure of merit are explained.

\subsection{Preamble}

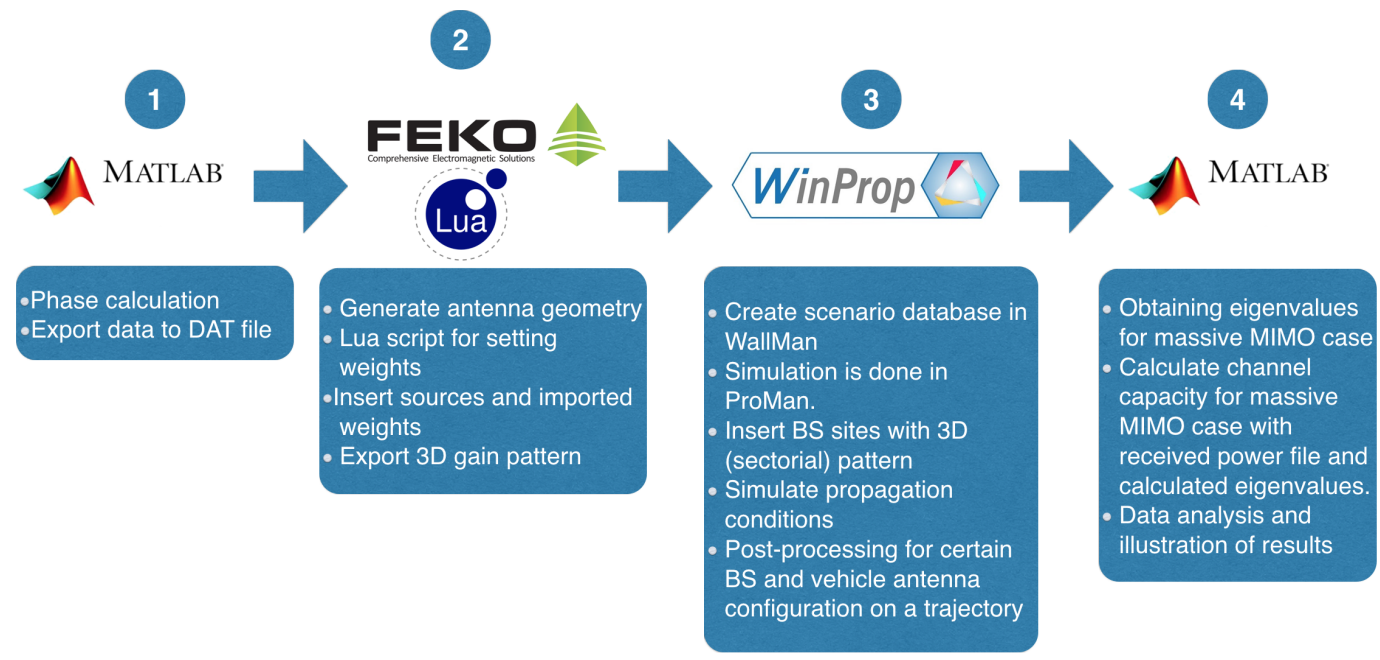

Fig. 4.1: Overview of the procedure, its four steps, and the used programs for the numerical V2I communications research model.

Fig. 4.1 presents an overview of the four main steps to this numerical V2I communication research model. First, $M A T L A B$ is used for the calculation of the phases of the massive MIMO BSs as explained in Section 2.2.2. These phases are exported as data files. Then, FEKO, an electromagnetic simulation software, is used to design the different antennas. Furthermore, a $L u a^{1}$ script is needed in order to set the 64 weights for the massive MIMO BSs. For the massive MIMO antenna gain pattern the uniform coefficients and pre-calculated phases are considered. After

\footnotetext{
${ }^{1}$ Lua is a scripting language [31] which is compliant with FEKO.
} 
setting the antenna weights with the Lua-script, the distinct antenna gains are obtained. WinProp consists of WallMan for creating the database and ProMan for the propagation channel simulation, among other tools which are not considered in this study. For simplification ProMan is called WinProp from now on. In step three, WinProp simulates the propagation environment using the scenario database created in advance with WallMan, i.e., a portion of the city of Barcelona. It offers an accelerated post-processing where the distinguishable antenna gains are inserted. Finally, MATLAB obtains the eigenvalues from the channel condition numbers and calculates together with the received power files the channel capacity in the massive MIMO case. Moreover MATLAB is used for the illustration of all results.

\subsection{Method}

The data used for the analysis, i.e., the channel capacity, is obtained in step three (see Fig. 4.1) for conventional MIMO by WinProp for each point on the trajectory (1434 points). The channel capacity is calculated each decimeter for the still position of the vehicle antenna configuration. With fixed transmitted power, a different SNR and, consequently, channel capacity is received, through varying multi-path situations. The channel matrix is calculated for the central point at the receiver position. Then, the MIMO channel matrix is determined by the phase shifts between the single array elements. The MIMO post-processing tool (in WinProp) is approximating the MIMO channel matrix by adding a phase shift to angles of departure and arrival depending on the inter-element spacing. For more details about the MIMO postprocessing tool, the reader is encouraged to refer to [27].

In the case of massive MIMO the data is collected in a different way. The reason being that WinProp version 14.5. is not able to calculate channel capacity for the significantly higher received power. The program still provides the correct received power. Henceforth, the channel capacity has to be calculated in MATLAB by using the eigenvalues and the coherent received power. The eigenvalues are not given either by the simulation software, instead the channel condition number from the Frobenius normalized channel matrix (see (2.8)) is provided. The property of the eigenvalues obtained from the Frobenius normalized channel matrix is, that the sum is equal to one. The channel condition number for two, three, and four number of modules, assuming an additive behavior of the eigenvalues in all cases, can be expressed as:

$$
\begin{aligned}
& C N_{1}=\frac{\lambda_{1}}{\lambda_{2}} \\
& C N_{2}=\frac{\lambda_{1}}{\lambda_{3}} \\
& C N_{3}=\frac{\lambda_{1}}{\lambda_{4}}
\end{aligned}
$$

Therefore, the eigenvalues for the massive MIMO 2x2 case can be obtained by the following equation:

$$
\begin{array}{r}
1=\lambda_{1}+\lambda_{2} \\
1=\lambda_{1}+\lambda_{1} \cdot C N_{1} \\
\lambda_{2}=\lambda_{1} \cdot C N_{1}
\end{array}
$$


For massive MIMO 4x4 the equation system is stated as:

$$
\begin{array}{r}
1=\lambda_{1}+\lambda_{2}+\lambda_{3}+\lambda_{4} \\
1=\lambda_{1}+\lambda_{1} \cdot C N_{1}+\lambda_{1} \cdot C N_{2}+\lambda_{1} \cdot C N_{3} \\
\lambda_{2}=\lambda_{1} \cdot C N_{1} \\
\lambda_{3}=\lambda_{1} \cdot C N_{2} \\
\lambda_{4}=\lambda_{1} \cdot C N_{3}
\end{array}
$$

The equation system (4.2a) and (4.3a) are solved by calculating for each the one unknown variable $\lambda_{1}$ in $(4.2 \mathrm{~b})$ and (4.3b), respectively.

\subsection{Numerical Analysis}

In order to accomplish a sophisticated model of a V2I communication, appropriate data analysis tools need to be selected. The communication model can be separated into electromagnetic modeling of the antennas and the wave propagation modeling between the BS and the vehicle. As already explainted the company Altair offers software to simulate different kind of technical challenges in various engineering areas, i.a., FEKO, and WinProp.

The software suite FEKO provides different solvers to approximate electromagnetic fields. The Maxwell's equations might be approximated apart from the time intensive Method of Moments (MoM) with the Multilevel Fast Multipole Method (MLFMM), which is an alternative conceptualization of the MoM. "It was shown that the MLFMM can solve electromagnetically large complex problems using only a fraction of the memory and CPU-time required by the full MoM. The errors introduced are fully controllable and this enables the MLFMM to produce accurate results." [32] Due to this benefit MLFMM is selected as the solver to obtain the far field antenna gain pattern for the monopole antennas on top of the vehicle and for different BSs.

WinProp utilizes ray tracing with GTD and UTD for the calculation of the diffraction coefficients and the Fresnel's equations for reflections coefficients as explained in Section 2.1. With importing the radiation pattern of the antenna gain previously calculated with $F E K O$, it is possible to obtain the channel matrix for a particular scenario.

\subsection{Data Analysis}

After elaborating the numerical analysis, i.e. the methods the simulation tools use, a figure of merit is specified. Furthermore, it has to be presented in a detailed account of how the data is actually analyzed.

Based on the obtained channel capacities, the comparison of the different MIMO configurations, i.e., SISO, MIMO 2x2 and MIMO 4x4 is done by studying the figures of merit which are the arithmetic average over all 1434 obtained channel capacities for each MIMO configurations on the trajectory:

$$
C_{a v .}=\frac{1}{W} \sum_{i=1}^{W} C_{i}
$$


$W$ is the number of receiver positions, i.e. $W=1434$. The noise power at the receiver is fixed to a level of $P_{N}=-90 \mathrm{dBm}$ for all positions on the trajectory of the vehicle. In LOS the transmitted power is $P_{T}=10 \mathrm{dBm}$ for the conventional configurations and in NLOS the transmitted power is $P_{T}=30 \mathrm{dBm}$. Thus for each case three average channel capacities are obtained and can be compared.

The figure of merit for the massive MIMO for LOS is also obtained by the average channel capacity (4.4) with the difference that the number of receiver positions is selected to be $W=300$ for all massive MIMO configurations i.e. MU-mSISO, MUmMIMO $2 \times 2$ and MU-mMIMO 4x4. The half power beamwidth of the massive BS is varying for each configuration. Even so $W=300$ is chosen to be the same of all massive configurations; hence illuminated region is as summed to be the same. The receiver noise level is $P_{N}=-90 \mathrm{dBm}$ as for the conventional MIMO case. The overall transmitted power is chosen to be $P_{T}=0 \mathrm{dBm}$.

For the investigation of different inter-element spacing for conventional MIMO the mean SNR over the entire trajectory is chosen to be $10 \mathrm{~dB}$. If the mean SNR method is used the transmitted power and receiver noise level is chosen to be so that the SNR is on average the mean SNR. Moreover, the average channel capacity $C_{a v}$ is calculated over the whole trajectory $(W=1434)$. The inter-element spacing is investigated starting by 0.1 wavelength up to 4 times the wavelength $(0.83-33.2 \mathrm{~cm})$ in 0.1 wavelength steps. 


\section{Chapter 5}

\section{Simulation Results and Analysis}

The next chapter presents, analyses and discuses the simulation results of the modeled V2I communication in a intersection of two streets in Barcelona. The following MIMO modalities are investigated: conventional MIMO, massive MIMO and conventional MIMO with different inter-element spacing.

\subsection{Conventional MIMO}

In the case of conventional MIMO the BS is transmitting with an overall power of $10 \mathrm{dBm}$ and $30 \mathrm{dBm}$ for two BS positions, LOS and NLOS, respectively. The receiver noise level is selected to be $P_{N}=-90 \mathrm{dBm}$ for all cases. Fig. 3.1b shows the scenario with the exact locations of the BS and the trajectory of the vehicle.

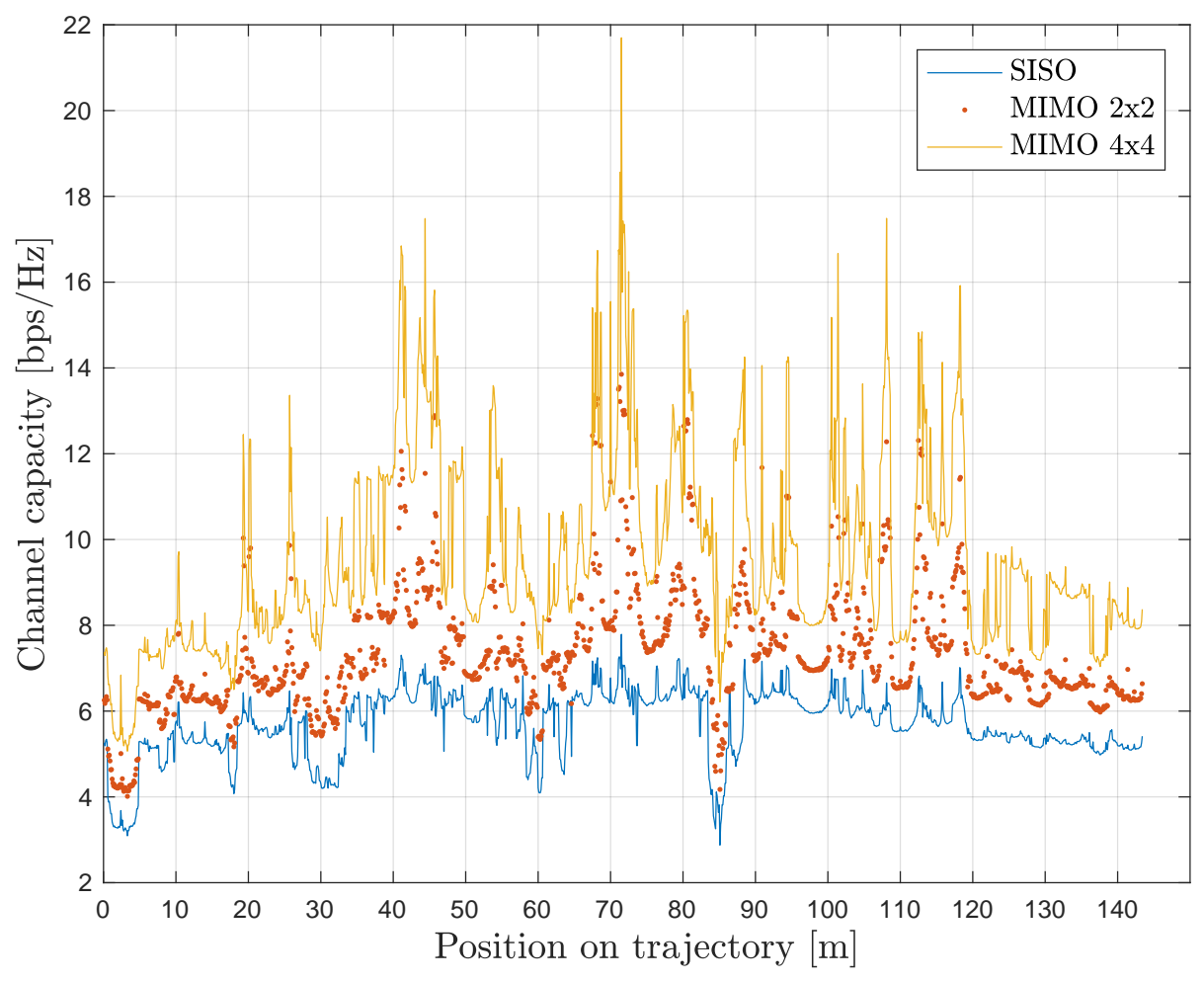

Fig. 5.1: Channel capacity for conventional MIMO in LOS. 
First the results for the LOS communication are presented and discussed. Fig. 5.1 shows the obtained channel capacity at each position for different conventional MIMO configurations with a step size of the vehicle of each decimeter on the trajectory. Comparing SISO and MIMO 2x2, the latter provides a gain in capacity for all positions. This improvement in terms of channel capacity is obtained due to the so called MIMO gain which depends on the richness of the channel. Between SISO and MIMO 4x4 an even higher channel capacity is received. Here, contrastive the gain is not significant better for all positions using MIMO 4x4 instead of MIMO 2x2, even the channel capacity is greater for the majority of the receiver locations. Only for channels rich enough in terms of scattering, the associated capacity would noticeably increase with the MIMO order. Another way of looking at it is in terms of the rank of the channel matrix $\mathbf{H}$, where the rank four is not always assured on the trajectory. It can be observed that the channel capacity for SISO, MIMO 2x2, and $4 \mathrm{x} 4$ is highest approximately at 70 meters ( $C P$ point) on the trajectory. This maximum is reached due to the shortest distance between the BS location and vehicle on the trajectory. Furthermore, Fig. 5.2 illustrates the average channel capacities

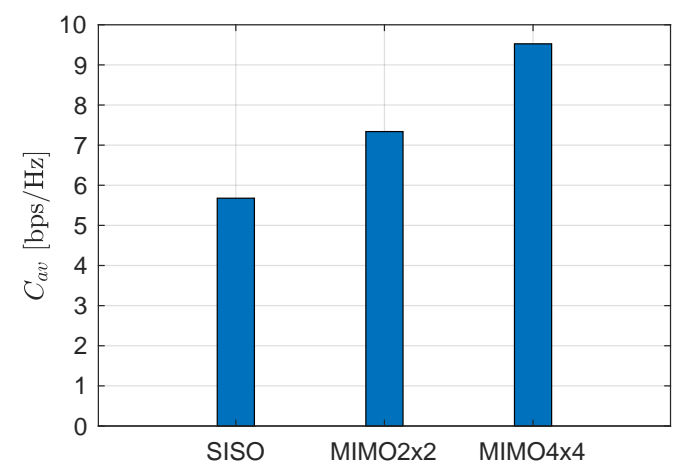

(a) Average channel capacity.

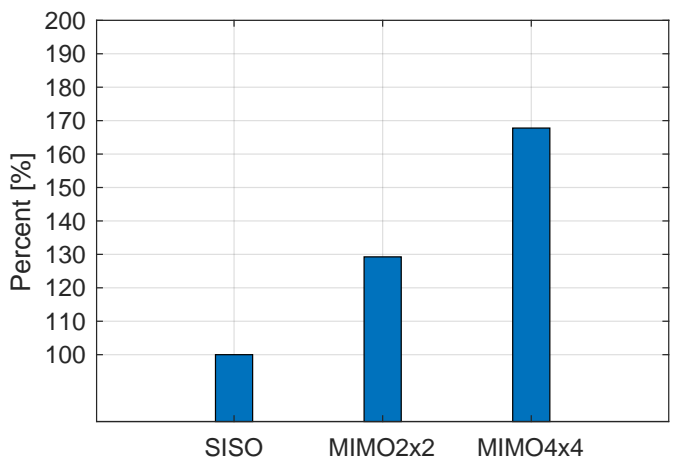

(b) Average capacity in percentage respect to SISO.

Fig. 5.2: Average channel capacity for conventional MIMO over the entire trajectory (mean SNR of $13 \mathrm{~dB}$ ) and LOS.

for LOS. Fig. 5.2a shows the capacity in bits per seconds per hertz and Fig. 5.2b presents it in percentage with respect to the SISO case. It can be concluded that even so the channel is not rank four, the average channel capacity is highest for MIMO 4x4. Fig. 5.2b evinces $130 \%$ and $170 \%$ more capacity in average going from SISO to MIMO 2x2, and $4 \mathrm{x} 4$, respectively. 


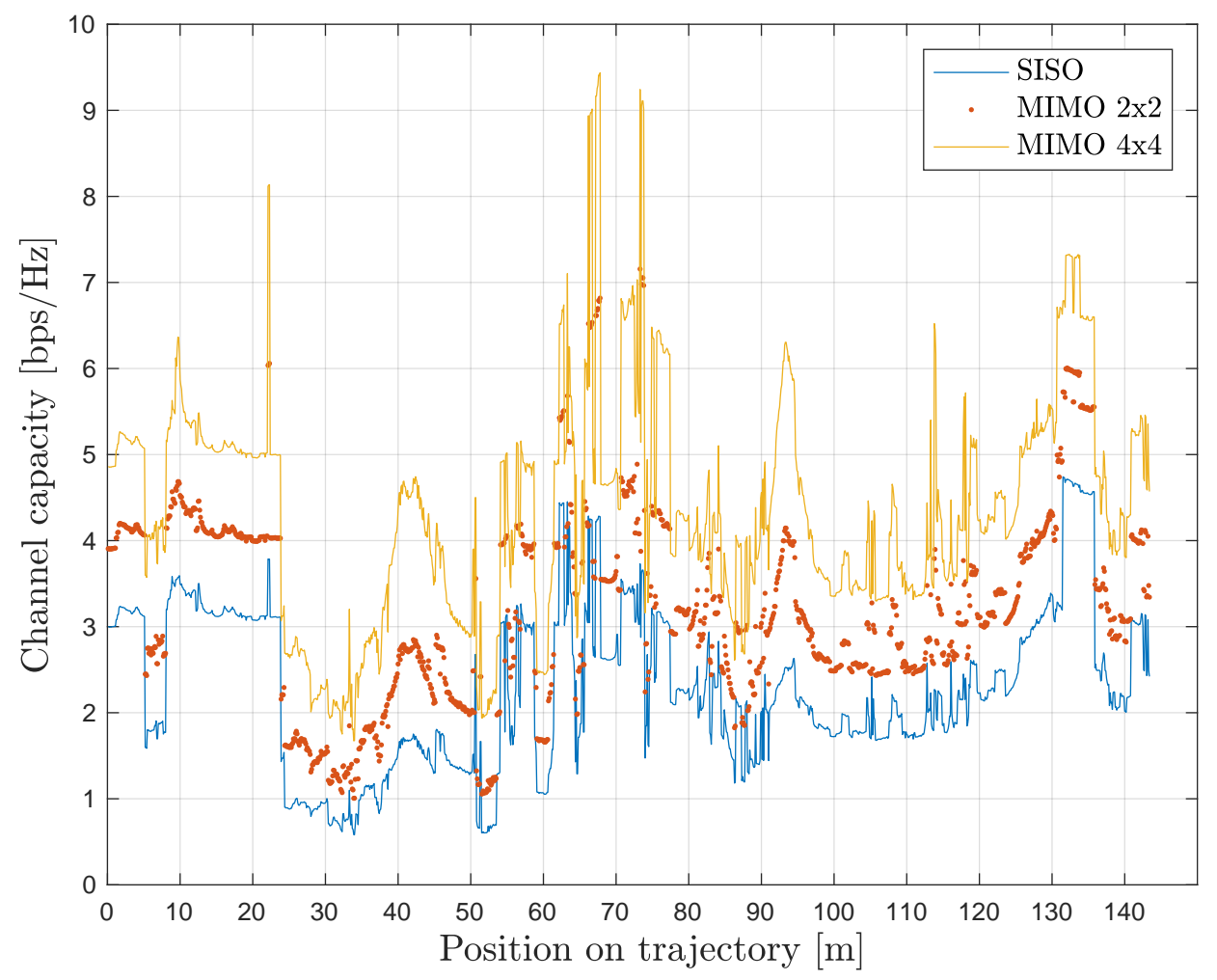

Fig. 5.3: Channel capacity for conventional MIMO in NLOS.

For the NLOS communication the transmitted power is increased to $30 \mathrm{dBm}$ due to the larger distance (see Fig. 3.1b position of $B S_{C}^{N L O S}$ ) and higher propagation losses. Fig. 5.3 depicts the obtained channel capacity for the NLOS communication of the conventional MIMO configurations. The highest channel capacity is available around 70 meters ( $C P$ point) as in the LOS case, where the distance between vehicle and BS is the minimum. Furthermore, the evolution of the channel capacity for the different configurations is behaving in the same way. This means that if the SISO capacity drops the MIMO 2x2 and MIMO 4x4 is also reduced, e.g. at 23 meter. It can be inferred that this behavior is given by the shadowing of certain propagation paths and therefore less received power. Particularly, at 23 meter shadowing eminently can be observed because also the SISO channel capacity drops drastically at 23 meter, but also at other positions (e.g. at 136 meter).

The ratio of the channel capacity between the three configurations is almost constant. This indicates that the channel is rich in terms of scatterer and the channel condition number is small. The smaller the channel condition number becomes the richer is the channel and consequently the higher is the MIMO gain. Fig. 5.4a presents a comparison of the average channel capacity for conventional MIMO in NLOS first in bits per second per hertz. Fig. 5.4b shows the channel capacity in percent with respect to the SISO case. By increasing the order of the configuration from SISO towards MIMO 2x2 $140 \%$ more and for MIMO 4x4 $190 \%$ more average channel capacity is achieved.

A comparison between LOS and NLOS should only be done by comparing the percentage gain with respect to each SISO case. Fig. 5.4b shows this gain for NLOS 
and Fig. 5.2b for LOS. For MIMO 2x2 $10 \%$ more channel capacity is gained for NLOS communication with respect to LOS. A remarkable increase of $20 \%$ is obtain for MIMO $4 \mathrm{x} 4$ in NLOS with repect to the LOS due to the richness of the NLOS communication channel.

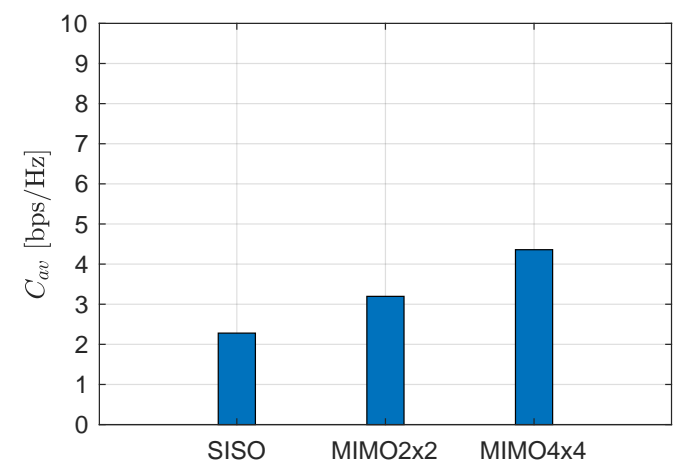

(a) LOS

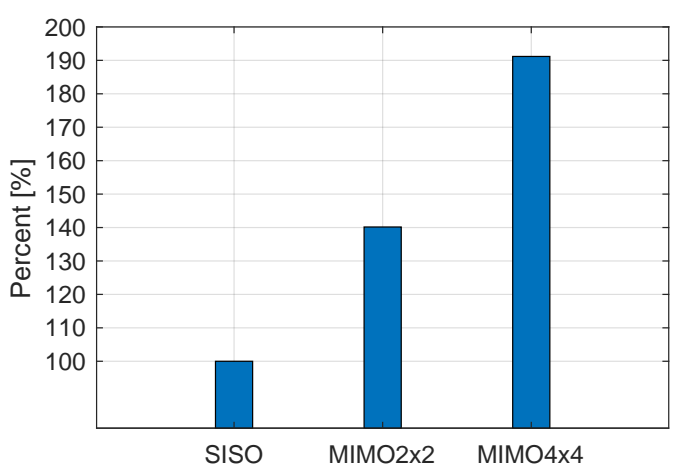

(b) Average capacity in percentage respect to SISO.

Fig. 5.4: Average channel capacity for conventional MIMO over the entire trajectory (mean SNR of $8 \mathrm{~dB}$ ) and NLOS.

\subsection{Massive MIMO}

After investigating the conventional MIMO with single antenna elements, massive MIMO with 64 patch antennas at the BS is examined here. Two distinct vehicle positions are studied, i.e., $P_{1}$ and $P_{2}$. The overall transmit power is $0 \mathrm{dBm}$ for the observed LOS case. This reduction of transmit power with respect to conventional MIMO is compensated by beamforming, which concentrates the transmit power towards the reference points. The channel capacity is calculated with the received coherent power and the obtained eigenvalues. Using (2.5) the channel capacity is calculated, where the receive noise power is selected to be $P_{N}=-90 \mathrm{dBm}$.

When the received power is considered, the trajectory may be divided in two regions. First, the illuminated region corresponds to the main beam footprint, where the user vehicle is located and received power should be higher. The second can be called as shadow region and lies outside of the illuminated area, where the radiated 


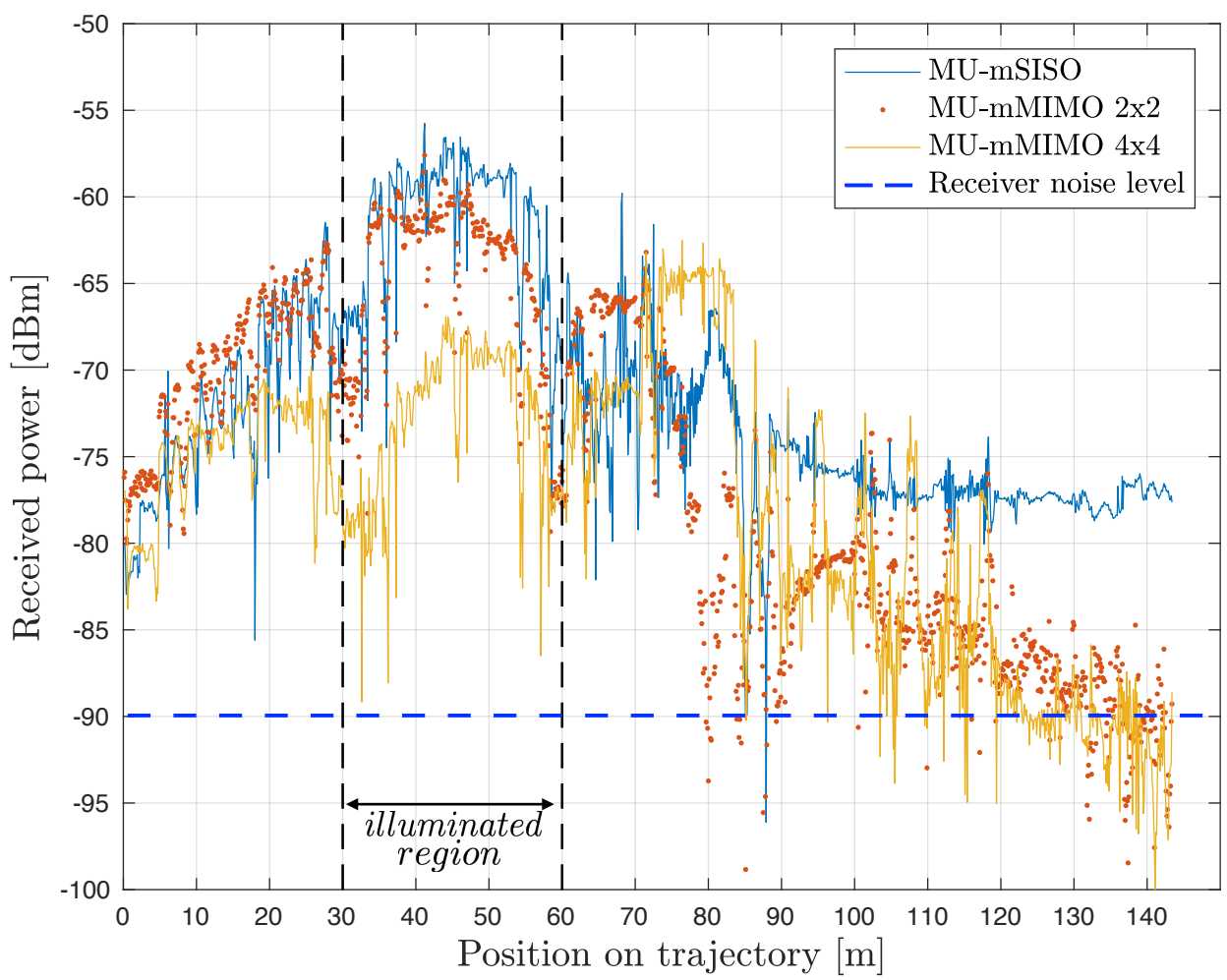

Fig. 5.5: Received power for massive MIMO towards reference point $P_{1}$ at each position on the trajectory.

power should be as small as possible to reduce the inter-user interference and improve the efficiency. The illuminated region has been defined as a 30-meter interval centered on the point where the BS is pointing at. In the case of beamforming towards $P_{1}$, the area where the user is expected lies between 30 and $60 \mathrm{~m}$ and, for the case of beamforming towards $P_{2}$, it is located between 70 and $100 \mathrm{~m}$.

First, the coherent received power is studied for two positions of the car. In the case of beamforming towards $P_{1}$, the illuminated region lies between 30 and 60 meter and for a beamforming towards $P_{2}$ the illuminated region lies between 70 and 100 meter. Fig. 5.5 shows the received coherent power for MU-mSISO, MU-mMIMO 2x2, and MU-mMIMO 4x4 in blue, red, and yellow, respectively. The illuminated region is denoted by the vertical dashed line. It is shown that between 30 and 60 meter the received power is highest for MU-mSISO, smaller for MU-mMIMO 2x2 and smallest for MU-mMIMO 4x4. This is due to constructive superposition of the two MU-mMIMO 2x2, and four MU-mMIMO 4x4 superimposed beams. The overall interference is smallest for other users for MU-mMIMO 4x4, than MU-mMIMO 2x2 and biggest MU-mSISO. This interference is obtained by integrating the coherent power over the shadow region. Moreover, the footprint of the BS is getting broader from MU-mSISO to MU-mMIMO 4x4. With respect to MU-mMIMO $4 \mathrm{x} 4$ the maximum received power is close to $C P$ point (around $70 \mathrm{~m}$ ) even if $P_{1}$ lays not in this area. Causes for this behavior may lay in the distance between BS and vehicle, which is the shortest at 70-80 meter and destructive superposition of coherent power at the illuminated region (between 30 and 60 meter).

Fig. 5.6 depicts the received power for massive MIMO towards $P_{2}$. This point lies after the corner of the intersection (see Fig. 3.1b) and is henceforth diverging from 


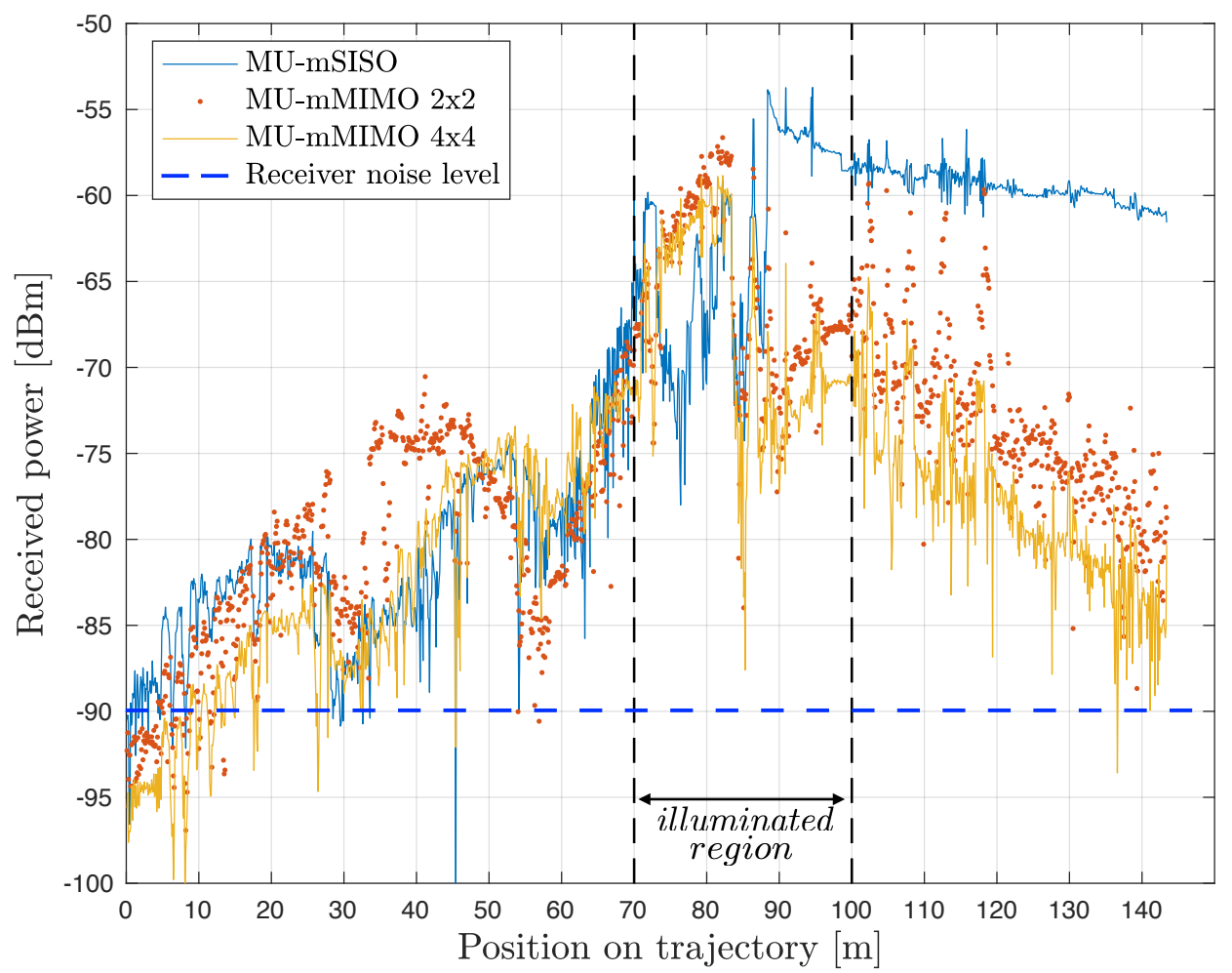

Fig. 5.6: Received power for massive MIMO towards reference point $P_{2}$ at each position on the trajectory.

$P_{1}$. The analysis here is more challenging as the power is fading stronger than with the concentrated beams towards $P_{1}$. Fig. 5.6 shows at 85 meter for MU-mMIMO 4x4 a step of $25 \mathrm{~dB}$, whereas Fig. 5.5 illustrates at 38 meters a step of $15 \mathrm{~dB}$ for MUmMIMO $4 \times 4$ towards $P_{1}$. This indicates a strong cancellation due to superposition of multi-paths, also known as fading. Furthermore, received power is just reduced by $3 \mathrm{~dB}$ from 100 meter until 143 meter for MU-mSISO. The reasoning therefore is that the BS is comprised of four patch antennas in the vertical axis and henceforth the resolution and focusing capability is significantly smaller than in the horizontal axis, where the BS consists of 16 patch antennas. It can be concluded that massive MIMO reduces the interference for other users in comparison to conventional MIMO significantly. It seems to get reduced the most with MU-mMIMO 4x4.

Secondly, the eigenvalues are analyzed for reference point $P_{1}$ and $P_{2}$. The analysis of the eigenvalue of the LOS communication is an challenging task. In order to understand the obtained eigenvalues at a specific position, the simulation software, i.e., WinProp offers a tool that allows to show all arriving rays at a certain point on the trajectory. In general the ideal eigenvalues are uniformly distributed for the purpose of obtaining the maximum channel capacity. In a very rich scenario in terms of multi-path, values close to the ideal uniform distribution are expected $\left(\lambda_{i}=\right.$ $1 / N$ for MIMO $M x N, i=1, \ldots, N)$. Fig. 5.7a depicts the Frobenius normalized eigenvalues for MU-mMIMO $2 \times 2 \lambda_{1}$ in blue and $\lambda_{2}$ in red. Fig. 5.7b shows the eigenvalues for MU-mMIMO $4 \mathrm{x} 4$ with $\lambda_{1}, \lambda_{2}, \lambda_{3}$ and $\lambda_{4}$ in blue, red, yellow and purple, respectively. In both cases the beams are focused towards $P_{1}$. Fig. 5.7a shows almost ideal uniformly distributed eigenvalues for MU-mMIMO 2x2 between 40 and 48 meters on the trajectory. In the same region the eigenvalue $\lambda_{3}$ is strong with 


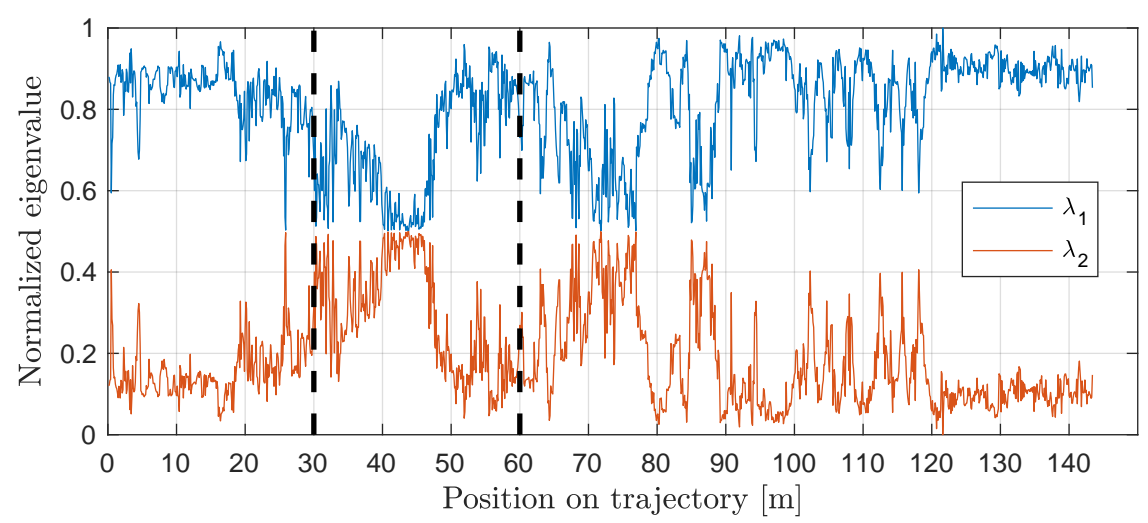

(a) Eigenvalues MU-mMIMO 2x2.

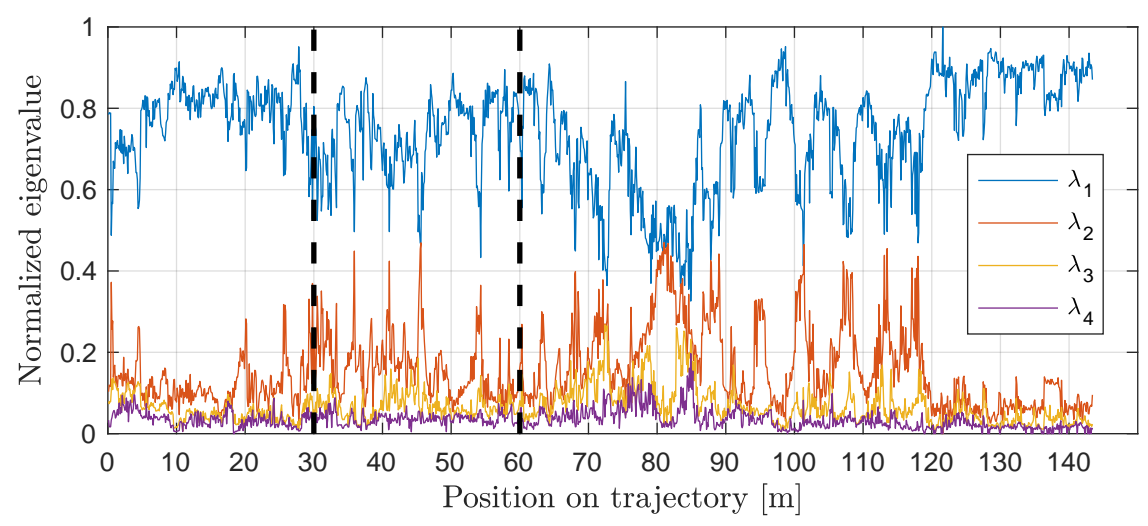

(b) Eigenvalues MU-mMIMO 4x4.

Fig. 5.7: Frobenius normalized eigenvalues for MU-mMIMO 2x2 and 4x4 at each position on the trajectory with beamforming towards $P_{1}$.

a magnitude of approximately 0.1 . Whereas $\lambda_{4}$ is almost in the whole illuminated region around 0.05 for the case of $4 \times 4$.

Fig. 5.8 shows the Frobenius normalized eigenvalues for the massive beams of MU-mMIMO 2x2 and MU-mMIMO $4 \times 4$ towards $P_{2}$. Here, the channel is more heterogeneous for MIMO $4 \mathrm{x} 4$ as in the case of massive MIMO towards $P_{1}$ (see Fig. 5.7). In the case of steering the beam towards $P_{2}, \lambda_{3}$ and $\lambda_{4}$ is higher than in the first case. It is demonstrated that the eigenvalues are still heterogeneous even for the high directive beams. Nevertheless it is necessary to take into account the received power at the illuminated region. The impact of the eigenvalues on the channel capacity is presented in the following paragraph.

Finally, the channel capacity is obtained and investigated for all massive MIMO configurations. Fig. 5.9 shows the massive MIMO channel capacities with focusing towards $P_{1}$. In the illuminated region the channel capacity is highest for MUmMIMO 4x4 followed by MU-mMIMO 2x2 and smallest for MU-mSISO. The maximum of the fourth order massive MIMO around 80 meters is due to the closest distance between the trajectory and BS and therefore the high coherent received power (see Fig. 5.5). Fig. 5.10 depicts the channel capacities for all massive MIMO configurations towards $P_{2}$, where MU-mMIMO $4 \mathrm{x} 4$ is significantly higher from 70 to 85 meters than all others. At 85 meters the channel capacity is reduced even under the level of the MU-mSISO configuration, even so the eigenvalues are almost 


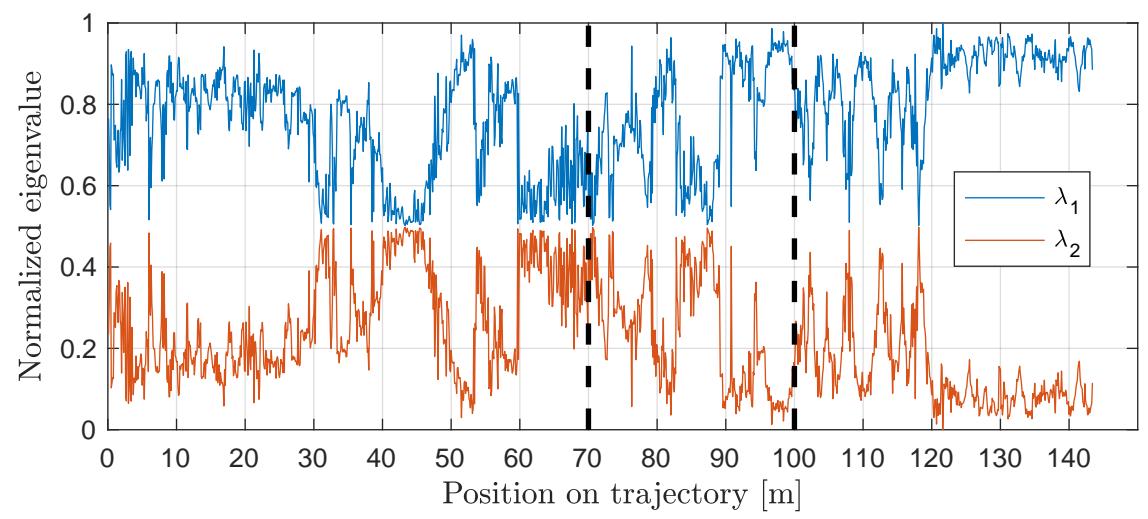

(a) Eigenvalues MU-mMIMO 2x2.

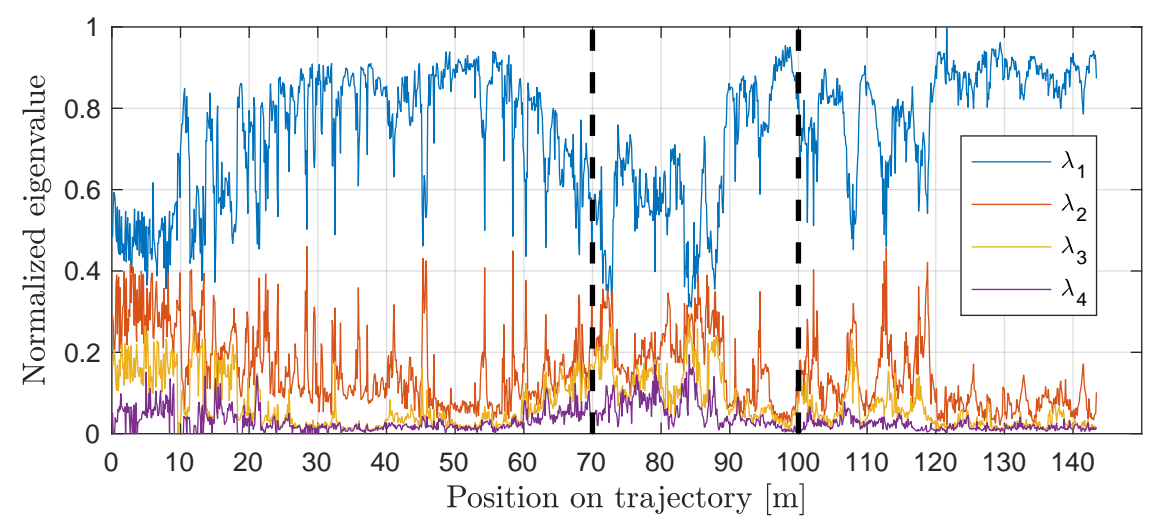

(b) Eigenvalues MU-mMIMO 4x4.

Fig. 5.8: Normalized eigenvalues for MU-mMIMO $2 \times 2$ and $4 \times 4$ at each position on the trajectory with beamforming towards $P_{2}$.

uniformly distributed at the point (see Fig. 5.8 at 85 meter). Fig. 5.6 shows a strong fading of the coherent received power at 85 meter and is the reason for the drop of capacity. Fig. 5.10 shows that after the drop of capacity at 85 meter, the capacity is highest for MU-mMIMO 4x4 until 100 meter, followed by MU-mMIMO 2x2 even it is smaller than MU-mSISO at same points. It is noticeable that for MU-mMIMO second and fourth order the channel capacity is increased with higher received power but without losing the heterogeneity of the eigenvalues.

Fig. 5.11 gives an overview of the average channel capacities of all massive MIMO configuration for beamforming towards $P_{1}$ and $P_{2}$. Fig. 5.12a shows the average channel capacity and Fig. 5.12b illustrates the corresponding average SNR. Fig. 5.11c presents the channel capacity in percent with respect to MU-mSISO. It can be observed that with MU-mMIMO $4 \times 4$ the highest channel capacity is gained with $220 \%$ and $280 \%$ more than in the MU-mSISO case at $P_{1}$ and $P_{2}$, respectively. The obtained capacity in the latter is higher due to its greater average SNR at $P_{2}$, which is $21 \mathrm{~dB}$ and only $16 \mathrm{~dB}$ at $P_{2}$ for MU-mMIMO $4 \times 4$. In the case of MU-mMIMO $2 \times 2170 \%$ at $P_{1}$ and $165 \%$ at $P_{2}$ is gained with respect to MU-mSISO. Even so the transmitted power for massive MIMO is $0 \mathrm{dBm}$ the obtained capacities are higher then in conventional MIMO configurations (see Fig. 5.3), where a transmit power of $10 \mathrm{dBm}$ is used. 


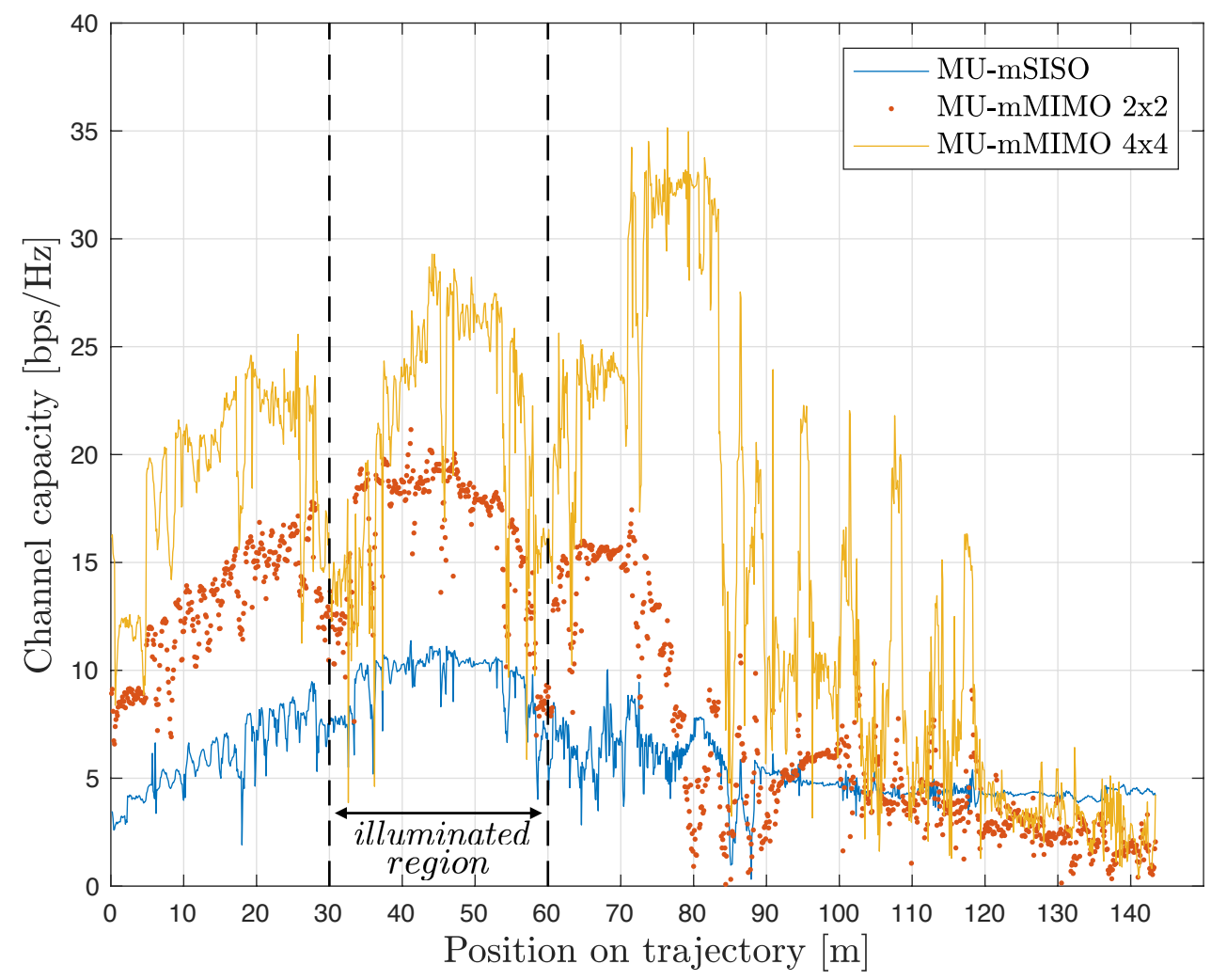

Fig. 5.9: Channel capacities for massive MIMO towards reference point $P_{1}$ at each position on the trajectory.

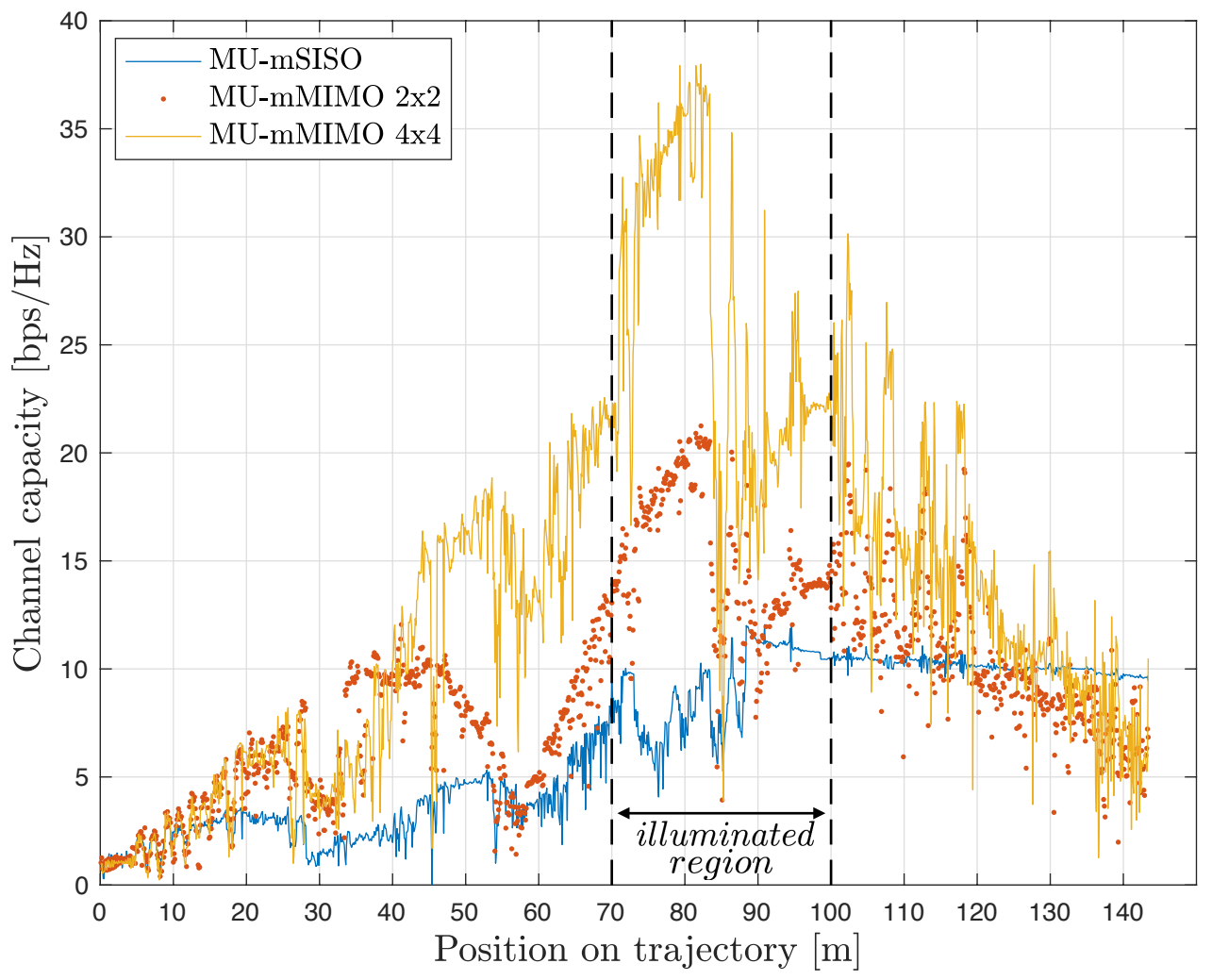

Fig. 5.10: Channel capacities for massive MIMO towards reference point $P_{2}$ at each position on the trajectory. 


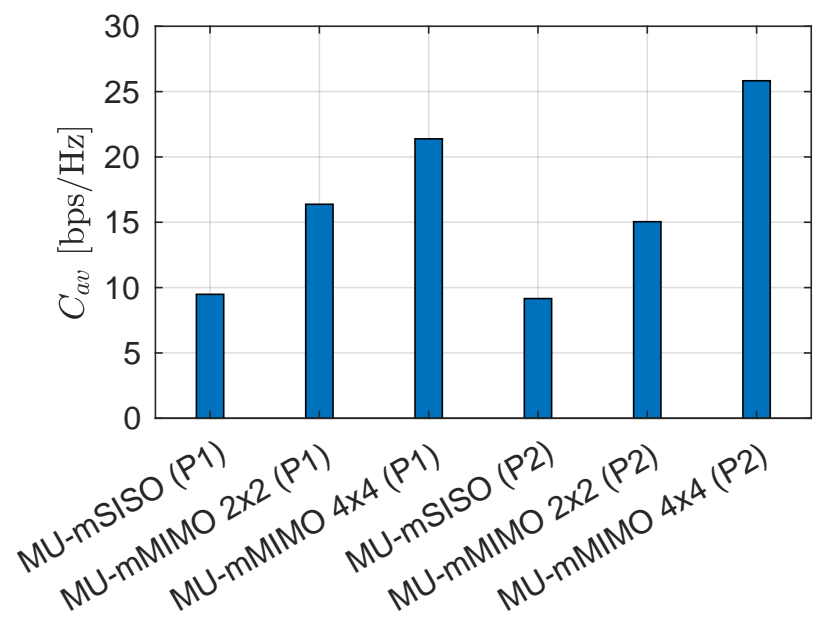

(a) Average channel capacity.

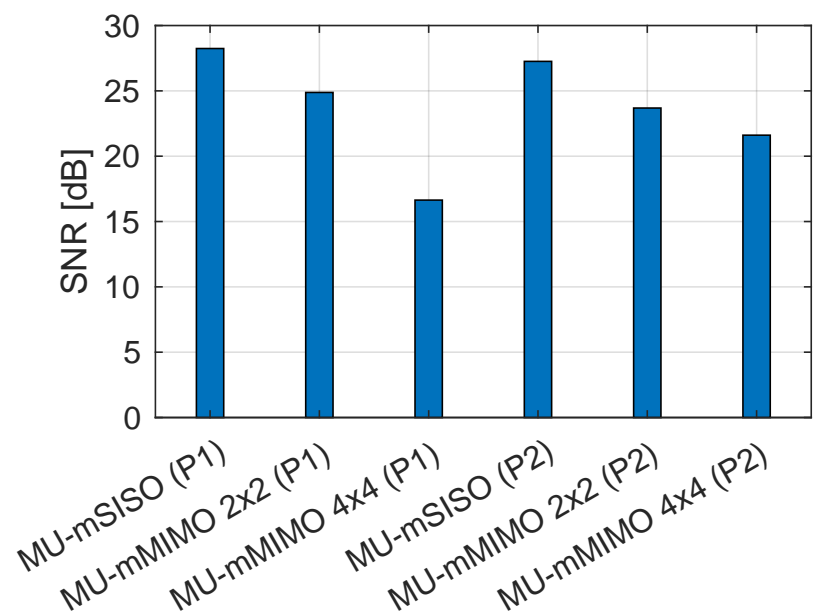

(b) Average SNR.

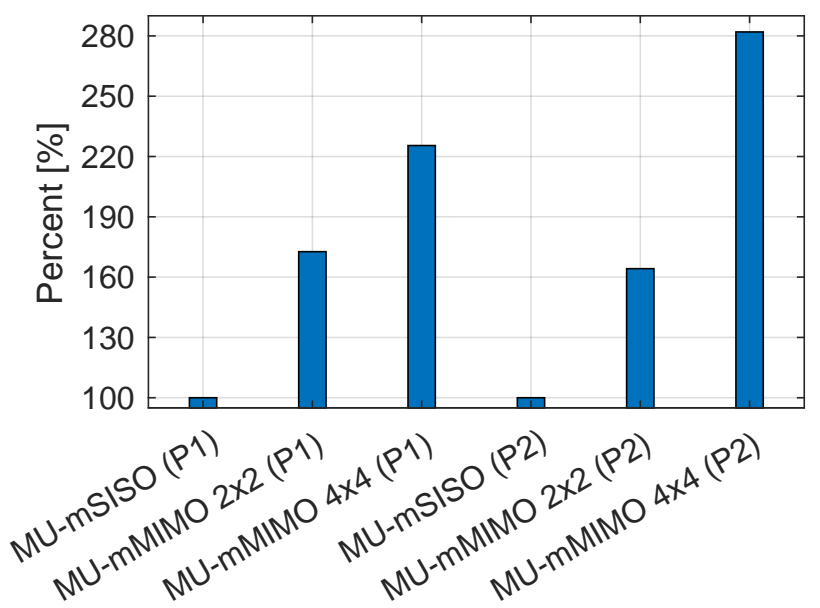

(c) Average capacity respect to MU-mSISO.

Fig. 5.11: Overview of the massive configuration average channel capacities and their corresponding average SNR when transmitting towards $P_{1}$ and $P_{2}$ with a constant overall transmit power of $0 \mathrm{dBm}$ at the BS. 


\subsection{Different Inter-element Spacing for Conven- tional MIMO}

In the following the different inter-element spacing is studied for conventional MIMO. The spacing between the monopole antennas on top of the car are selected to be between 0.1 up to 4 times the wavelength. The reason for investigating this behavior is that depending on the distribution of the angles of arrival a higher inter-element spacing is needed as explained in Section 2.2.3. Unlike previous studies no noise power level is selected but a fixed mean SNR of the trajectory. The mean SNR is for all obtained average channel capacities on the trajectory set to be $10 \mathrm{~dB}$. Fig. 5.12 shows the different obtained average channel capacities for distinct interelement spacing with respect to the corresponding SISO case in percent. Fig. 5.12a illustrates the MIMO 2x2 capacity in percent for the LOS in blue, the NLOS in red and the theoretical maximum in black. The same illustration is shown in Fig. 5.12b for MIMO $4 \mathrm{x} 4$.

Fig 5.12a shows MIMO 2x2 with the theoretical maximum channel capacity in percent of $149 \%$. The minimum for both LOS with $129 \%$ and NLOS with $135 \%$ is obtained with an inter-element spacing of 0.1 wavelength. The maximum with $147 \%$ for NLOS is achieved with a spacing of $3.4 \lambda$ and is almost reaching the theoretical maximum of $149 \%$. In the case of LOS the maximum of $139 \%$ although the channel capacity is almost not improving anymore for bigger inter-element distances as $1.9 \lambda$. The evolution for NLOS is expected as in the theory plot (see Fig. 2.4). The LOS can be subdivided into three plateaus. Fist LOS plateau goes from 0.4 to 0.7 , the second from 1.1 to 1.4 and then starting from 1.9 the channel capacity is getting flat.

In the following, MIMO 4x4 is analyzed with the theoretical maximum channel capacity in percent of $209 \%$. The lowest channel capacity is obtained for both LOS with $164 \%$ and NLOS with $175 \%$ with an inter-element spacing of 0.1 wavelength. The greatest capacity is achieved with an inter-element spacing of $2.8 \lambda$ and $3.9 \lambda$ and a obtained capacity of $188 \%$ and $198 \%$ for LOS and NLOS, respectively.

From the previous results, it is deduced that MIMO 2x2 is almost reaching the theoretical maximum for NLOS communication, whereas MIMO 4x4 has a bigger step until its maximum. It can be determined that the channel is not rich enough, even if the inter-element spacing is increased. Otherwise, it is also true that MIMO $4 \mathrm{x} 4$ provides a larger improvement with respect to SISO in terms of capacity although the channel rank is low.

Additionally, in MIMO 4x4 case, there is a more significant increment when the spacing is increased if the values are compared from the lowest distances to the largest. As stated in [15], "the specific behavior is also depending on the amount of elements in the car, which seems to indicate that higher is the number of elements, higher may be the necessary inter-element distance to obtain the optimal performance." 


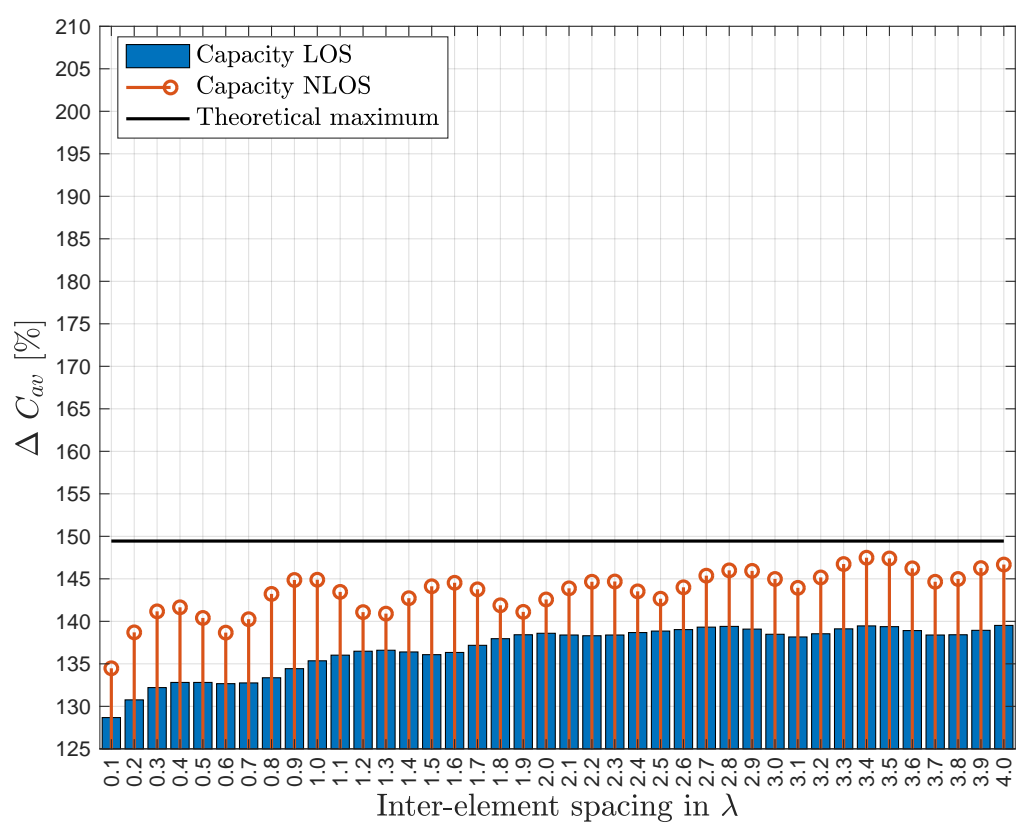

(a) MIMO 2x2 w.r.t. SISO.

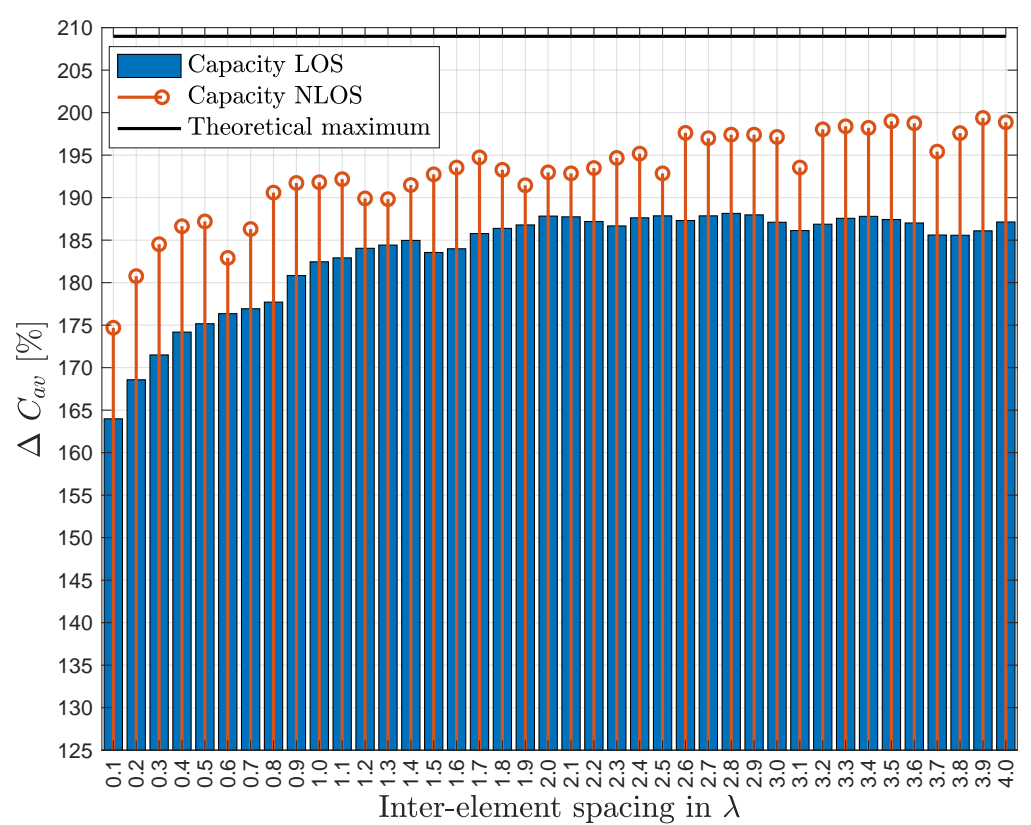

(b) MIMO 4x4 w.r.t. SISO.

Fig. 5.12: Average channel capacity for different inter-element spacing for MIMO $2 \times 2$ and $4 \times 4$ with respect to the SISO channel capacity in percent and a fixed mean SNR of $10 \mathrm{~dB}$. 


\section{Chapter 6}

\section{Conclusion}

This chapter concludes the presented research work of this thesis. Furthermore, potential directions for future work are proposed.

In this work, the impact on the eigenvalues and consequently on the channel capacity, by comparing different MIMO modalities, i.e., conventional MIMO and massive MIMO, was numerically analyzed. It is shown for the physical layer with a wave propagation based model that the usage of massive MIMO instead of conventional MIMO decreases the interference and improves the energy efficiency. Furthermore, the obtained results suggest that the channel capacity of a massive MIMO BS can be increased by $225 \%$ for a SNR of $16 \mathrm{~dB}$ by subdividing the massive antenna geometry into several channels in an urban environment in exchange for less focusing accuracy. Future work will focus on the investigation of the heterogeneity of eigenvalues for MU-mMIMO 2x2 and MU-mMIMO 4x4 and the relationship between this behavior and the number of elements at the massive BS.

In terms of antenna footprint, it has been shown the higher the order of MIMO system, the larger the inter-element distance needs to be to obtain an optimal performance. The increment of the distance improves capacity in both LOS and NLOS for V2I communications.

Further research is needed with experimental measurements. Therefore, a UWB prototype antenna was designed and measured, which works from 1.8 up to $35 \mathrm{GHz}$. This antenna allows to do measurements on top of a vehicle for certain configurations at different frequencies. 


\section{Bibliography}

[1] K. Abboud, H. A. Omar, and W. Zhuang, "Interworking of dsrc and cellular network technologies for v2x communications: A survey," IEEE Transactions on Vehicular Technology, vol. 65, no. 12, pp. 9457-9470, 2016.

[2] ACEA, "Frequency bands for v2x," ACEA Position Paper, no. 8, 2016.

[3] W. Viriyasitavat, M. Boban, H.-M. Tsai, and A. Vasilakos, "Vehicular communications: Survey and challenges of channel and propagation models," IEEE Vehicular Technology Magazine, vol. 10, no. 2, pp. 55-66, 2015.

[4] V. Jungnickel, K. Manolakis, W. Zirwas, B. Panzner, V. Braun, M. Lossow, M. Sternad, R. Apelfrojd, and T. Svensson, "The role of small cells, coordinated multipoint, and massive mimo in 5g," IEEE Communications Magazine, vol. 52, no. 5, pp. 44-51, 2014.

[5] A. Chelli, R. Hamdi, and M.-S. Alouini, "Channel modelling and performance analysis of v2i communication systems in blind bend scattering environments," Progress In Electromagnetics Research B, vol. 57, pp. 233-251, 2014.

[6] S. Wu et al., "Massive mimo channel modelling for $5 \mathrm{~g}$ wireless communication systems," PhD thesis, Heriot-Watt University, 2015.

[7] E. G. Larsson, O. Edfors, F. Tufvesson, and T. L. Marzetta, "Massive mimo for next generation wireless systems," IEEE Communications Magazine, vol. 52, no. 2, pp. 186-195, 2014.

[8] F. De Flaviis, L. Jofre, J. Romeu, and A. Grau, "Multiantenna systems for mimo communications," Synthesis Lectures on Antennas, vol. 3, no. 1, pp. 1$250,2008$.

[9] J.-C. Shen, J. Zhang, and K. B. Letaief, "Downlink user capacity of massive mimo under pilot contamination," IEEE Transactions on Wireless Communications, vol. 14, no. 6, pp. 3183-3193, 2015.

[10] J. Hoydis, S. Ten Brink, and M. Debbah, "Massive mimo: How many antennas do we need?" In Communication, Control, and Computing (Allerton), 2011 49th Annual Allerton Conference on, IEEE, 2011, pp. 545-550.

[11] C. Masouros, M. Sellathurai, and T. Ratnarajah, "Large-scale mimo transmitters in fixed physical spaces: The effect of transmit correlation and mutual coupling," IEEE Transactions on Communications, vol. 61, no. 7, pp. 27942804, 2013.

[12] R. Parolari, M. Gallo, A. P. Filisan, D. Zamberlan, V. Franchina, A. Michel, and P. Nepa, "A novel 3d antenna for lte mimo systems," in Electrical and Electronic Technologies for Automotive, 2017 International Conference of, IEEE, 2017, pp. 1-4. 
[13] Y. Hua, L. Huang, and Y. Lu, "A compact 3-port multiband antenna for v2x communication," in 2017 IEEE International Symposium on Antennas and Propagation USNC/URSI National Radio Science Meeting, Jul. 2017, pp. 639640. DOI: 10.1109/APUSNCURSINRSM. 2017.8072362.

[14] O. Y. Kwon, R. Song, Y. Z. Ma, and B. S. Kim, "Integrated mimo antennas for lte and v2v applications," in 2016 URSI Asia-Pacific Radio Science Conference (URSI AP-RASC), Aug. 2016, pp. 1057-1060. DOI: 10.1109/URSIAPRASC . 2016.7601146.

[15] A. Pfadler, C. Ballesteros, J. Romeu, and L. Jofre, "Multi-antenna configuration modeling for massive mimo v2i," in Antennas and Propagation (EUCAP), 2018 12th European Conference on, IEEE, 2018.

[16] B. Lindmark, "Capacity of a 2x2 mimo antenna system with mutual coupling losses," in Antennas and Propagation Society International Symposium, 2004. IEEE, IEEE, vol. 2, 2004, pp. 1720-1723.

[17] D. McNamara, C. Pistorius, and J. Malherbe, "The uniform geometrical theory of diffraction," Artech House, London, 1990.

[18] Z. Yun and M. F. Iskander, "Ray tracing for radio propagation modeling: Principles and applications," IEEE Access, vol. 3, pp. 1089-1100, 2015.

[19] R. G. Kouyoumjian and P. H. Pathak, "A uniform geometrical theory of diffraction for an edge in a perfectly conducting surface," Proceedings of the IEEE, vol. 62, no. 11, pp. 1448-1461, 1974.

[20] R. G. Gallager, Principles of digital communication. Cambridge University Press Cambridge, UK: 2008, vol. 1.

[21] C. E. Shannon, "A mathematical theory of communication," ACM SIGMOBILE Mobile Computing and Communications Review, vol. 5, no. 1, pp. 3-55, 2001.

[22] G. J. Foschini and M. J. Gans, "On limits of wireless communications in a fading environment when using multiple antennas," Wireless personal communications, vol. 6, no. 3, pp. 311-335, 1998.

[23] Á. C. Aznar, J. R. Robert, J. M. R. Casals, L. J. Roca, S. B. Boris, and M. F. Bataller, Antenas. Univ. Politèc. de Catalunya, 2004, vol. 3.

[24] D.-S. Shiu, G. J. Foschini, M. J. Gans, and J. M. Kahn, "Fading correlation and its effect on the capacity of multielement antenna systems," IEEE Transactions on communications, vol. 48, no. 3, pp. 502-513, 2000.

[25] W. C. Jakes and D. C. Cox, Eds., Microwave Mobile Communications. WileyIEEE Press, 1994, ISBN: 0780310691.

[26] Google, Institut cartografic de catalunya, https://www.google.com/maps / Q41 . 3955089, 2 .1700046 , 463a , 35y , 39 .35t/, Online; accessed 10 January 2018, 2017.

[27] WinProp, Winprop software for wave propagation and radio planning, http: / / www . altairhyperworks . com / product / FEKO / WinProp-Propagation Modeling/, Online; accessed 10 December 2017, 2017.

[28] Altair HyperWorks, Feko user manual, https://www.feko.info/download, version Version 2017. 
[29] M. Almarashli and S. Lindenmeier, "A new method for evaluation of lte mimo antennas in automotive application," in Antennas and Propagation (EUCAP), 2017 11th European Conference on, IEEE, 2017, pp. 2450-2453.

[30] A. De, T. K. Sarkar, and M. Salazar-Palma, "Characterization of the far-field environment of antennas located over a ground plane and implications for cellular communication systems," IEEE Antennas and propagation Magazine, vol. 52, no. 6, pp. 19-40, 2010.

[31] R. Ierusalimschy, Programming in lua. Roberto Ierusalimschy, 2006.

[32] J. J. van Tonder and U. Jakobus, "Fast multipole solution of metallic and dielectric scattering problems in feko," in Wireless Communications and Applied Computational Electromagnetics, 2005. IEEE/ACES International Conference on, IEEE, 2005, pp. 511-514. 


\section{Appendix A}

\section{Appendix}

\section{A.1 Conference Paper: URSI 2017}

The conference paper with the title: "Antenna Geometry Optimization for MIMO Connected Cars"; was accepted and presented at XXXII Spanish Symposium of URSI at Cartagena, Spain. 


\title{
Antenna Geometry Optimization for MIMO Connected Cars
}

\author{
C. Ballesteros, A. Pfadler, L. Jofre, J. Romeu \\ christian.ballesteros@tsc.upc.edu, andreas.pfadler@tsc.upc.edu, jofre@tsc.upc.edu, romeu@tsc.upc.edu \\ Signal Theory and Communications Department. Universitat Politecnica de Catalunya. UPC Campus Nord, 08034 Barcelona.
}

\begin{abstract}
Connected cars are becoming one of the fastest developing scientific and technological territories. The need for large capacities and small delay times are forcing the proper electromagnetic characterization of the connected car environment. In order to have realistic approaches, the specific antenna, the car geometry and the urban environment need to be adequately modeled. In this study, a numerical tool is used to obtain a robust basis for the optimization of the three elements. In a first step, the electromagnetic parameters of different positions of a patch antenna on the roof of a car and also on a fixed Base Station (BS) will be obtained. Them, those parameters will be jointly combined to compare three different constellations. The primary configuration is a Single-Input Single-Output (SISO) system using one patch antenna on both the car and the BS. The secondary SISO system is comprised of an array of 16 patches at the BS. The third constellation is utilizing the same patch array geometry for the BS but operating as a Multiple-Input and Multiple-Output (MIMO) system involving four patch antenna placed on the roof of the vehicle.
\end{abstract}

\section{INTRODUCTION}

The interest of the global society in telecommunications has experienced a very important growth the last years and even more from the beginning of the 2010 decade. Everybody wants to be connected to others and also to everything, even to cars. This interest is not just for simple communication purposes, but also for safety and it is a key factor in the new era of the automotive industry. In this scenario, there are many unknowns still pending to be solved and further analysis are required before applying any solution.

Regulation entities have defined some standards, with the corresponding frequency band allocation in the overcrowded spectrum [1] [2], as a starting point in order to develop new technologies. On one hand, the Electronic Communications Committee (ECC) and the European Union defined a $30 \mathrm{MHz}$ band at $5.9 \mathrm{GHz}$, which was finally integrated in the IEEE 802.11p standard for Vehicle-to-Everything (V2X) applications. Otherwise, the automotive industry is trying to follow another approach, focusing on lower carrier bands with better propagation properties for mobile communications: between $3.4-3.8 \mathrm{GHz}$. This band has been chosen for the design of the model and the simulations that will be presented in this document due to the lesser amount of research and specifications on it for connected cars.

Another important topic in modern communications is the introduction of MIMO structures [3] [4], which allow an important increase in terms of capacity. The demanding requirements in $\mathrm{V} 2 \mathrm{X}$ applications regarding to small latency and high rates have led to the use of multiple antennas both in vehicles and base stations. The study of this environment is necessary to understand the behavior of already known techniques in new situations with many possible variations and, moreover, to predict the feasibility of upcoming implementations. Therefore, this documents pretends to present an approach to a particular model of communication between cars and BS using a commercial tool for electromagnetic simulation.

The paper is organized as follows. Section II describes the system model that is used to analyze the efficiency of the SISO and MIMO systems for the V2X communication. Section III provides the details of the FEKO simulation software, from Altair company, used for the analysis and also other required tools. Section IV presents the used methodology for the analysis. In section $\mathrm{V}$ the comparison results of the two SISO constellations and the MIMO systems are demonstrated and discussed. Finally, conclusions are presented in Section VI.

\section{System MODEL}

It is widely known that one of the main issues in mobile communications is related to its modeling. Sometimes, it is difficult to know the theoretical behavior and simulation is the only tool to obtain the required parameters. In this case, a model must be defined taking into account all the possible variants.

For the case of study in this work, a car with roof window, provided by [5], is placed in the middle of a street, jointly with a BS. Due to computational limitations and for simplification of the model, only the metallic body and the windows are considered. The body consists of steel sheets of $2 \mathrm{~mm}$ of thickness [6], while windows are made of layered glass panels of less than $6 \mathrm{~mm}$ [7]. A wall is located at one side of the road, at the same distance from both the user and the BS, to imitate building on the street made of regular bricks, whose dielectric properties have been provided by Altair's material database for WinProp software (another tool for EM propagation and network design). Finally, the street pavement is made with an asphalt ground plane [8].

The basic element used for the communication is a patch antenna with an squared base of $\lambda / 2$ per side. It is loosely adapted to work at $3.6 \mathrm{GHz}$ using a pin feeding point connected to a $50 \Omega$ voltage source. The same structure is used for the user and the BS. A planar structure for the BS, parallel to the $\mathrm{XZ}$ plane, is chosen and the antennas are pretended to be evaluated in basically three configurations: one element in the central position, a $4 \times 4$ array and 4 independent arrays of 4 elements each but using the same $4 \times 4$ arrangement than before. On the other hand, five patch antennas are placed on the roof of the car. 2 depicts their exact position. In this case, 


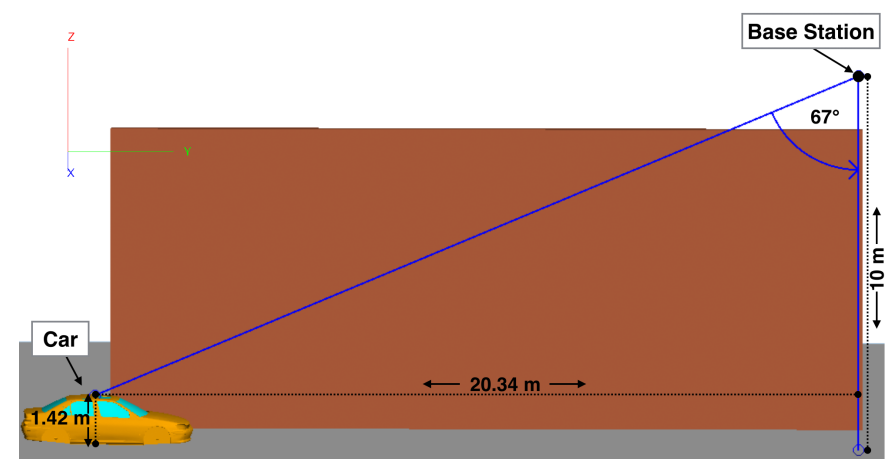

Fig. 1. Scenario representation

the four patches are faced up, pointing with their main beam in the positive direction of the $\mathrm{z}$ axis. The scenario is presented in Figure 1.

More assumptions have been introduced to the model to adjust the calculations, such that the results obtained can be comparable to a real situation:

- The distance between the center of the car basis and the projection of the BS on the XY plane is $20 \mathrm{~m}$. The height of the car roof, where the antennas will be located, is $1.42 \mathrm{~m}$ and the center of the $\mathrm{BS}$ is $10 \mathrm{~m}$ over the ground plane.

- Constant power is delivered to the transmitter, so the summation of all sources is not exceeding a given value. Given the distances considered and, in order to get a reasonable $\mathrm{SNR}$, a total power of $1 \mathrm{~mW}(0 \mathrm{dBm})$ and a noise floor of $-80 \mathrm{dBm}$ are considered.

- The basic antenna for both the car and the BS consists into a patch antenna over a Perfect Electric Conductor (PEC) based on [5] guidelines, with a dielectric substrate whose relative permittivity is $\epsilon_{r}=2.55$.

- Free space is considered for $z \geq 0$ and asphalt, with dielectric properties taken from [8], for $z<0$.

\section{Simulation TOOL}

In order to investigate different BS geometries and to find the most appropriate antenna placement on the car, it is used the FEKO software suite that provides geometric elements, basic antennas and includes a Multilevel Fast Multipole Method (MLFMM) that is an alternative conceptualization of the Method of Moments (MoM). This method offers great possibilities and flexibility in terms of simulation time. The tool provides a variety of other solvers, which have not been considered in this work. For more details about the simulation tool, the reader is encouraged to refer to [5].

The main reason to use this software consists in simulating the far field radiation patterns for both BS and car antennas, considering multiple geometries. As the final target is to calculate the channel matrix and, in consequence, its associated capacity in order to be optimized, a second tool is required to perform the propagation analysis. Altair company also includes another software suite for EM analysis: WinProp. It contains all the tools necessary to obtain an accurate analysis of the radio channel and even deploy a network with different cellular technologies. Then, importing the radiation patterns

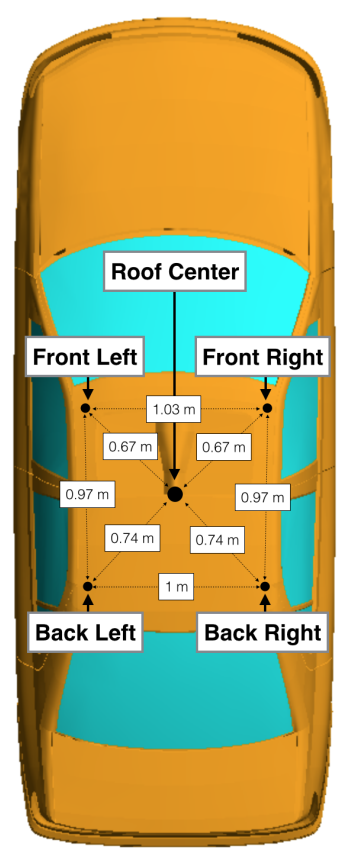

Fig. 2. Placement of the four patch antennas for the MIMO case and the location (Roof Center) for the two SISO cases.

previously calculated with FEKO, it is possible to obtain the channel matrix for a particular scenario.

Otherwise, before submitting this article, WinProp features have not been deeply investigated and will be relegated until future analysis. In consequence, MATLAB software has been chosen for the channel matrix calculation once the car and the BS models have been simulated with FEKO, using the ray-tracing approach as WinProp models do.

\section{Methodology}

If an improvement is wanted to be measured with different configurations and systems, it is required to first define a figure of merit and a procedure to calculate it using the available tools.

In this particular scenario, capacity will be the magnitude to evaluate the performance of each design. For that, the channel matrix is required and two paths can be defined in order to obtain it: an analytic expression with a simplified multipath model, considering only a direct ray and one or two main reflected paths, or a matrix based on the simulated values at each element which provide magnitude and phase of the field received in the antenna. In particular, the receiver is loaded with $50 \Omega$ at each port (element output). The software provides any measure at the loads and, for this case, voltage is chosen because it is the same magnitude that is controlled by the transmitter to insert the weights.

The capacity for a MIMO system may be obtained as:

$$
C=\log _{2}\left(\operatorname{det}\left[I_{N r}+\frac{P_{T} H H^{*}}{P_{N}}\right]\right)
$$

where $H$ is the $N r \times N t$ channel matrix, $P_{T}$ and $P_{N}$ are transmitted and noise power, respectively, and ( $)^{*}$ denotes the transpose conjugate. Otherwise, the expression can be defined as a function of the eigenvalues resulting from the product between $H$ and its transpose conjugate. A new parameter 
is defined as $N=\min \left(N_{T}, N_{R}\right)$, the maximum number of simultaneous information flows that can be sent in a MIMO system.

$$
C=\sum_{i=1}^{N} \log _{2}\left(1+\frac{P_{T} \lambda_{i}}{P_{N} N}\right)
$$

The role of the channel is essential to understand the limitations of a communications system and its variability must be analyzed to reach trustworthy conclusions. Due to the need for starting the investigation, only static simulations are feasible but multiple positions of the car respect to the BS can be analyzed with several iterations. The effect of moving objects and its impact in the wave propagation, is omitted but this is an adequate solution to obtain some initial knowledge of the system.

The channel matrix includes several parameters to consider when it is calculated. First, the radiation pattern of both the receiving and transmitting antennas. Obviously, frequency affects the path loss, this is the main reason to work at the lowest value possible. Choosing the $3.6 \mathrm{GHz}$ band would suppose an improvement respect to the band around $5.9 \mathrm{GHz}$.

Reflections on the ground or the walls are really important, as MIMO benefits of it to gain in performance. Otherwise, this scenario is not good when only one data path (SISO) is wanted. In the case of using patch antennas pointing in the same direction as the $\mathrm{z}$ axis, we are avoiding any reception from waves reflected in the ground if no other element is considered above the car. Then, those reflections will not be taken into account in theoretical calculations. In general, the channel matrix can be defined as the one obtained using the direct path between all elements and the summation of the contribution of each reflected path considered, as here stated:

$$
H=H_{\text {direct }}+\sum_{k=1}^{K} \rho_{k} H_{k}
$$

where $\rho_{k}$ represents the reflectivity of the material associated to the multipath reflection ' $k$ ' respect to the free space (air). For simplicity of the model, only the direct path and one reflection on the wall will be considered in later calculations.

\section{CASE OF Study}

The transmitted power of $1 \mathrm{~mW}$ is used for all cases. Fig. 2 shows the different placements of the patch antennas on the top of the car, which are used in two configurations: only one antenna, next to the roof window, when a single output is wanted or four antennas on the corners to be used as multiple outputs. Regarding to the BS, always 16 elements are used, except in the first case, where only one is placed to obtain a reference value of the capacity without implementing any array or MIMO system.

\section{A. SISO: patch-to-patch communication}

For this geometry, the BS consists of one patch antenna and it is communicating with the patch "Roof Center". The maximums of radiation of both antennas are orthogonal between them, but the different heights of each element allow to receive a minimum amount of power. Figure 3 shows the radiation pattern of a single patch antenna in linear scale.

For this first case, a simple point to point system is calculated and the radiation of transmitter and receiver are
Total Gain

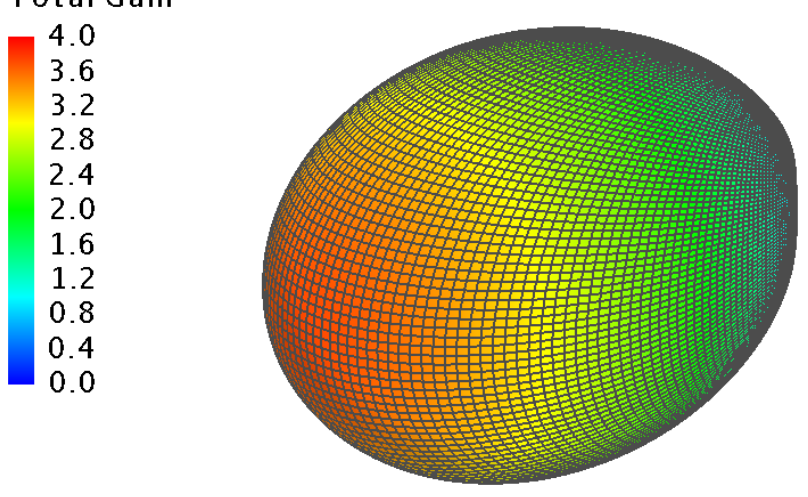

Fig. 3. Patch antenna radiation pattern

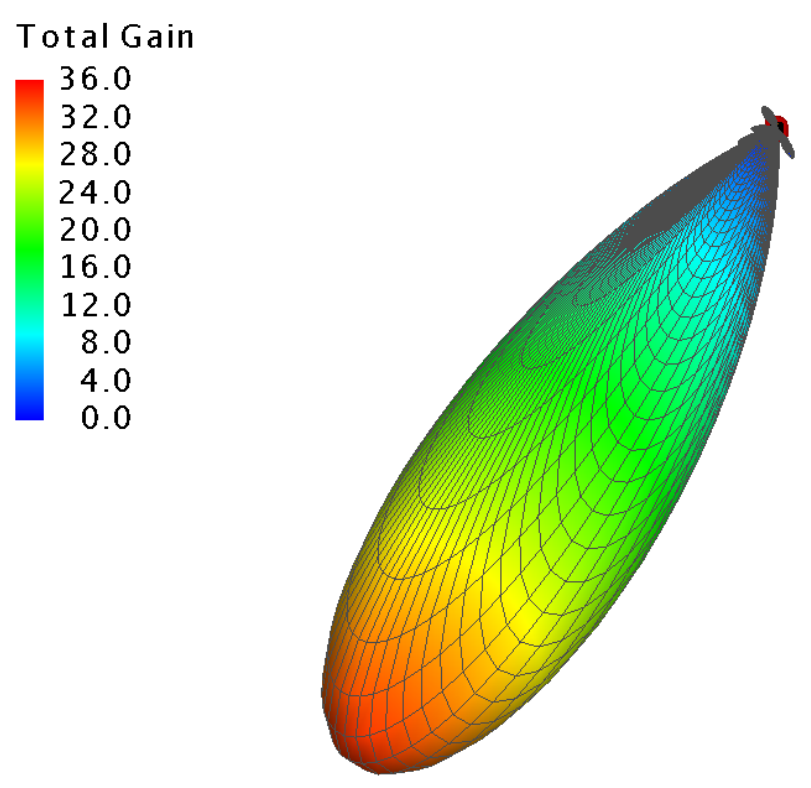

Fig. 4. 16 elements beamforming radiation pattern

is only depending on the patch element. Due to the specific position of both antennas, the signal reception is no optimal and the resulting capacity is $C_{S I S O_{-} \text {single }}=0.865 \mathrm{bps} / \mathrm{Hz}$.

\section{B. SISO: 16 patch-array array beamforming}

The second SISO case, introduces the concept of beamforming with a $4 \times 4$ array at the BS with the same source power as section A, but scaled depending on the weights of each element. The single patch antenna on the top of the car is also the same as before, placed in the "Roof Center" point.

Now, as can be seen in Figure 4, the array is concentrating its radiation capabilities in one specific direction, which is determined with a DOA calculation algorithm based on a pilot signal received from the same antenna mounted on the car. In this case, the system is still using one single transmitter but on a much smarter way, using more elements too. In this case, the capacity achieved is $C_{S I S O \_m u l t i}=3.346 \mathrm{bps} / \mathrm{Hz}$. 


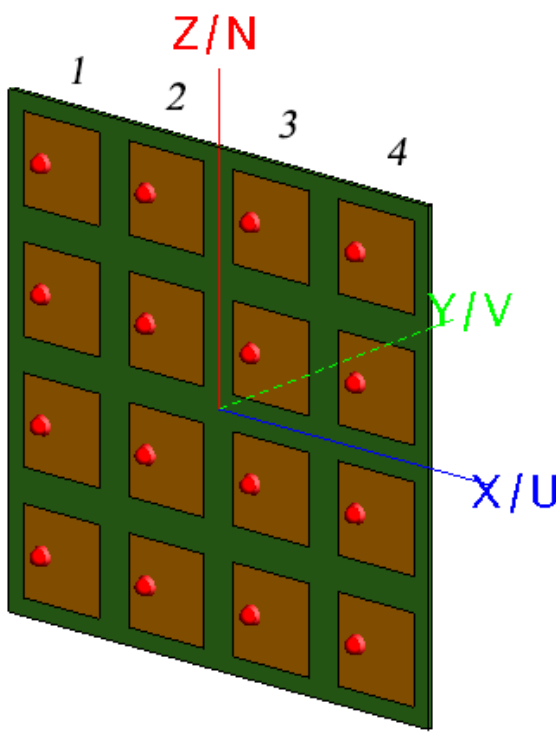

Fig. 5. Column-wise division of the BS

\section{MIMO: 4 into car and 16 into BS}

Finally, in the third case studied for this document, the BS is divided column-wise into four groups of four antennas, which are focused towards one different patch on the top of the car. Then, both MIMO and beamforming techniques are applied to, hopefully, improve the system performance. The beam of the first column is pointing to the patch location "Back Left", the second column of the BS towards the "Front Left" patch. The third column is focused to the "Front Right" and the fourth towards the "Back Right".

The main problems of using this kind of configurations with antennas that are quite close are the correlation of the fields, which affects the channel matrix, and the interference generated between antennas. Then, MIMO systems should ensure that all the elements are enough separated in order to obtain better results and this is the reason to locate the antennas on the four roof corners. In this case, the final capacity is $C_{M I M O}=2.928 \mathrm{bps} / \mathrm{Hz}$.

For this particular case, the usage of multiple parallel transmissions is not improving the capacity. This effect is due to the fact that we are using the same number of elements in the single array and in the MIMO structure, just to get a fair compairison. Otherwise, this does not seem a good solution compared to beamforming if the number of elements is kept as constant because, even increasing the simultaneous data flows being sent, the system is loosing gain related to the array pointing accuracy.

Finally, it is mandatory to mention that the three cases could be better compared in terms of capacity if power is not a restriction but a parameter. In figure 6 , capacity values are shown as a function of the total source power, which was one of the inputs for the simulation. This parameter, represented in logarithmic scale on the horizontal axis, will define the final Signal-to-Noise Ratio (SNR) at each receiver (if more than one is present) and therefore the final capacity. As can be seen, for larger values of power, MIMO configuration improves faster than Massive SISO and should be considered as a better

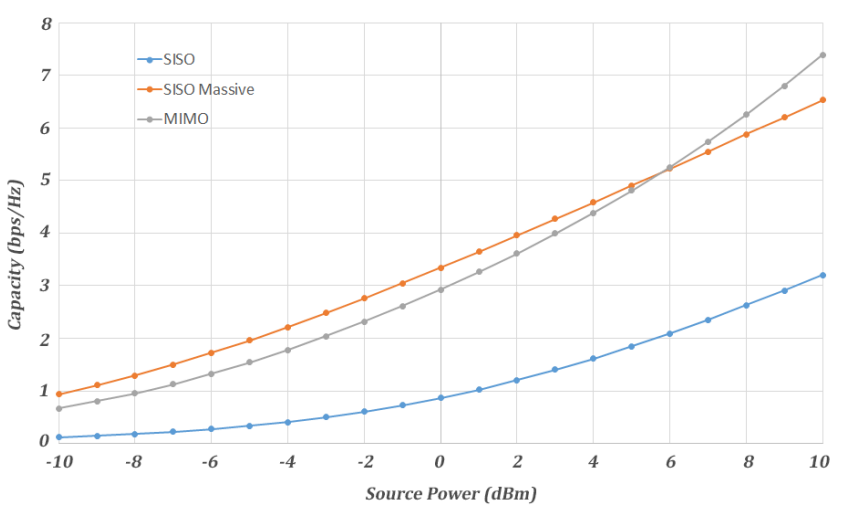

Fig. 6. Capacity depending on source power

option. Then, this graph is verifying the strict relation between the available resources and the scenario with the preferable method to choose. Thus, further investigations are required to define a structure for the purpose of connected cars and it means a better knowledge of the real environment.

\section{CONCLUSIONS}

A very first comprehensive model of a realistic car to BS (V2I) system has been modeled in order to be able to optimize both the Base Station (Massive) and the antenna car multielement MIMO geometries. The very first results support the intuitive results on the interest on increasings the number of element into both car and the BS multi-antenna geometries. On the final presentation, more specific parameters for the antenna location based into the system capacity obtained from the realistic channel matrices will be presented.

\section{ACKNOWLEDGEMENTS}

This work was supported by the Spanish "Comision Interministerial de Ciencia y Tecnologia" (CICYT) under projects TEC2013-47360-C3-1-P and TEC2016-78028-C3-1$\mathrm{P}$ and Altair FEKO HyperWorks.

\section{REFERENCES}

[1] European Automobile Manufacturers' Association (ACEA), "Frequency Bands for V2X," ACEA Position Paper, Dec. 2016.

[2] K. Abboud, H. A. Omar and W. Zhuang, "Interworking of DSRC and Cellular Network Technologies for V2X Communications: A Survey," IEEE Transactions on Vehicular Technology, Vol. 65, Num. 12, Dec. 2016.

[3] J. Karedal et al., "Measurement-based modeling of vehicle-to-vehicle MIMO channels," IEEE International Conference on Communications, pp. 3470-3475, Jan. 2009.

[4] D. Quack et al., "Simulation-based Evaluation of MIMO Antenna Systems in Car-to-Car Communication," 8th European Conference on Antennas and Propagation (EuCAP 2014), Apr. 2014.

[5] Altair HyperWorks Version 2017, FEKO User Manual, Jan. 2017.

[6] R. Rana, S. B. Singh, Automotive Steels: Design, Metallurgy, Processing and Applications, Woodhead Publishing, 2016.

[7] G. W. McLellan and E. B. Shand, Glass Engineering Handbook, 3rd ed., McGraw-Hill, 1984.

[8] E. J. Jaselskis, J. Grigas and A. Brilingas, "Dielectric Properties of Asphalt Pavement," in Journal of Materials in Civil Engineering, vol 15, Num. 5, September/October 2003, pp. 427-434. 


\section{A.2 Conference Paper: EuCAP 2018}

The conference paper with the title: "Multi-antenna Configuration Modeling for Massive MIMO V2I"; is accepted and will be presented at the 12th European Conference on Antennas and Propagation at London, United Kingdom. 


\title{
Multi-antenna Configuration Modeling for Massive MIMO V2I
}

\author{
A. Pfadler, C. Ballesteros, J. Romeu, L. Jofre \\ Signal Theory and Communications Department, Universitat Politecnica de Catalunya, Barcelona, Spain, e-mail: \\ $\{$ andreas.pfadler, christian.ballesteros, jofre, romeu $\} @$ tsc.upc.edu
}

\begin{abstract}
There is a growing research interest in massive Multiple Input Multiple Output (MIMO) antenna systems because of their higher channel capacity and energy efficiency. New applications in digital mobility and connected car is certainly one of the areas of future development of these systems. In order to study the impact of the different multi-antenna geometries and MIMO modalities in both the vehicle and the fixed base station, a quality modeling of a realistic scenario is needed. In this paper, study of initial antenna configurations for both - mobile and fixed - platforms is performed and initial channel parameters and system capability are obtained. Then, several systems and constellations are compared by means of the channel eigenvalues and capacity. The analysis is based on the results of a simulated model of an urban scenario, emulating as a case of study a portion of the city of Barcelona in a realistic environment, comprising of a massive MIMO base station and a car at various positions.
\end{abstract}

Index Terms-V2I Communications, Massive MIMO, Beamforming, Channel Modeling, Software Simulation, Urban.

\section{INTRODUCTION}

Telecommunications industry has put its focus on Vehicleto-Everything (V2X) applications in recent years due to the increase in interest of users and manufacturers towards a more comfortable and secure driving. Furthermore, the academic researchers as well as the industry are nowadays driving their attention towards the new technological innovations in massive MIMO systems. Its benefits have largely been demonstrated and the inclusion of multi-antenna constellations with beamforming capabilities in mobile communications has opened a wide area to investigate. In this document, the analysis will be centred on the physical layer for several antenna configurations mounted on a car in an urban environment. Due to the complexity of the system, numerical methods are required and this implies the use of simulation software tools. Altair's FEKO, for the radiation patterns of both the car and Base Station (BS) antennas, and WinProp suite, for the propagation analysis in the urban environment, are the two main software tools used to obtain the results.

Regulation entities have defined some standards, with the corresponding frequency band allocation in the overcrowded spectrum [1] [2], as a starting point in order to develop new technologies. On one hand, the Electronic Communications Committee (ECC) and the European Union defined a $30 \mathrm{MHz}$ band at $5.9 \mathrm{GHz}$, which was finally integrated in the IEEE 802.11 p standard for V2X applications. On the other hand, the automotive industry is trying to follow another approach, focusing on lower carrier bands with better propagation properties for mobile communications: between $3.4-3.8 \mathrm{GHz}$. In this work, the latter band is chosen for the design of the model since it has not been researched previously and consequently there is a lack of specifications on it for connected cars.

This work makes the following contributions:

1) Evaluation of different MIMO modalities with a Massive BS for Vehicle-to-Infrastructure (V2I) communication in an urban environment.

2) The impact on the channel capacity by changing the inter element spacing of the patch antenna array on the rooftop of a vehicle.

The paper is organized as follows. Section II describes the complete system, including all elements composing the entire scenario (i.e. urban environment, antenna elements, user car and BS), for which the channel will be identified. Then, Section III presents the theoretical background and analytic tools which will be used to evaluate next simulation results. Section IV provides the details of the requested outputs in the simulation software and also a comparison between various configurations is discussed based on previous definitions. Finally, conclusions are presented in Section V.

\section{System ModeL}

The system model is presented in this section and consists of the urban environment, BS configuration and vehicle with multi-antenna setup and software tools used for the simulation.

\section{A. Urban Environment}

In order to obtain a more realistic approach for the analysis of a car in an urban area, a portion of a real city has been chosen to simulate the V2I environment. In particular, Fig. 1 depicts an intersection in the city of Barcelona, corresponding to the Eixample (a district characterized by its rectangular shapes and almost 90 degrees corners). The environment is comprised of a ground plane made of asphalt, five buildings, three of them with courtyards, traffic lights and trees. The height of all buildings is 18 meter and the BS will be placed on the top of one building at height of 21 meters, 3 meters above the rooftop. $B S_{x}$ is one of the three BS configurations (see also Fig. 4) and there are three reference points, $P_{1}$ to $P_{3}$, for the location of the car. These points represent the still position of the vehicle. Therefore, the direction of travel is from $P_{1}$ to $P_{3}$. The distance between $\mathrm{BS}$ and the reference 


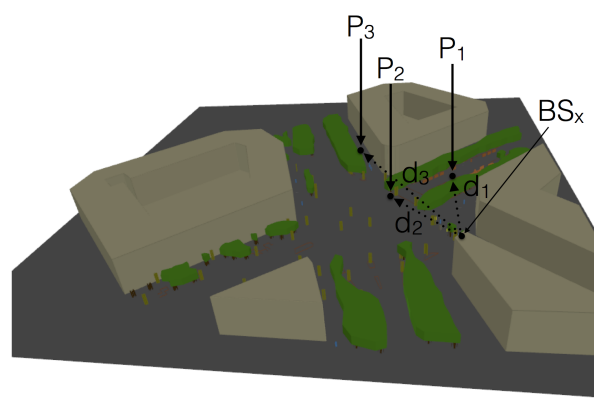

Fig. 1. The scenario represents an urban intersection of Barcelona including the position of the BS and the distance $d_{1}$ to $d_{3}$ towards three positions of the car in the street $P_{1}$ to $P_{3}$.

points is represented by $d_{1}$ to $d_{3}(107.8,86.9$ and 118.2 meter, respectively).

All buildings are modeled as 3D objects with material properties that influence the reflection of the rays on their surface. On the other hand, trees are made of wooden trunk and vegetation top, which lets the rays to pass at the cost of an additional attenuation.

\section{B. Vehicle Multi-antenna Setup}

Up to nine different configurations have been considered for the antennas on the car roof. The left hand side of Fig. 2 depicts the vehicle with a fixed reference point. The car body is assumed to be made of metal and the windows of laminated glass. The same figure shows the position of the patch antennas for each setup with respect to the common reference. In the case of only one antenna, it is located at this point. Then, two more cases will be analyzed: two and four patch antennas. For each one, the inter-element spacing is successively doubled from $\lambda / 2$ until $4 \lambda$ ( $\lambda$ is the operation wavelength), maintaining the same distance to the reference. For the case of two antennas, they are placed in the front side and not in the middle for two reasons: the same positions are kept respect to the four-element case and it is possible to see more clearly the difference between the illumination of the antennas when the car moving towards or away from the BS.

\section{Base Station}

Regarding the transmitter, a BS composed of 64 patch antennas is considered. Fig. 4 shows three different configurations, depending on the number of input channels, but keeping the total number elements at the BS constant. $B S_{1}$ is using all antennas to create a beam pointing to the reference points. It is used for the Single Input Single Output (SISO) and Single Input Multiple Output (SIMO) case. Fig. 3 illustrates the power map for a single input case at a height of the car rooftop, when the $\mathrm{BS}$ is transmitting to $P_{1}$. Then, $B S_{2}$ is subdivided in two arrays of $4 \times 8$, reducing the focusing capabilities of each channel to only 32 elements. For the case of MIMO 2x2 and Multiple Input Single Output (SISO) 2x1. Finally, the third configuration, $B S_{3}$ is composed of four $4 \times 4$ antenna

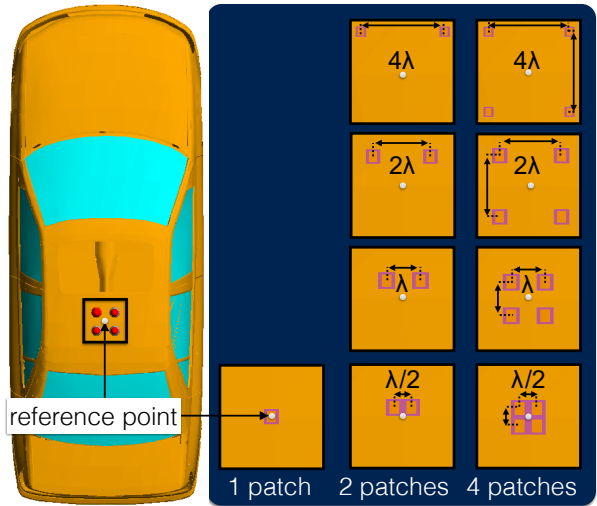

Fig. 2. Overview of the different patch antenna setups with one, two and four patch antennas on the rooftop of the car.

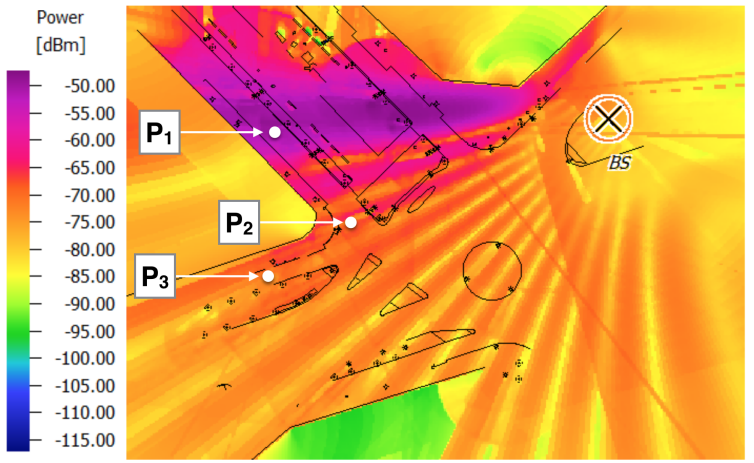

Fig. 3. Power map at a height of the car rooftop (1.42 meter), when the BS transmits to $P_{1}$ acting as single input.

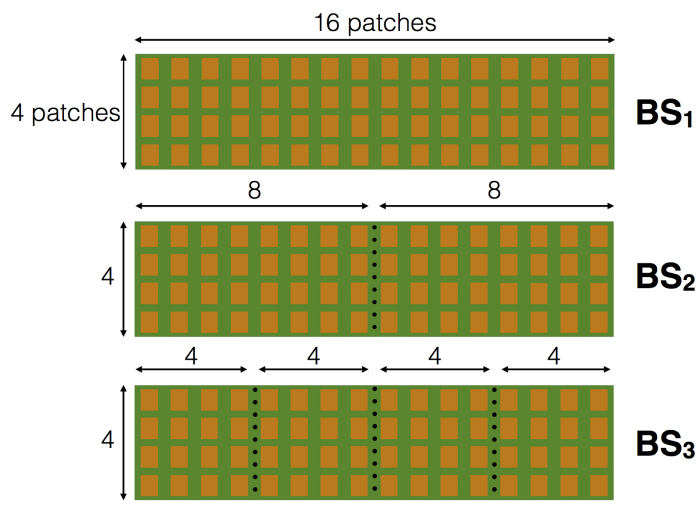

Fig. 4. Base Station with 64 patch antennas and 3 configurations: massive $4 \times 16$, two times $4 \times 8$ and four times $4 \times 4$.

arrays. Four beams are created, with a wider width, and they are used in MIMO $4 \times 4$ and SISO $4 \times 1$. In order to steer the beam towards a certain position, a proper magnitude (assumed uniform for all cases) and phase is set for each element. The weights are calculated in advance for each of the three positions of the car. 


\section{Simulation Tools}

In order to evaluate different geometries and perform a propagation analysis for the actual scenario, the following tools are in use. First, the antenna weights for the BS are calculated with a Matlab script. In second term, the antenna geometry is created and simulated, with previously obtained weights, to obtain its radiation pattern using Altair's FEKO [4]. This is done for each BS configuration and car setup, taking into account the impact of the electromagnetic properties of the car materials. Finally, the generated pattern is imported in WinProp [3]. There, a 3D ray tracing method is used to calculate the channel matrix, with empirical values of the losses in transmissions, reflections and diffractions for all materials composing the objects.

\section{Methodology}

The capacity for a MIMO system may be obtained as [5]:

$$
C=\log _{2}\left(\operatorname{det}\left[I_{N r}+\frac{P_{T} H H^{*}}{P_{N}}\right]\right)
$$

where $H$ is the $N r \times N t$ channel matrix, $P_{T}$ and $P_{N}$ are transmitted and noise power, respectively, and ( $)^{*}$ denotes the transpose conjugate.

Additionally, the expression can be defined as a function of the eigenvalues $\sigma_{i}$ with $i$-th element of a matrix resulting from the product between $H$ and its transpose conjugate. Also a new parameter is defined as $N=\min \left(N_{T}, N_{R}\right)$, being the number of non-null eigenvalues (matrix rank) or, in terms of communications, the maximum number of simultaneous information flows that can be sent in a MIMO system.

$$
C=\sum_{i=1}^{N} \log _{2}\left(1+\frac{P_{T} \sigma_{i}}{P_{N} N}\right)
$$

This relationship is essential to analyze the simulation results.

\section{Simulation Results AND ANALYsis}

In this section the simulation settings and the procedure of analyzing the results is discussed and finally the simulations results are presented.

\section{A. Initial assumptions}

There are conditions that have to be defined at the beginning, due to their impact on the final results. The most evident case is the Signal-to-Noise Ratio (SNR). This parameter has a strong influence in the capacity calculation and power levels have been adjusted to achieve values close to real situation for automotive MIMO applications [6], around 10-20 dB. Also the number of interactions with the objects in the scenario has been fixed to prevent from very large computation times.

- BS total transmission power: $P_{T}=10 \mathrm{dBm}$

- Noise level: $P_{N}=-75 \mathrm{dBm}$

- Total area of scenario: $275 \times 250 \mathrm{~m}$

- Car antennas height: $1.42 \mathrm{~m}$

- BS center height: $21 \mathrm{~m}$

- Buildings height: $18 \mathrm{~m}$

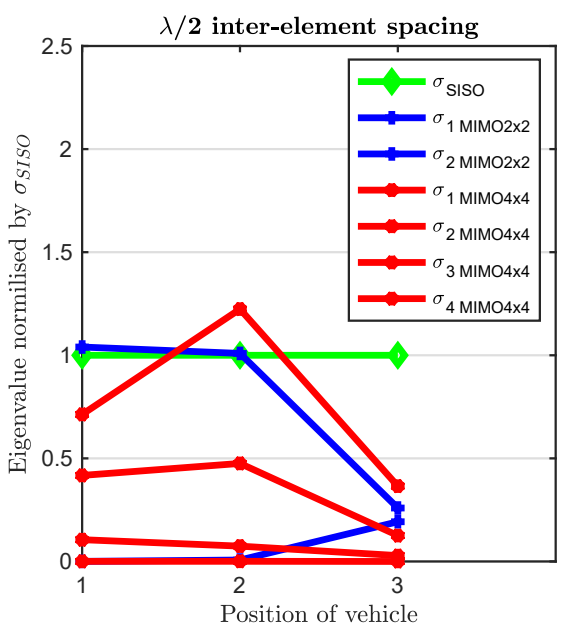

(a)

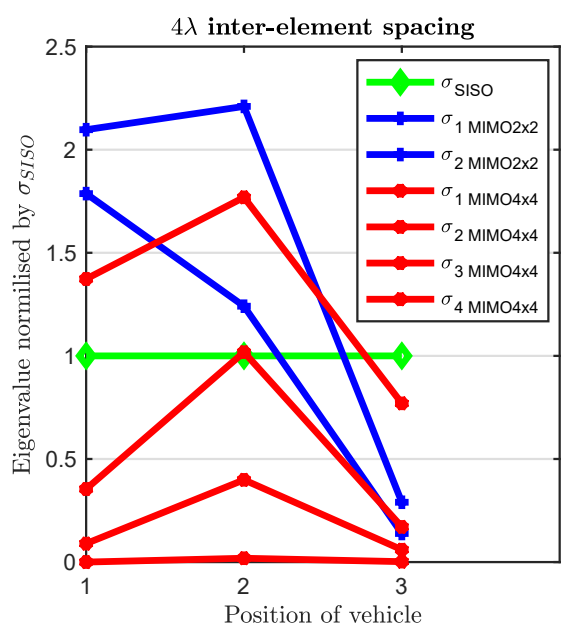

(b)

Fig. 5. Normalized eigenvalues (to $\sigma_{S I S O}$ ) for each car position with interelement spacing of $\lambda / 2$ and $4 \lambda$ for all setups.

\section{B. Outputs and Figures of Merit}

Based on the obtained channel matrix, the comparison of the different setups and MIMO technologies is done by studying two figures of merit: channel eigenvalues and capacity, already defined in section III. The channel capacity can be directly compared for each case, while eigenvalues are normalized for each position of the car by the corresponding eigenvalue $\sigma_{S I S O}$ of the massive SISO case.

In general, the target of a communications system is to maximize the amount of delivered information from one end to the other. Therefore, larger capacity will imply higher performance, but other aspects must be taken into account. Almost equal eigenvalues are synonymous with a similar use of all inputs and outputs, then, the increase in the number of elements can be justified. Otherwise, for a slight improvement in terms of capacity, enlarging the system size (hence increasing its complexity) is worthless. 


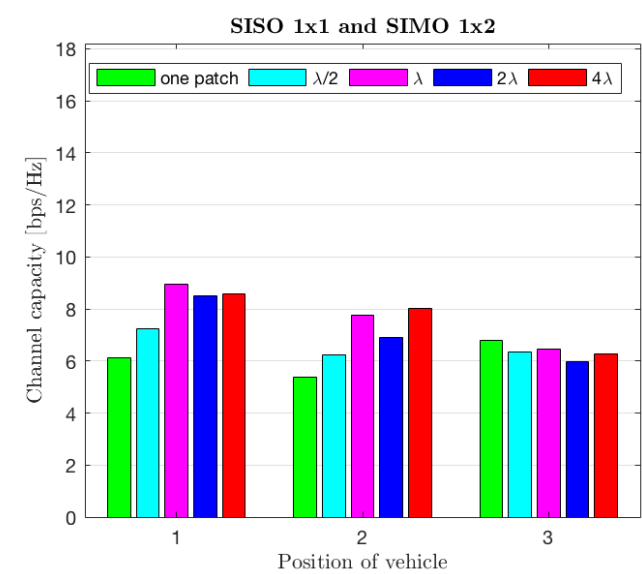

(a)

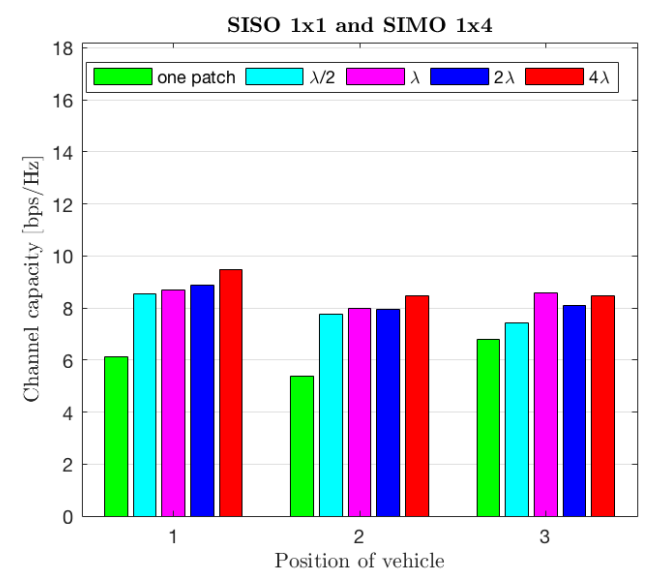

(b)

Fig. 6. Channel capacity at each car position for SISO and SIMO

\section{Results}

1) Massive SISO: The first setup to be analyzed, which will be used later as a reference, is composed of a massive 64-element BS using beamforming to transmit to a single patch antenna in the car. The BS radiation pattern will point to the different car positions $\left(P_{1}, P_{2}, P_{3}\right)$, being more directive when the target is close to the broadside direction, while the car always keeps the same pattern, but moves its position and modifies its orientation to follow the road.

In this case, transmitter power is focused on the car, with a certain footprint around the central point that takes advantage of the BS gain. Otherwise, only one antenna is used as a receiver. Fig. 6 shows the capacity compared with two SIMO systems (1x2 and 1x4).

2) Massive SIMO 1x2 and 1x4: Fig. 6a depicts the capacity for SISO and SIMO $1 \times 2$ for all positions of the vehicle. An increment in capacity can be observed at car position 1 and 2 by introducing a second patch antenna. At position 3 the performance is almost equal in all cases. Fig. $6 \mathrm{~b}$ illustrates that, upon introducing four elements on the car rooftop, the capacity increases as well. For all distances, an improvement in

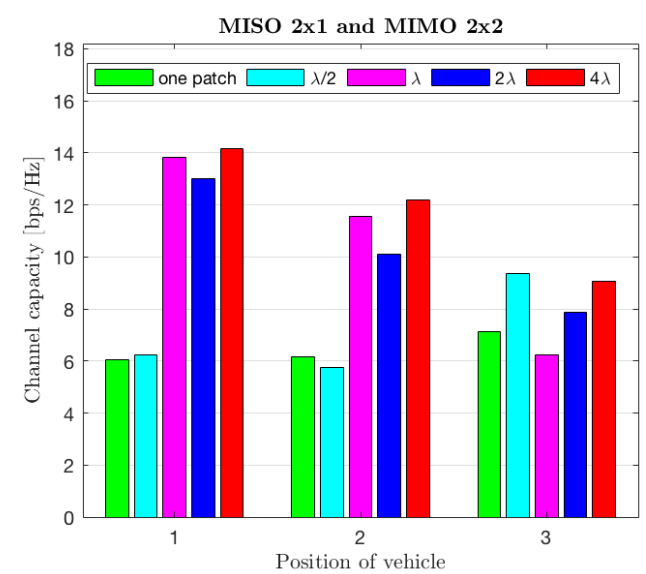

(a)

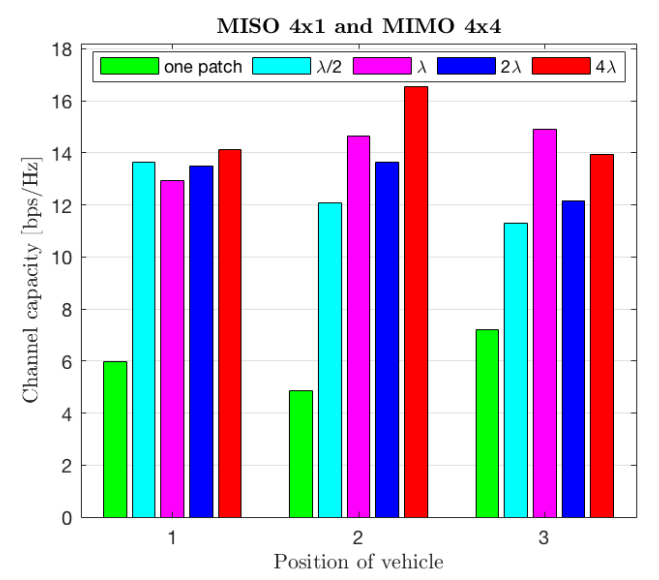

(b)

Fig. 7. Channel capacity at each car position for MIMO

terms of capacity can be observed, even for the third position, in comparison with using only two elements.

3) Massive MIMO $2 \times 2$ and 4x4: Fig. 7a shows the capacity for MISO 2x1 and MIMO 2x2 for all positions of the vehicle. Here, an improvement on capacity is observed when the interelement spacing is greater. Similar to Fig. 6a, all values are reduced for position 3 .

Fig. $7 b$ depicts the capacity for MISO $4 \times 1$ and MIMO $4 \times 4$. The MIMO $4 \times 4$ case provides the highest channel capacity. This can also be seen specially at position 3 . In this case, the improvement is very clear with respect to the MIMO $2 \times 2$ configuration.

\section{Eigenvalues and inter-element spacing}

The relationship between the eigenvalues and the interelement spacing is studied as well. In Fig. 5, the magnitudes of the eigenvalues change for the two distances specified in the graphs. Otherwise, it is observed that MIMO $4 \times 4$ follows a similar evolution, with more relevant eigenvalues at position 2. Regarding MIMO 2x2, the eigenvalues improve significantly at position 1 and 2 if the distance is $4 \lambda$. When the spacing between elements is smaller, the correlation between 
cross terms of the channel matrix should be larger and it implies that eigenvalues are more heterogeneous. The general behavior of the results seems to combine a general tendency consistent with MIMO principle that increases eigenvalues and capacities with inter-element distances. The specific behavior also depends on the amount of elements in the car, which seems to indicate that with higher number of elements, larger inter-element distance is necessary to obtain the optimal performance. A larger separation may be needed to see a real improvement because there are more elements in the same space and the influence of the rest at each input is more noticeable. In any case, capacity is slightly increasing when passing from order 2 to 4 , but might not be enough to justify the extra antennas. Here further simulations have to be performed with larger inter-element spacing on the top of the vehicle.

\section{CONCLUSION}

In our work, we have numerically analyzed the impact on the eigenvalues and consequently on the channel capacity, by comparing different MIMO modalities with distinct inter element spacing. A system model has been proposed for simulations based analysis. The initial results show a gain in terms of channel capacity by increasing the inter element spacing of the antenna setup on the rooftop of the vehicle. Here further research is needed and experimental measurements is

expected to be done for the conference presentation.

\section{ACKNOWLEDGMENT}

This work was supported by the Spanish "Comision Interministerial de Ciencia y Tecnologia" (CICYT) under projects TEC2013-47360-C3-1-P, TEC2016-78028-C3-1-P, MDM2016-O6OO and Altair Engineering, Inc.

\section{REFERENCES}

[1] European Automobile Manufacturers' Association (ACEA), Frequency Bands for V2X, ACEA Position Paper, Dec. 2016.

[2] K. Abboud, H. A. Omar and W. Zhuang, Interworking of DSRC and Cellular Network Technologies for V2X Communications: A Survey, IEEE Transactions on Vehicular Technology, Vol. 65, Num. 12, Dec. 2016.

[3] Altair Engineering,"WinProp Software for Wave Propagation and Radio Planning" http://www.altairhyperworks.com/product/FEKO/WinPropPropagation-Modeling/. Accessed October $10^{\text {th }}, 2017$.

[4] Altair HyperWorks Version 2017, FEKO User Manual, Jan. 2017.

[5] G. J. Foschini and M. J. Gans. On Limits of Wireless Communications in a Fading Environment when Using Multiple Antennas, Wireless Personal Commun., vol. 6, no. 3, pp. 311-335. Mar. 1998.

[6] M. Almarashli and S. Lindenmeier, A New Method for Evaluation of LTE MIMO Antennas in Automotive Application, $11^{\text {th }}$ European Conference on Antennas and Propagation (EUCAP), 2017. 


\section{A.3 Conference Paper: ADAPTIVE 2018}

The conference paper with the title: "Vehicle Antenna Footprint Optimization for Efficient MIMO V2X Communications"; was published and presented at the 10th Internatinal Conference on Adaptive and Self-Adaptive Systems and Applications at Barcelona, Spain. 


\title{
Vehicle Antenna Footprint Optimization for Efficient MIMO V2X Communications
}

\author{
Andreas Pfadler, Christian Ballesteros, Jordi Romeu, and Lluis Jofre \\ Antenna Laboratory \\ Signal Theory and Communications Department \\ Universitat Politecnica de Catalunya \\ Barcelona, Spain \\ Email: \{andreas.pfadler, christian.ballesteros, romeu, jofre\}@tsc.upc.edu
}

\begin{abstract}
Improving efficiency of upcoming vehicle communication networks is one of the main goals in near-future wireless systems. In addition, multi-antenna configurations are known as the main technique to improve the system performance with current constrains, such as the limited spectrum. They are an unlimited and non-lasting resource, but they imply a more complex implementation. In this context, the design of this type of geometries must be optimized considering the entire scenario and the final propagation conditions. Due to the relation between the propagation environment and the correlation between elements, the antenna inter-element spacing has to be adjusted to reach the maximum performance at the minimum possible footprint size. This work investigates the impact of correlation on channel capacity and also proposes a proper separation of the elements when they are mounted on a vehicle for two different urban scenarios: communication between two vehicles (V2V) and car connected to the cellular network (V2I).
\end{abstract}

Keywords-MIMO systems, Urban propagation, Vehicles.

\section{INTRODUCTION}

Vehicle communications have become a recurring topic over the last years due to the increasing interest on creating an efficient and reliable network of connected cars, Vehicle to Everything (V2X). The goal ranges from providing driving aids to the user to self-driving cars. Then, energy efficiency and low latency are two main factors to consider.

In the following work, the focus will be put on the car antenna configuration in order to improve the whole performance of the system by means of numerical simulations for two main situations: Vehicle to Vehicle (V2V), in which two cars try to communicate, and Vehicle to Infrastructure (V2I), where a Base Station (BS) is introduced. For both cases, Multiple Input Multiple Output (MIMO) geometries will be compared with respect to the Single Input Single Output (SISO) case, especially focusing on the impact of the inter-element spacing on the system performance [1]. Conventional MIMO systems for automotive applications are already detailed in [2]. Otherwise, related work in [3] also analyzed the beamforming capabilities of such structures using monopole arrays.

In particular, the study is based in the experimental validation of the specified configurations in a simulated urban environment, in which the cars and the BS will be placed to emulate a realistic situation. A district of the city of Barcelona has been chosen to provide an approach close to reality. The operating frequency is located in the upper side of the S-band, from $3.4 \mathrm{GHz}$ to $3.8 \mathrm{GHz}$, with better propagation properties as compared to higher bands, and which the automotive industry has also become interested in [4].

Next sections are organized as follows: Section II introduces some theoretical concepts that will be useful for the following discussion, Section III describes the environment and numerical tools, Section IV defines the methodology used to evaluate the results, Section V presents the results for the V2I simulations, whereas Section VI does the same for the case of V2V, and, finally, Section VII summarizes the previous work in some major statements deduced from the study.

\section{THEORETICAL BACKGROUND}

\section{A. Capacity in MIMO Channels}

One of the most used figures of merit at physical layer in the analysis of communications systems is the channel capacity. For a MxN MIMO system, being $\mathrm{M}$ the number of transmitting units and $\mathrm{N}$ the receiving ones, capacity may be obtained as [5]:

$$
C=\log _{2}\left(\operatorname{det}\left[\boldsymbol{I}_{N}+\frac{P_{T} \boldsymbol{H} \boldsymbol{H}^{*}}{P_{N}}\right]\right),
$$

where $I_{N}$ is the identity matrix, $\mathbf{H}$ is the channel matrix, whose entries correspond to the addition of all multipaths between each input and output port, $P_{T}$ is the transmission power and $P_{N}$, the noise level. The operator (.)* denotes the conjugate transpose operator, i.e., Hermitian matrix.

$$
\begin{gathered}
\|\boldsymbol{H}\|_{\mathrm{F}}=\left(\sum_{i, j=1}^{N, M}\left|h_{i j}\right|^{2}\right)^{1 / 2} \\
\boldsymbol{H}_{\boldsymbol{C}}=\frac{\boldsymbol{H} \boldsymbol{H}^{*}}{\|\boldsymbol{H}\|_{F}^{2}}
\end{gathered}
$$

Otherwise, we can express the same equation as a function of the channel eigenvalues, $\lambda_{i}\left(\boldsymbol{H}_{\boldsymbol{C}}\right)$. In this case, the channel matrix is normalized using the Frobenius norm, in (2), and the eigenvalues are those corresponding to the product between both $\mathbf{H}$ and its Hermitian, as in (3).

$$
C=\sum_{i=1}^{N} \log _{2}\left(1+\frac{P_{R} \lambda_{i}\left(\boldsymbol{H}_{\boldsymbol{C}}\right)}{P_{N}}\right)
$$

In (4), capacity is defined by means of two main concepts in multi-element cellular communications: the Signal to Noise Ratio (SNR) at the receiver, defined as the ratio between 


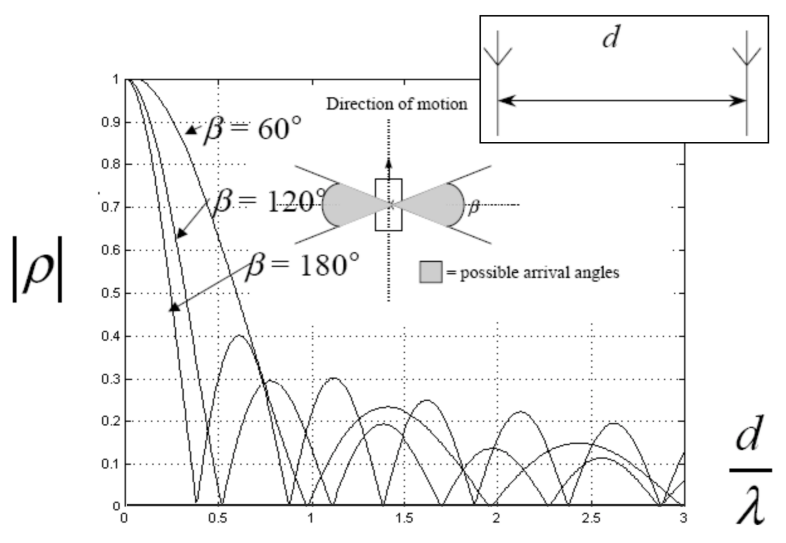

Figure 1. Correlation for two dipoles with changing inter-element spacing and three distinct angle of arrival [7].

received power and noise level $\left(S N R_{R X}=P_{R} / P_{N}\right)$, and the channel richness, determined by the number of relevant eigenvalues. In this analysis, multi-user interference is not considered, but it would affect the noise term (if it is assumed uncorrelated with respect to desired signal), degrading the system performance.

\section{B. Correlation and Spatial Diversity}

In a MIMO system, the distance between antennas has a direct impact on the channel capacity through the correlation between the antenna elements. This fading correlation was researched by Shiu et al. [6]. The smaller the angle spread becomes, the higher is the correlation and the lower the responding channel capacity [5]. Therefore, a higher interelement spacing is needed for small angle spreads.

Figure 1 shows the correlation for three different kind of possible arrival angles for two dipoles with varying interelement spacing. Furthermore, mutual coupling between the antennas is another penalty on the channel capacity, which is not studied in this work.

\section{Simulation ENVIRONMENT}

The study of vehicle communications implies complex and large environments, which require the support of numerical tools to model the performance of the system in a realistic situation. This section is dedicated to detail the elements involved in the process of design and simulation of both the scenario and the antennas. The latter is considered to be the entire vehicle when mounted on the car due to its impact in the field distribution.

\section{A. Software simulation}

Initially, FEKO [8] is used to model the vehicle, as well as the BS for the V2I case, together with the antenna structure. It considers the effect of all the structure when calculating the field. The resulting radiation pattern is imported in a second tool, WinProp [9], that is used to simulate the propagation environment. It is possible to create the scenario for the case of study, with a geometrical approximation of buildings, trees and any other element and simulate using the ray tracing method the interaction of all them when one or more radiators are activated. In this case, a car (V2V) or a BS (V2I) are used as transmitting elements and several points over a virtual

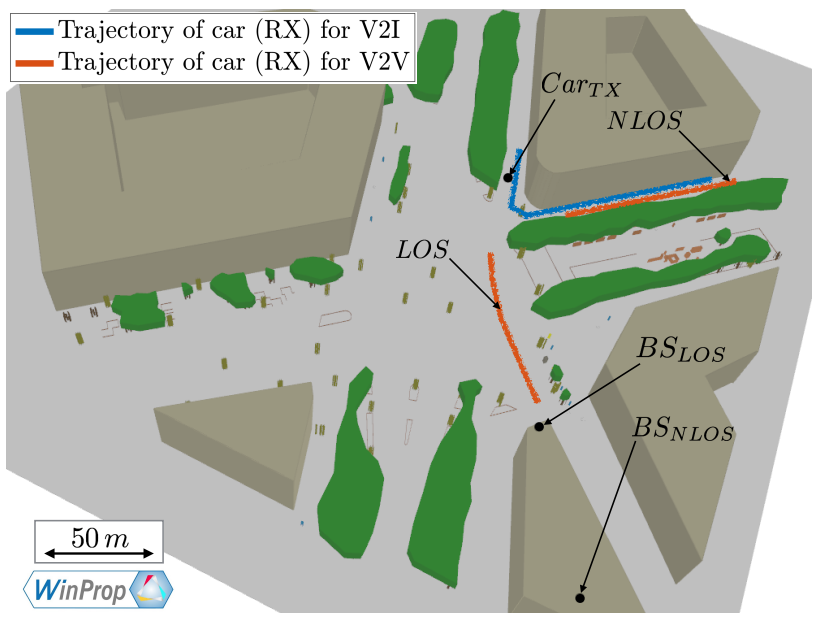

Figure 2. Scenario representation with the location of transmitting vehicle $(\mathrm{V} 2 \mathrm{~V})$, transmitting BS (V2I) and trajectories used in the simulations.

trajectory are assumed to calculate the received fields. At the receiving points, the effect of the car is considered, as the radiation pattern calculation is including its structure. It is important to mention that all receiving points assume a still vehicle located on them, which is neglecting any Doppler effect.

\section{B. Scenario}

The chosen scenario is an urban area with medium-height buildings, some trees and streetlights. In particular, it is a model of an intersection in a district of Barcelona called as L'Eixample, which is known for its rectangular shape and corners close to $90 \mathrm{deg}$. In Figure 2, the 3-D view of the scenario is shown.

All buildings are made of concrete walls of $30 \mathrm{~cm}$ thickness and $18 \mathrm{~m}$ height, some of them including a courtyard. The ground is made of asphalt, streetlights are modelled as metallic cylinders and trees include a solid wooden trunk and the top is assumed to be a foliage semi-transparent to the rays (only a certain attenuation applied as they pass through).

\section{Vehicle configuration}

For the vehicle model, a prototype of a conventional car is used. It includes two types of materials: metal for the body and laminated glass for the windows. Wheels and internal elements are excluded to simplify the design and to reduce computational complexity and time consumption during the simulations.

As shown in Figure $3 \mathrm{a}$, the antenna is mounted on the car rooftop. For V2V communications, both transmitter and receiver will have the same height and, for the case of V2I, if large distances are assumed, the angle of arrival is close to the horizontal plane. In consequence, the ideal radiation pattern should be maximum for angles close to the horizontal plane and minimum in the vertical axis. Thus, the chosen antenna is a monopole. In case of MIMO configurations, two or four antennas are placed in a straight line, appropriated to fit in a shark-fin footprint. 


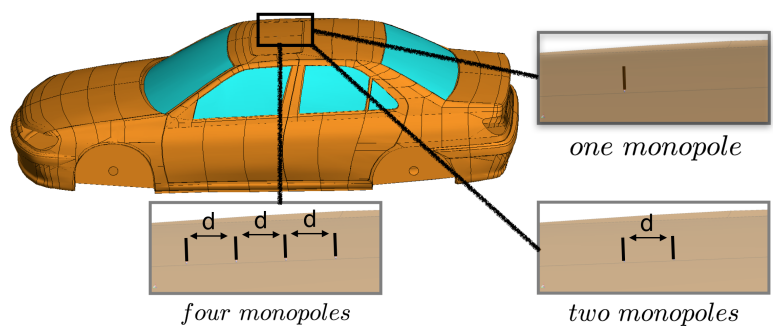

(a) Antenna configurations on the car.

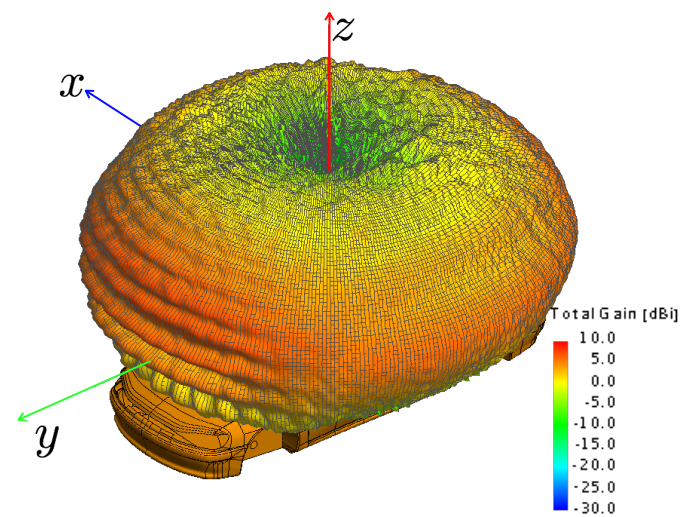

(b) Radiation pattern of a monopole mounted on the car rooftop.

Figure 3. Model of the vehicle and antennas.

\section{Base Station}

It consists of a set of omnidirectional elements placed on the top of a building as shown in Figure 2. The total height is $21 \mathrm{~m}$ over the ground, or $3 \mathrm{~m}$ over the rooftop. Depending on the case, one, two, or four antennas are used (for SISO and MIMO $2 \times 2$ or $4 \times 4$, respectively), with a constant spacing in all cases.

\section{Methodology}

The figure of merit to estimate the system performance is the average capacity for the set of points used in each specific path. For each one, the capacity is calculated using (4), once the channel matrix is obtained by the numerical simulation. The noise level is not fixed in the calculation, but a mean SNR is assumed for the overall trajectory. Its value is set to $10 \mathrm{~dB}$. In addition, in order to determine the achievable performance and validate the results, a theoretical maximum is calculated with ideal channel eigenvalues, i.e., $\sigma_{i}=0.5(\mathrm{i}=1,2)$ for MIMO $2 \times 2$ and $\sigma_{j}=0.25(\mathrm{j}=1,2,3,4)$, for MIMO $4 \times 4$.

The spacing between the antenna elements (monopoles) on top of the car is chosen to be in the range of 0.1 to 4 times the wavelength, in steps of $0.1 \lambda$. For each one, the average capacity over a given trajectory is calculated and then compared with respect to the SISO case.

\section{IMPACT OF THE INTER-ELEMENT SPACING IN V2I MIMO COMMUNICATIONS}

In this section, the performance in terms of capacity is studied for MIMO V2I systems. The analysis distinguishes between two different situations: Line of Sight (LOS) and Non Line of Sight (NLOS). In the first case, the BS is placed at the edge of the building, whereas, in the latter, the direct view is blocked by placing it some meters backwards (see Figure 2).

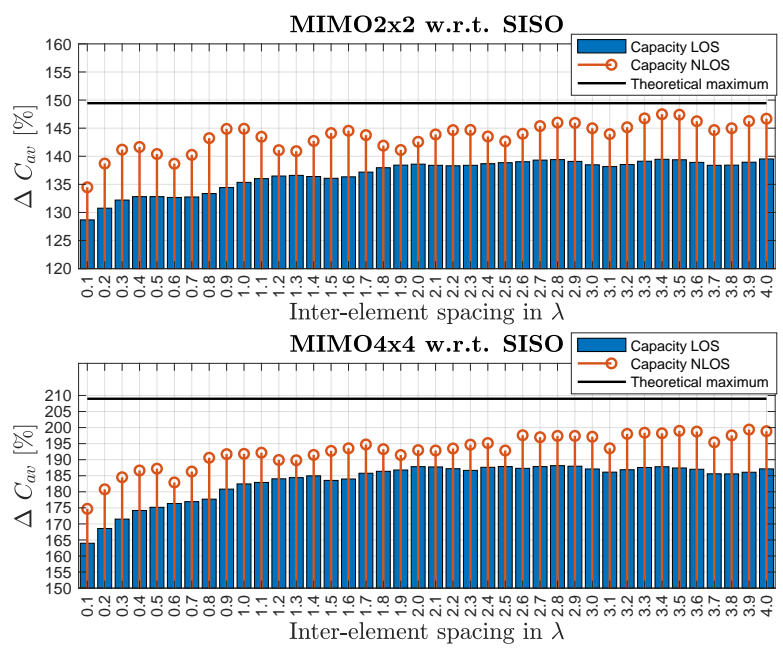

Figure 4. V2I channel capacity for different inter-element spacing for MIMO $2 \times 2$ and MIMO $4 \times 4$ with respect to the SISO channel capacity in percent and a fixed SNR of $10 \mathrm{~dB}$.

Figure 4 shows the average channel capacity obtained for distinct inter-element spacing with respect to the SISO case in percentage. It illustrates the MIMO 2x2 case (upper figure) for the LOS in blue, the NLOS in red and the theoretical maximum in black. The same illustration is shown in the lower figure for MIMO 4x4.

First, if MIMO $2 \times 2$ is analyzed, it is observed a theoretical maximum channel capacity $149 \%$ respect to SISO. The minimum for LOS case is $129 \%$ and, in NLOS, it is $135 \%$, both obtained with an inter-element spacing of 0.1 wavelength. The maximum with $147 \%$ for NLOS is achieved with a spacing of $3.4 \lambda$ and is almost reaching the theoretical maximum of $149 \%$. In the case of LOS, the maximum of $139 \%$ although the channel capacity is almost flat after interelement distances of $1.9 \lambda$. The evolution for NLOS is expected as in the theoretical graph (see Figure 1). Regarding MIMO $4 \times 4$, the theoretical maximum channel capacity is now $209 \%$. The lowest channel capacity is again obtained for both LOS and NLOS when inter-element spacing is 0.1 wavelengths ( $164 \%$ and $175 \%$, respectively). On the other hand, the greatest capacity is achieved at the spacing of $2.8 \lambda$, with $188 \%$, for LOS and $3.9 \lambda$, and $198 \%$ for NLOS.

From the previous results, it is deduced that MIMO $2 \times 2$ is almost reaching the theoretical maximum for NLOS communication, whereas MIMO $4 \times 4$ has a bigger step until its maximum. It can be determined that the channel is not rich enough, even increasing the inter-element spacing. Otherwise, it is also true that MIMO $4 \times 4$ provides a larger improvement with respect to SISO in terms of capacity although the channel rank is low.

Additionally, in MIMO 4x4 case, there is a more significant increment when the spacing is increased if the values are compared from the lowest distances to the largest. As stated in [10], "the specific behavior is also depending on the amount of elements in the car, which seems to indicate that higher is the number of elements, higher may be the necessary inter-element distance to obtain the optimal performance." 


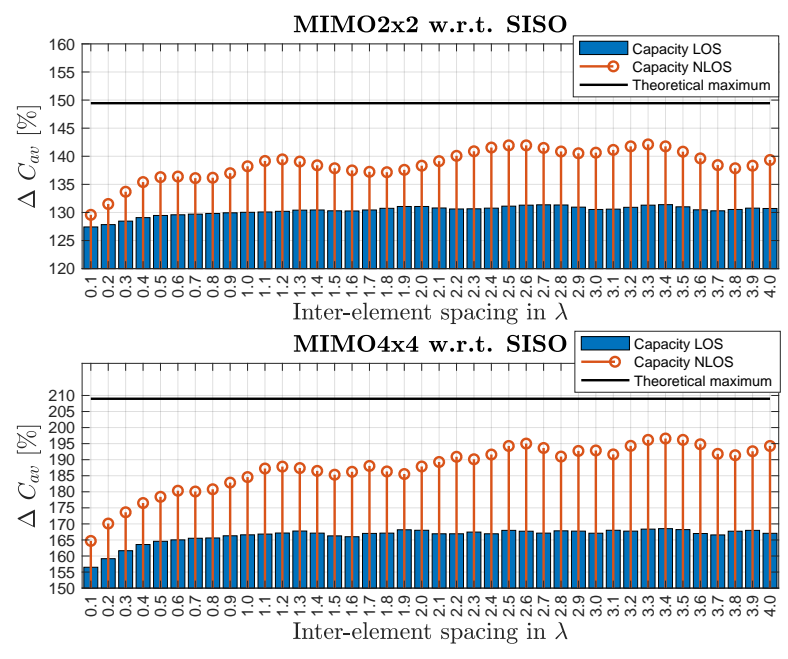

Figure 5. V2V channel capacity for different inter-element spacing for MIMO $2 \times 2$ and MIMO $4 \times 4$ with respect to the SISO channel capacity in percent and a fixed SNR of $10 \mathrm{~dB}$.

\section{IMPACT OF INTER-ELEMENT SPACING IN V2V MIMO COMMUNICATIONS}

The goal now is to analyze the communication between two equal vehicles (V2V). This situation has some major differences as compared to previous case: both transmitter and receiver have the same height, their spacing is modified at the same time and both use monopole antennas.

The transmitting vehicle is fixed in a still position. Otherwise, two trajectories are considered for the receiver: one in LOS in which the car is driving along the same path as the transmitter and another in NLOS behind the building close to the corner (Figure 2).

Figure 5 compares both situations when MIMO 2x2 or $4 \times 4$ are used. In contrast with V2I model, the increment in capacity is much more evident for NLOS. It is necessary to remind at this point that the SNR is fixed to $10 \mathrm{~dB}$ and the performance is only affected by the eigenvalues distribution. Then, we can deduce that the rank of the channel matrix is clearly increasing when there is no direct path.

In NLOS, the average capacity is very similar to previous case. There is a maximum of $123 \%$ at $3.3 \lambda$ for MIMO $2 \times 2$ and $194 \%$ at $3.4 \lambda$ for MIMO $4 \times 4$. In any case, the oscillation is considerably stable above $2 \lambda$. Otherwise, for the LOS situation, capacity is much more reduced. This behavior indicates a very strong path, corresponding to the direct view, and low power reflections. Now, the behavior is almost stable after $0.5 \lambda$ and maximum values are $132 \%$ and $168 \%$ for MIMO 2x2 and 4x4, respectively.

\section{CONCLUSIONS}

Based on the realistic numerical modeling of a V2X urban environment, two main conclusions may be extracted. In terms of antenna footprint, it has been shown that higher the order of the MIMO system, larger has to be the inter-element distance to obtain optimal performance. Furthermore, the increment of the distance improves capacity in both LOS and NLOS for $\mathrm{V} 2 \mathrm{I}$; whereas, for $\mathrm{V} 2 \mathrm{~V}$, a significant improvement is only obtained in NLOS.

\section{ACKNOWLEDGMENT}

This work was supported by the Spanish "Comision Interministerial de Ciencia y Tecnologia" (CICYT) under projects TEC2013-47360-C3-1-P, TEC2016-78028-C3-1-P, MDM2016-O6OO and Altair Engineering, Inc.

\section{REFERENCES}

[1] C.-N. Chuah, D. N. C. Tse, J. M. Kahn, and R. A. Valenzuela, "Capacity scaling in mimo wireless systems under correlated fading," IEEE Transactions on Information Theory, vol. 48, no. 3, Mar 2002, pp. 637-650.

[2] A. Paier, L. Bernado, J. Karedal, O. Klemp, and A. Kwoczek, "Overview of vehicle-to-vehicle radio channel measurements for collision avoidance applications," in 2010 IEEE 71st Vehicular Technology Conference, May 2010, pp. 1-5.

[3] T. Kopacz, A. Narbudowicz, D. Heberling, and M. J. Ammann, "Evaluation of automotive mimo antennas for $\mathrm{v} 2 \mathrm{v}$ communication in urban intersection scenarios," in Antennas and Propagation (EUCAP), 2017 11th European Conference on. IEEE, 2017, pp. 2907-2911.

[4] "Frequency bands for v2x," ACEA Position Paper, no. 8, 2016.

[5] G. J. Foschini and M. J. Gans, "On limits of wireless communications in a fading environment when using multiple antennas," Wireless personal communications, vol. 6, no. 3, 1998, pp. 311-335.

[6] D. S. Shiu, G. J. Foschini, M. J. Gans, and J. M. Kahn, "Fading correlation and its effect on the capacity of multielement antenna systems," IEEE Transactions on communications, vol. 48, no. 3, 2000, pp. 502-513.

[7] W. C. Jakes and D. C. Cox, Eds., Microwave Mobile Communications. Wiley-IEEE Press, 1994.

[8] Altair Hyperworks, FEKO User Manual 2017, Altair Hyperworks.

[9] Altair Engineering Inc., WinProp Software for Wave Propagation and Radio Planning, Altair Engineering Inc.

[10] A. Pfadler, C. Ballesteros, J. Romeu, and L. Jofre, "Multi-antenna configuration modeling for massive mimo v2i," in Antennas and Propagation (EUCAP), 2018 12th European Conference on. IEEE, 2018, pp. 215-219. 


\section{A.4 Submitted Journal Paper: Special Issue}

The journal paper with the title: "Propagation-based Performance of Massive MIMO Antenna Configurations for V2I Urban Communications"; was submitted to the IEEE Transactions on Antennas and Propagation. 


\title{
Propagation-based Performance of Massive MIMO Antenna Configurations for V2I Urban Communications
}

\author{
A. Pfadler, C. Ballesteros, J. Romeu, Fellow, IEEE, and L. Jofre, Fellow, IEEE
}

\begin{abstract}
Vehicle communications are now emerging towards 5G. Larger data rates than in nowadays LTE systems are required. Therefore, there is a growing research interest in massive MIMO antenna systems due to their higher channel capacity and spectral and energy efficiency compared to conventional MIMO. In the mid-term context, conventional MIMO architectures need to be further optimized for Vehicle to Infrastructure (V2I) communications, since the evolution towards $5 \mathrm{G}$ is progressively advancing and intermediate solutions are essential. In order to study the impact of different multi-antenna geometries and MIMO architectures in both vehicle and Base Station (BS), a quality modeling of a realistic scenario is required. The analysis is based on a numerical simulation of an urban scenario, emulating a portion of the city of Barcelona, comprising a massive MIMO BS and a car with different multi-antenna rooftop solutions and at various positions on a reference trajectory. Several configurations are compared by means of the channel eigenvalues and the corresponding capacities. On top of the vehicle one, two or four monopole antennas are used and the BS is modeled with one, two or four patch antennas when using conventional MIMO and 64 patch antennas grouped as one, two or four beams for massive MIMO.
\end{abstract}

Index Terms-MIMO systems, Antenna arrays, Urban propagation, Vehicles.

\section{INTRODUCTION}

During the last ten years automotive and telecommunications industry have joint efforts on V2I applications due to the increasing interest of users and manufacturers in connecting vehicles to a high-speed network to provide multimedia services and driving assistance. To respond to these demands with the always limited spectrum availability, Multiple Input Multiple Output (MIMO) in general, and massive MIMO in particular, for the BS may introduce significant improvements. The throughput of the system [1] can be significantly enhanced, and multi-user interference reduced [2]. Due to its higher directivity, massive architectures are worthwhile if the

This work was supported by the Spanish "Comision Interministerial de Ciencia y Tecnologia" (CICYT) under projects TEC2013-47360-C3-1-P, TEC2016-78028-C3-1-P, MDM2016-O6OO and Altair Engineering, Inc.

A. Pfadler and C. Ballesteros are graduate student members of the AntennaLab research group inside the Signal Theory and Communication Department of the "Universitat Politecnica de Catalunya" (UPC), Barcelona, Spain. (e-mail: \{andreas.pfadler, christian.ballesteros\}@ tsc.upc.edu)

Dr. L. Jofre and Dr. J. Romeu are full professors at the UPC and members of the Antenna Laboratory (AntennaLab). They have authored more than a hundred of scientific and technical papers, reports, and chapters in specialized volumes antennas, electromagnetic scattering and imaging, system miniaturization for wireless, and sensing industrial and bio-applications. (email: \{jofre, romeu\}@tsc.upc.edu)

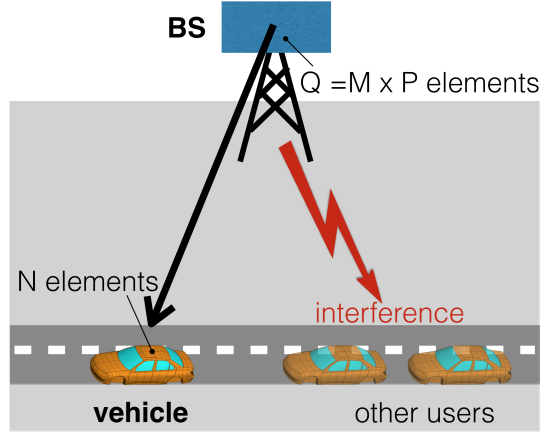

Fig. 1. Generic model of a V2I scenario.

user location is known and the update of the steered beam is fast enough.

Fig. 1 illustrates a generic model of a V2I communication system. The general operation of the entire system would consist on a first step, in which the potential users send a pilot signal that allows the BS to identify and locate them spatially. With this information, in a second stage, the BS radiates into the coverage area with a focusing strategy depending on the specific configuration. It illuminates the different areas where the users (vehicles) are located. Due to the angular distribution of the BS field, some other regions may be reached by the signal and an interference level may be noticed outside the area of interest.

Table I shows the overview of all investigated modalities. It is distinguished between conventional $M \times N$ MIMO, where $M$ refers to the number of BS transmitting units and $N$, to the vehicle receiving ones, and massive configurations, where a total amount of $\mathrm{Q}$ elements are separated in a set of $\mathrm{M}$ modules of $\mathrm{P}$ elements each $(Q=M \times P)$.

The Multi-user massive Single Input Single Output (MU$\mathrm{mSISO}$ ) BS is composed by Q antenna elements with steering capabilities (one beam towards the receiver). It can be seen as a Single Input Single Output (SISO) communication with beamforming at the BS. MU-mSISO is normally called massive MIMO in literature, as defined in [2] and it is different from conventional MIMO techniques [3]. Subdividing the massive BS into M modules instead of working as a single antenna, the Multi-user massive Multiple Input Multiple Output (MUmMIMO) configurations allows to introduce $\mathrm{M}$ ports feeding a set of $\mathrm{P}$ antennas each. Therefore, the channel matrix $\mathbf{H}$ consists of $M \times N$ entries. $\mathrm{M}$ beams are steered towards 
TABLE I

OVERVIEW OF ALL INVESTIGATED MIMO MODALITIES WITH $x_{x}$ AS THE BS INPUT AND $y_{x}$ AS THE RECEIVING VEHICLE OUTPUT, AND THE CHANNEL H IN BETWEEN.

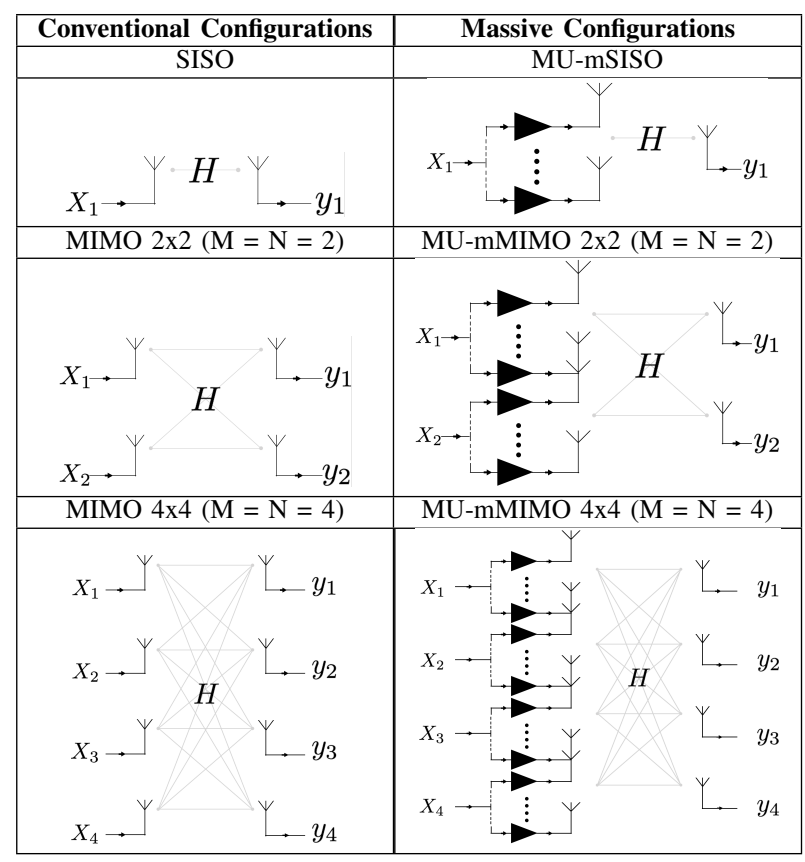

the receiver with $\mathrm{N}$ single antennas, which is similar to the conventional MIMO case but using beamforming.

Regulation entities have defined some standards, with the allocation of spectrum to support the development of new technologies. On one hand, the Electronic Communications Committee (ECC) and the European Union defined a $30 \mathrm{MHz}$ band at $5.9 \mathrm{GHz}$, which was finally integrated in the IEEE 802.11p standard for Vehicle to Everything (V2X) applications [4]. On the other hand, the automotive industry is trying to follow another approach, focusing on lower bands with better propagation properties for mobile communications: $3.4 \mathrm{GHz}$ to $3.8 \mathrm{GHz}$ [5]. In this work, the latter band is chosen to design the model due to better propagation properties.

The analysis will be centered on the physical layer modeling of the propagation environment with different antenna configurations at both the BS and the car for an urban scenario by means of rigorous electromagnetic numerical simulation tools.

The study will consider in particular the:

1) comparison of the energy efficiency when comparing conventional and massive MIMO systems,

2) reduction of inter-user interference, and

3) improvement on channel capacity for different BS modular decomposition.

Next sections are organized as follows. Section II provides a brief description of the MIMO channel. Section III describes the system model that is used in the study. Section IV describes the physical characterization of the channel used in the numerical analysis. In section $\mathrm{V}$, the results of different configurations are presented and discussed. Finally, conclusions are summarized in Section VI.

\section{THE MIMO CHANNEL}

The channel matrix $\mathbf{H}$ describes the relation between the BS (considered as the transmitter) input vector $\boldsymbol{x}(t)$ and the vehicle (considered as the receiver) output vector $\boldsymbol{y}(t)$ :

$$
\boldsymbol{y}(t)=\boldsymbol{H} \boldsymbol{x}(t),
$$

where $\mathbf{H}$ is an NxM matrix for a MIMO MxN system. The channel transfer function for $\mathrm{i}$-th transmitter and $\mathrm{j}$-th receiver is obtained as the addition of all multipath contributions between them and can be expressed as follows:

$$
h_{i j}(\boldsymbol{r})=\sum_{t=1}^{T} \Gamma\left(\boldsymbol{r}_{\boldsymbol{t}}\right) e^{-j \boldsymbol{k} \cdot \boldsymbol{r}_{\boldsymbol{t}}},
$$

where $\Gamma\left(\boldsymbol{r}_{\boldsymbol{t}}\right)$ is the complex envelope of the channel transfer function for the $\boldsymbol{r}_{\boldsymbol{t}}$ multipath distance vector and $k=2 \pi / \lambda$ is the wave number.

From previous definition, the capacity for a MIMO system may be obtained as [6]:

$$
C=\log _{2}\left(\operatorname{det}\left[\boldsymbol{I}_{N}+\frac{P_{T} \boldsymbol{H}^{*}}{P_{N}}\right]\right),
$$

where $P_{T}$ and $P_{N}(-90 \mathrm{dBm})$ are transmit and noise power, respectively, $I_{N}$ is the identity matrix, and (.)* denotes the transpose conjugate.

The channel matrix can be normalized with the Frobenius norm.

$$
\|\boldsymbol{H}\|_{F}=\left(\sum_{i, j=1}^{N, M}\left|h_{i j}\right|^{2}\right)^{1 / 2} .
$$

The channel capacity may be also defined as a function of the Frobenius normalized eigenvalues $\lambda_{i}[6]$ :

$$
C=\sum_{i=1}^{N} \log _{2}\left(1+\frac{P_{R} \lambda_{i}}{P_{N}}\right),
$$

where $P_{R}$ denotes the received power.

\section{SySTEM MODEL}

The system model consists of the urban environment, the BS configuration and the multi-antenna vehicle setup.

\section{A. Urban Environment}

In order to obtain a more realistic approach for the analysis of a car in an urban area, a portion of a real city is chosen to simulate the V2I environment. In particular, Fig. 2 depicts two crossing streets in the city of Barcelona, corresponding to the district of L'Eixample (characterized by its rectangular shapes and almost 90 degrees corners). The environment is composed of a ground plane made of asphalt, five buildings, three of them with courtyards, traffic lights and trees. The height of all buildings is $18 \mathrm{~m}$ and the BS is placed on the top of one building at height of $21 \mathrm{~m}$ from the ground, i.e. $3 \mathrm{~m}$ above the rooftop. Object materials are considered into the model and, in this respect, buildings are made of concrete, trees are made of a wooden trunk and vegetation on top, which let the rays to pass through the latter at the cost of additional attenuation, and traffic lights are metallic. 


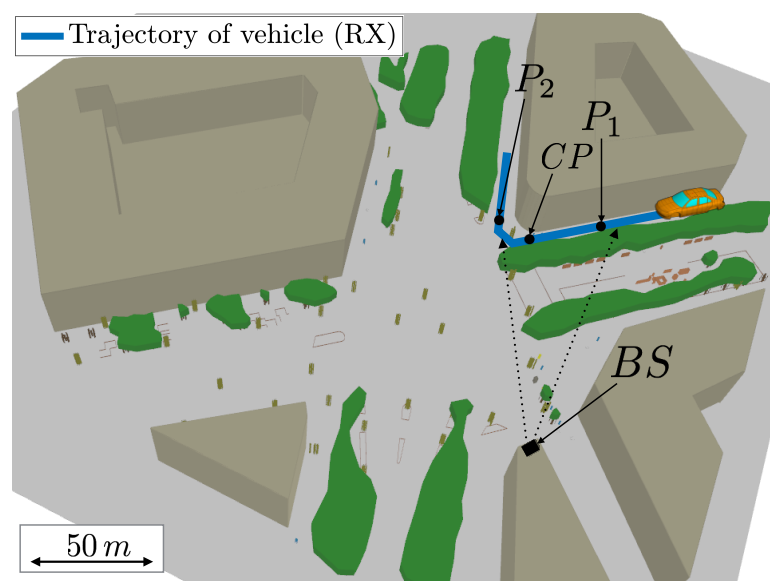

Fig. 2. The scenario of the intersection in Barcelona modeled in WinProp.

As represented in Fig. 2, a reference trajectory (143.4 m long sampled every $0.1 \mathrm{~m}$ ) is defined for the moving car, with three characteristic points: $P_{1}$ (at $45 \mathrm{~m}$ on the trajectory), $P_{2}$ $(85 \mathrm{~m})$ and $\mathrm{CP}(70 \mathrm{~m})$. The BS steers its beam to follow the trajectory in a discrete way, where $P_{1}$ and $P_{2}$ represent the two specific points at which it is pointing for two positions of the car. Otherwise, $\mathrm{CP}$ represents the closest point to the BS and it is located on the corner of the street.

For the simulation, the maximum number of interactions is set to three reflections, two diffractions and one transmission for each ray. In total, up to 20 different paths are taken into account from the transmitter to each receiver position on the trajectory.

\section{B. Base Station Multi-antenna Geometry}

For the BS, two configurations are considered:

- Conventional MIMO with either one, two, or four independent isotropic radiating elements able to deliver the same power over the entire trajectory. The total transmitted power is $10 \mathrm{dBm}$ and it is equally distributed among all the elements.

- A potential 5G massive MIMO geometry with $Q=64$ patch-antenna elements, as proposed by Shintaro Shinjo et al. in [7]. The total transmitted power is now $0 \mathrm{dBm}$ and, as can be seen in Fig. 3, the BS may be organized in three different geometries:

1) a single 64-element module,

2) two modules of 32 elements and half of the power each, and

3) four modules of 16 elements each and a quarter of the power each.

At each module, beamforming is produced with uniform amplitude weights and only the phase is considered in the steering, -Phased Array Antenna (PAA)-. Fig. 4 shows an example of the power map when the BS is steered towards $P_{2}$

At the BS, mutual coupling might be a source of inaccuracy in both the uplink and downlink [8]. It affects the estimation of the car location and the focusing capabilities towards the

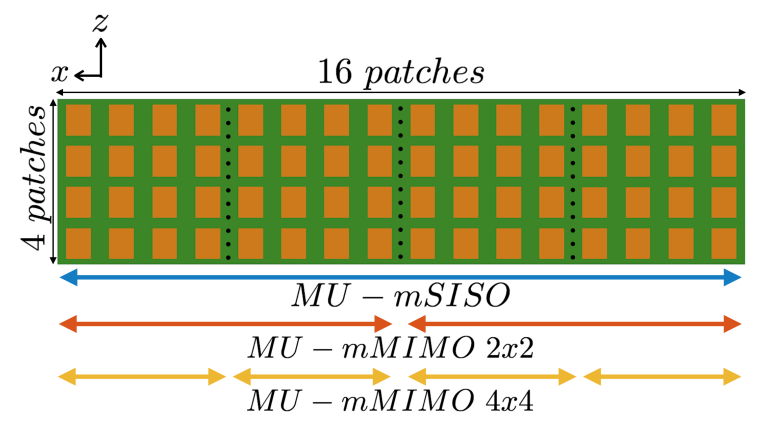

Fig. 3. Massive $B S$ with $4 \times 16(Q=64)$ patch antennas and the subdivision into distinct massive MIMO configurations.

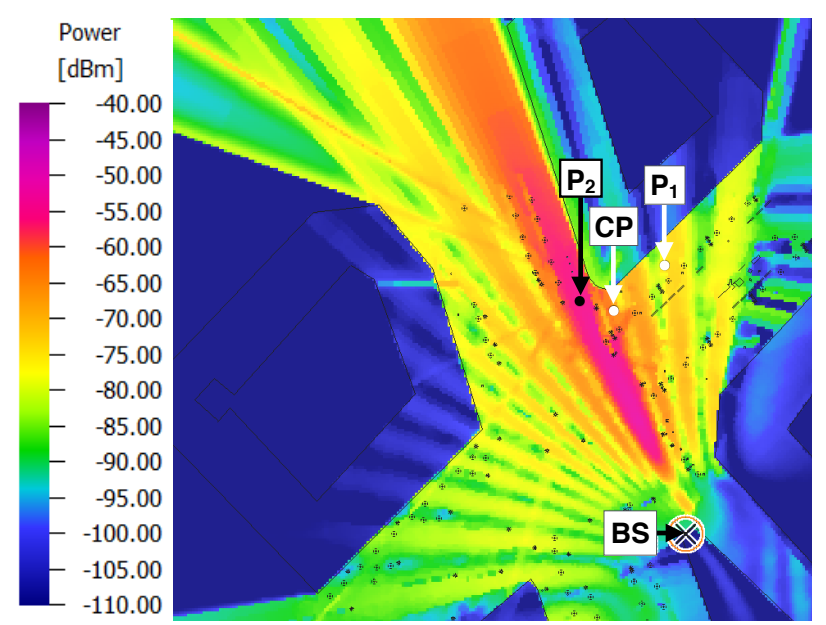

Fig. 4. Power map of the incoherent power received at a height of $1.42 \mathrm{~m}$ for MU-mSISO towards $P_{2}$ with $P_{T}=0 \mathrm{dBm}$ and a resolution of $1 \mathrm{~m}$.

user. In consequence, mutual coupling must be considered in the model. The simulation software already assumes the coupling effect when the field distribution is calculated, but MU-mMIMO modules are simulated separately, thus the effect between them is neglected. In consequence, it is mandatory to calculate the correlation coefficient between those to determine whether the assumption is valid or not.

Following the same steps defined in [9], [10], and [11], it is possible to evaluate the correlation, $\rho_{e}$, between two radiating elements if their field distribution is known. For the modular distribution presented in Fig. 3, numerical models determine that cross-correlation terms are negligible $\left(\rho_{e} \ll 1\right)$ between modules for both MU-mMIMO 2x2 and MU-mMIMO 4x4.

\section{Vehicle Multi-antenna Geometry}

For the vehicle, one, two, or four independent monopole antennas (see Fig. 5) are considered. They are aligned into a shark-fin like structure on the front part of the car rooftop, at $1.42 \mathrm{~m}$ from the ground.

For a proper low correlation [12], an inter-element distance of half wavelength $(\lambda / 2)$ is considered [13]. The radiation pattern of the different elements is calculated taking into account the presence of the whole car. 


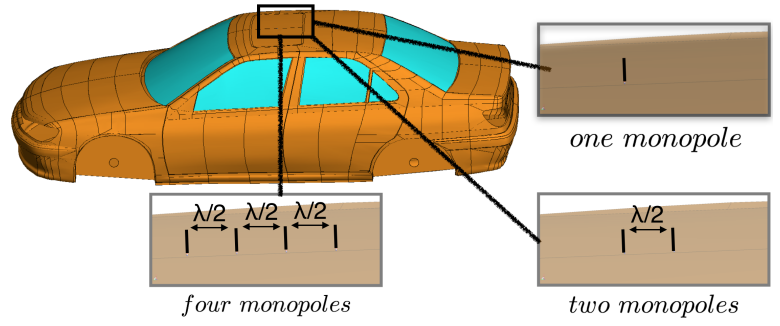

Fig. 5. Three different vehicle antenna setups with one, two, and four monopole antennas at $1.42 \mathrm{~m}$.

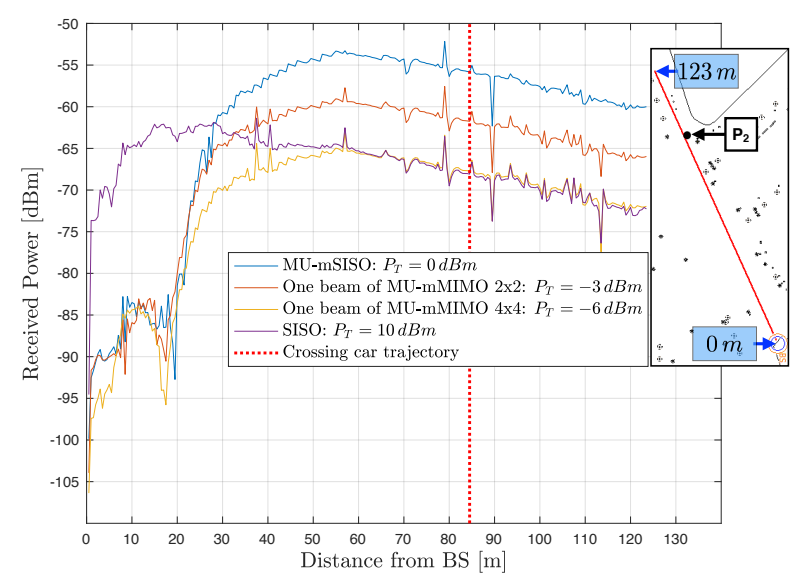

Fig. 6. Received power for massive MIMO towards $P_{2}$ and conventional SISO as a function of distance at a height of $1.42 \mathrm{~m}$.

\section{Physical Multi-Ray Propagation CHANNEL MODEL}

\section{A. Numerical Propagation Model}

The calculation of the different matrix elements, $h_{i j}(\boldsymbol{r})$, considers two main parts: the angular radiation of the BS individual radiating elements (simulated with FEKO) considering the effect of its nearby geometry, i.e. the different elements of each module in case of the BS or the car structure for the user, and the superposition of all radiopaths (given by WinProp) from each transmitting element to each receiving one. From the obtained channel matrix, the eigenvalues and the resulting capacity may be calculated to compare the performance of the different configurations.

\section{B. Validation of Propagation Environment}

The impact of all the objects in the scenario, specially the ground, can be critical in the final distribution of the fields [14]. The propagation software, by means of the ray tracing method, considers the interaction of several points in the environment between the transmitter location and the receiver points considered in the reference trajectory. In order to validate whether it is consistent or not with the study in [14], a straight line in Line of Sight (LOS) condition for all points is assumed, starting at the BS coordinates, but at the same height as the car. The target, in this case, is position $P_{2}$, which is also included in the new set of points.
As can be seen in Fig. 6, the received power after adding coherently the contribution of the multipath depends on the configuration used in each case. The SISO model, in which a single patch antenna is used at the transmitter, follows the expected tendency. The maximum of received power is found some meters after the tranmitter and, from this point, the function decreases monotically with a constant slope of $27 \mathrm{~dB} /$ decade, which agrees with [14]. Otherwise, when the massive BS is introduced, an important difference may be noticed. The maximum is located at a further distance. As it was expected, the beam focusing on the user concentrates the power on a region close to the vehicle. If all elements are used as a single beamformer, which is the case of MU$\mathrm{mSISO}$, the maximum power is larger than in the case of conventional SISO. This behavior denotes the higher efficiency of this type of systems, which are able to deliver more power to the user even with lower consumption, as it is the case. For MU-mMIMO, the behavior is very similar but the usage of $1 / M$ of the total power at each module reduces the received power.

Due to the use of uniform weighting amplitudes, the presence a sidelobe produces a small peak in the first meters. The level of the sidelobe depends on the number of antennas per module, thus this spurious peak is larger as the order of the MIMO increases (less elements to create the beam). In general, the three massive configurations show the same behavior, with different power levels according to their modular distribution.

\section{Simulation Results For V2I MiMO Systems}

In this section, the performance of both conventional and massive MIMO configurations is analyzed for the previously defined simulation environment. Channel capacity and eigenvalues are computed along the reference trajectory (see Fig. 2) A figure of merit for the whole reference trajectory is defined as the average of the capacity at each of the trajectory points:

$$
C_{a v .}=\frac{1}{N_{S}} \sum_{i=1}^{N_{S}} C_{i}
$$

where $N_{S}$ is the number of sampled receiver positions.

\section{A. Conventional Configurations}

Fig. 7 shows the channel capacity for the different conventional configurations (SISO, MIMO 2X2 and MIMO 4X4). The graph indicates, as expected, that the higher is the order of the MIMO system, higher is the achievable capacity. The line corresponding to MIMO $4 \times 4$ case is always above the others, with a clear peak around the corner, where high Signal to Noise Ratio (SNR) exists and even a small contribution of lower eigenvalues represents a noticeable gain in capacity. The same effect is present in the MIMO 2x2 case, which is always better than SISO and improves when approaching the points around the corner.

Fig. 8a illustrates the average capacity. As previously seen for the entire trajectory, it is larger for higher MIMO order architectures, therefore, the channel is rich enough to use a multi-antenna configuration. The relative improvement respect 


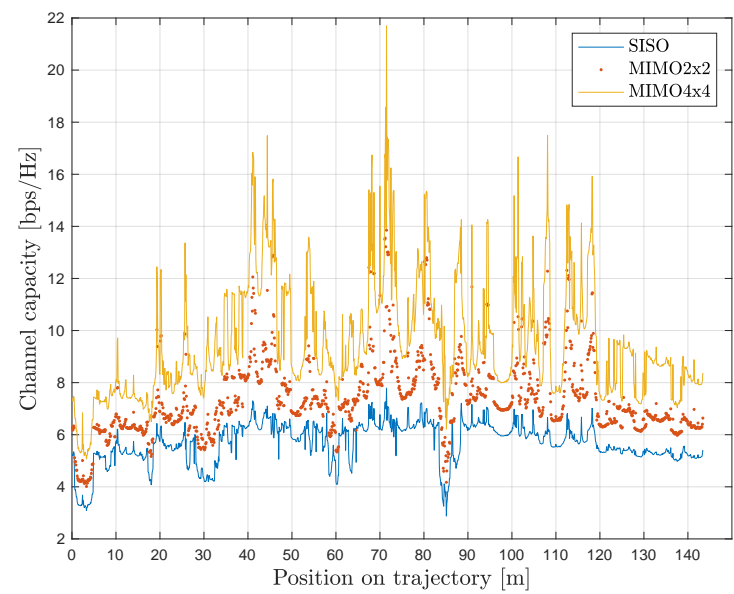

Fig. 7. Channel capacity for conventional MIMO over the entire trajectory.

the SISO, which is shown in Fig. 8b, is almost $30 \%$ for MIMO $2 \times 2$ and $70 \%$, for MIMO $4 \times 4$.

In this case, there is not any improvement in terms of consumed energy because the same amount of power is used for the three configurations. Due to the equally distributed power in all transmitting antennas, the improvement in capacity results from the multipath contributions of the channel.

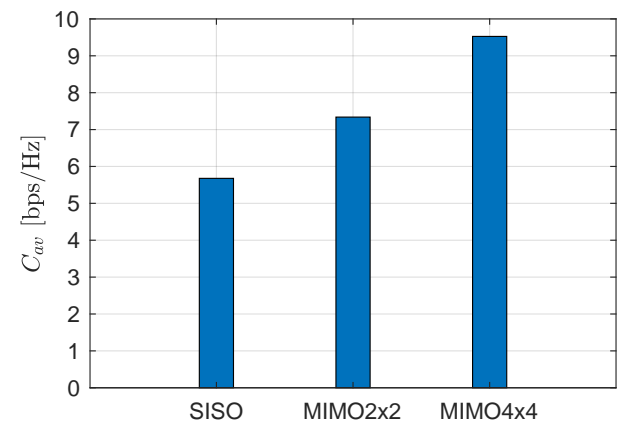

(a) Average channel capacity.

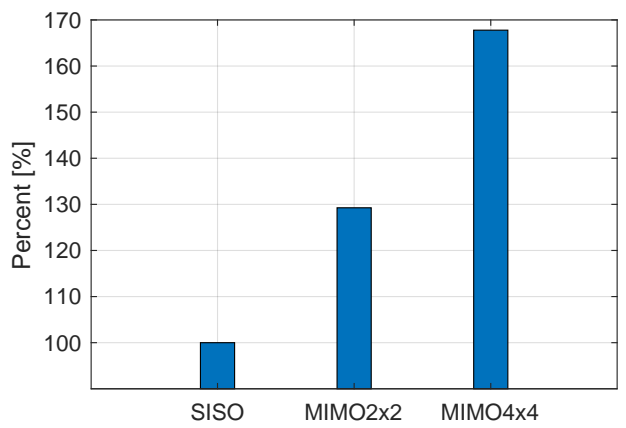

(b) Average capacity in percentage respect to SISO.

Fig. 8. Average channel capacity for conventional MIMO over the entire trajectory (mean SNR of $12 \mathrm{~dB}$ ).

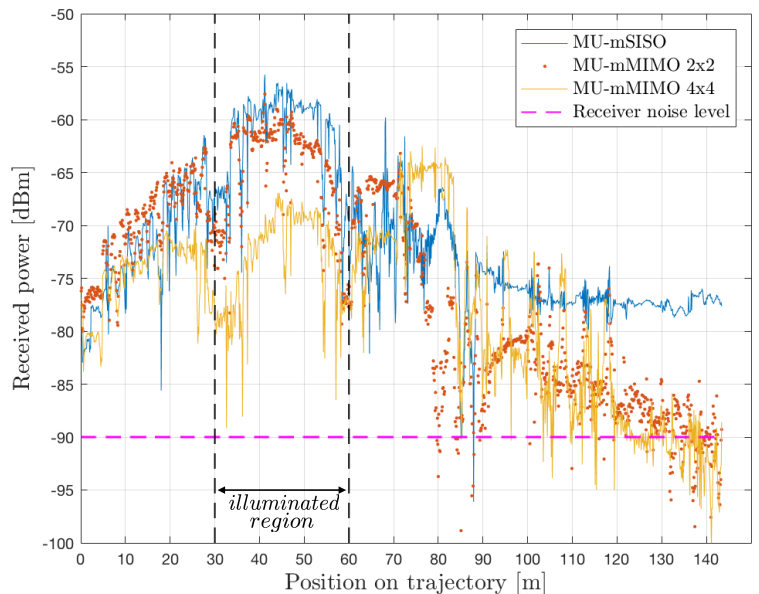

Fig. 9. Received coherent power for massive MIMO towards $P_{1}$ at each position on the trajectory with overall transmit power of $0 \mathrm{dBm}$.

\section{B. Massive Configurations}

After investigating the conventional systems, with single antenna elements, massive configurations with 64 patch antennas at the BS are examined here. The overall transmitted power is set to $0 \mathrm{dBm}$, as explained in section III-B. The beamforming capabilities of the new geometry allow to concentrate the radiated field around the user different positions.

When the received power is considered, the trajectory may be divided in two regions. First, the illuminated region corresponds to the main beam footprint, where the user vehicle is located and received power should be higher. The second can be called as shadow region and lies outside of the illuminated area, where the radiated power should be as small as possible to reduce the inter-user interference and improve the efficiency. The illuminated region has been defined as a 30-meter interval centered on the point where the BS is pointing at. In the case of beamforming towards $P_{1}$, the area where the user is expected lies between 30 and $60 \mathrm{~m}$ and, for the case of beamforming towards $P_{2}$, it is located between 70 and $100 \mathrm{~m}$.

Fig. 9 shows the received power along the reference trajectory by massive MIMO pointing towards $P_{1}$. The region of the vehicle is denoted by the vertical dashed lines. It is shown that, in the illuminated region, the received power is higher than in the rest. The peak is higher for MU-mSISO, smaller for MU-mMIMO 2x2 and the smallest power is received for MUmMIMO $4 \times 4$. This is due to the smaller directivity of the two MU-mMIMO 2x2 and four MU-mMIMO 4x4 beams, which have reduced their beamforming directivity subdividing the elements in different modules. On the other hand, the footprint of the BS is getting broader as the MIMO order increases and the illuminated region is less evident. This effect implies that, for the particular case of MU-mMIMO 4x4, the received power is maximum in the shadow region, where the shortest distance point to the BS is located, and not around the car position.

Fig. 10 depicts the received power when the target is $P_{2}$. This point is located just after the corner. Due to the focusing into this region, which is also the one corresponding to the shortest distance, the improvement is more noticeable. 


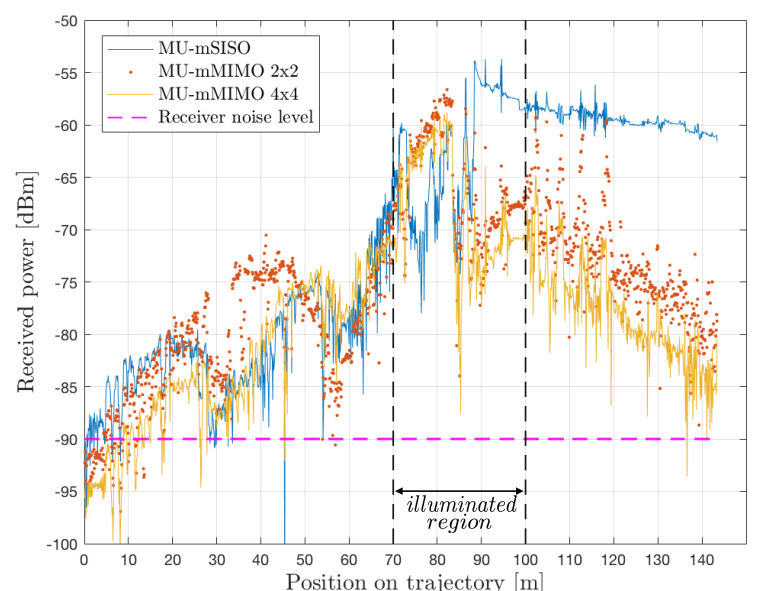

Fig. 10. Received coherent power for massive MIMO towards $P_{2}$ at each position on the trajectory with overall transmit power of $0 \mathrm{dBm}$.

Otherwise, the last part of the trajectory is following the same angular direction from the BS and the received power is just reduced by $5 \mathrm{~dB}$ from 100 until $143 \mathrm{~m}$ in the case of MUmSISO. The reason for this effect is that the BS is comprised of four patch antennas in the vertical axis and therefore the resolution and focusing capability is significantly smaller than in the horizontal axis, where the BS consists of 16 patch antennas.

The eigenvalues are analyzed for the entire trajectory for the two steered beams. In a very rich scenario in terms of multipath, values close to the ideal uniform distribution are expected $\left(\lambda_{i}=1 / N\right.$ for MIMO NxN, $\left.i=1, \ldots, N\right)$. For the case of study, there is almost always LOS and the obtained values may be not reach the ideal case.

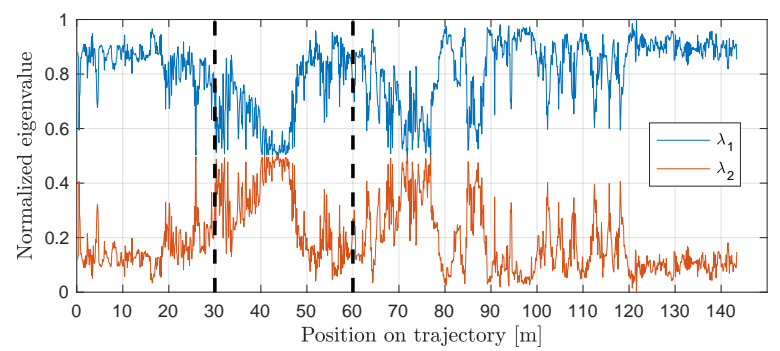

(a) Eigenvalues MU-mMIMO 2x2.

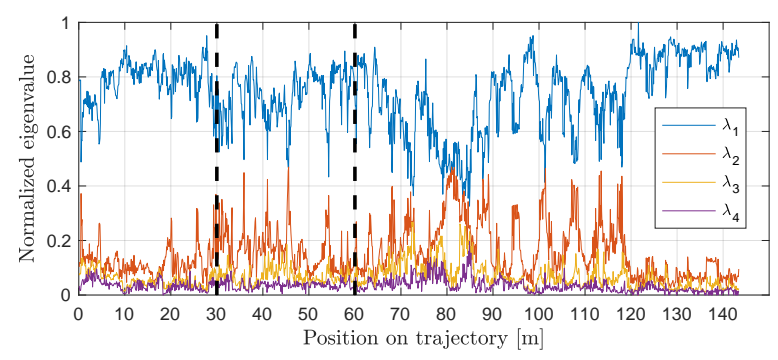

(b) Eigenvalues MU-mMIMO 4x4.

Fig. 11. Normalized eigenvalues for MU-mMIMO 2x2 and MU-mMIMO $4 \times 4$ at each position on the trajectory with beamforming towards $P_{1}$.
Fig. 11 and Fig. 12 show the normalized eigenvalues for the massive beams towards $P_{1}$ and $P_{2}$, respectively, both for MUmMIMO $2 \times 2$ and $4 \times 4$. For both reference point, it is shown that the eigenvalues are still heterogeneous. Only some points with two or even three strong paths, mainly around the corner, exhibit a distribution close to the ideal case. Nevertheless, it is necessary to take into account the received power at these points, which also has a clear relevance in the final performance.

Fig. 13a gives an overview of the average channel capacities of all massive configurations for beamforming towards $P_{1}$ and $P_{2}$. Then, the corresponding average SNR is depicted in Fig. $13 \mathrm{~b}$ and the capacity is again compared in percentage with respect to the MU-mSISO case in Fig. 13c. It can be observed that for MU-mMIMO $4 \times 4$ the highest channel capacity is reached with $220 \%$ and $280 \%$ at $P_{1}$ and $P_{2}$, respectively. The obtained capacity in the latter is higher due to its greater SNR, which is $21 \mathrm{~dB}$ and only $16 \mathrm{~dB}$ at $P_{1}$ for MU-mMIMO $4 \mathrm{x} 4$. In the case of MU-mMIMO $2 \times 2,170 \%$ at $P_{1}$ and $165 \%$ at $P_{2}$ of the initial capacity is obtained.

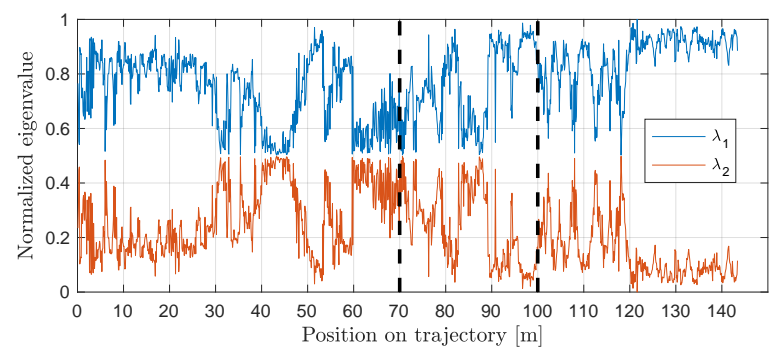

(a) Eigenvalues MU-mMIMO 2x2.

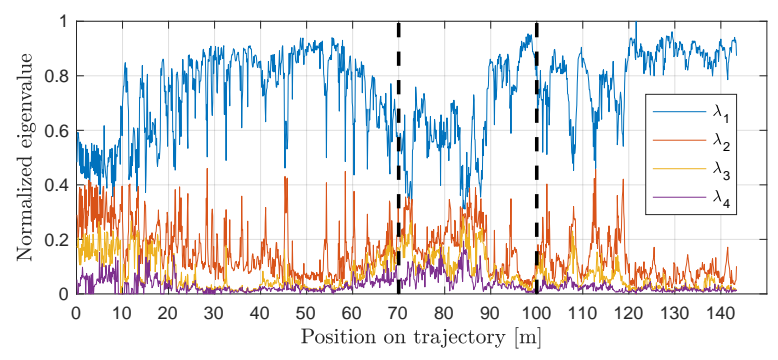

(b) Eigenvalues MU-mMIMO $4 \times 4$.

Fig. 12. Normalized eigenvalues for MU-mMIMO 2x2 and MU-mMIMO $4 \times 4$ at each position on the trajectory with beamforming towards $P_{2}$.

In any case, the transmitted power for massive BS is $0 \mathrm{dBm}$, whereas the capacity and the SNR are higher than in the conventional MIMO configurations (see Fig. 8), for which a transmit power of $10 \mathrm{dBm}$ is used. This implies also an improvement in terms of energy consumption and efficiency.

\section{CONCLUSION}

In this paper, we have modeled and analyzed the performance of different MIMO modalities in terms of their channel capacity and energy consumption. It has been shown on the physical layer with a wave propagation based model that the usage of massive MIMO instead of conventional MIMO decreases the interference and improves the energy efficiency. 


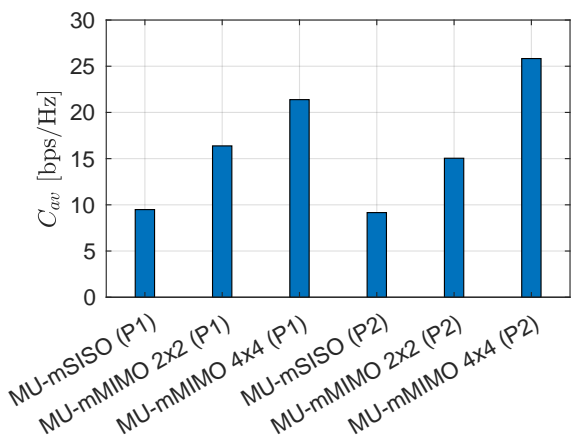

(a) Average channel capacity.

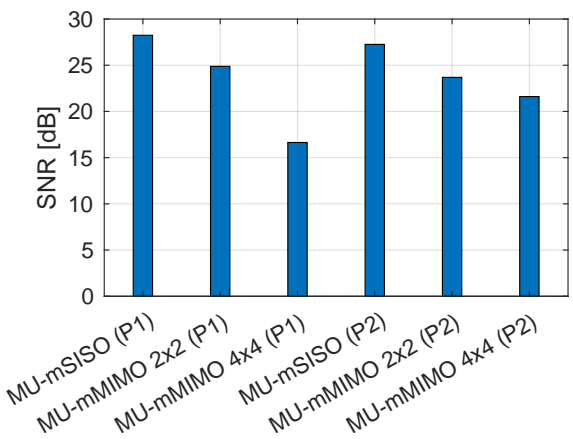

(b) Average SNR

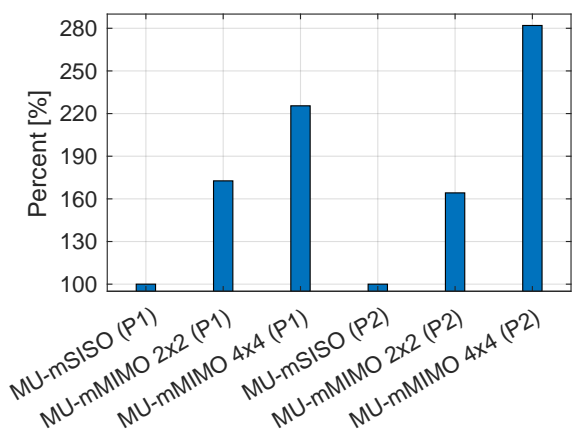

(c) Average capacity respect to MU-mSISO.

Fig. 13. Overview of the massive configuration average channel capacities and its corresponding average SNR when focused towards $P_{1}$ and $P_{2}$ with a constant overall transmit power of $0 \mathrm{dBm}$ at the BS.

Furthermore, the results suggest that the channel capacity of a massive MIMO BS can be increased by subdividing the massive antenna geometry into several channels in an urban environment. Future work will focus on the investigation of the heterogeneity of eigenvalues for MU-mMIMO $2 \times 2$ and MU-mMIMO $4 \times 4$ and the relationship between this behavior and the number of elements at the massive BS.

\section{REFERENCES}

[1] $\mathrm{Lu} \mathrm{Lu}$ et al. "An overview of massive MIMO: Benefits and challenges". In: IEEE journal of selected topics in signal processing 8.5 (2014), pp. 742-758.

[2] Erik G Larsson et al. "Massive MIMO for next generation wireless systems". In: IEEE Communications Magazine 52.2 (2014), pp. 186-195.
[3] Franco De Flaviis et al. "Multiantenna systems for MIMO communications". In: Synthesis Lectures on Antennas 3.1 (2008), pp. 1-250.

[4] Khadige Abboud, Hassan Aboubakr Omar, and Weihua Zhuang. "Interworking of DSRC and cellular network technologies for V2X communications: A survey". In: IEEE Transactions on Vehicular Technology 65.12 (2016), pp. 9457-9470.

[5] "Frequency Bands for V2X". In: ACEA Position Paper 8 (2016).

[6] Gerard J Foschini and Michael J Gans. "On limits of wireless communications in a fading environment when using multiple antennas". In: Wireless personal communications 6.3 (1998), pp. 311-335.

[7] Shintaro Shinjo et al. "Integrating the Front End: A Highly Integrated RF Front End for High-SHF WideBand Massive MIMO in 5G'. In: IEEE Microwave Magazine 18.5 (2017), pp. 31-40.

[8] I. Gupta and A. Ksienski. "Effect of mutual coupling on the performance of adaptive arrays". In: IEEE Transactions on Antennas and Propagation 31.5 (Sept. 1983), pp. 785-791. ISSN: 0018-926X. DOI: 10.1109/TAP. 1983.1143128.

[9] S. Blanch, J. Romeu, and I. Corbella. "Exact representation of antenna system diversity performance from input parameter description". In: Electronics Letters 39.9 (May 2003), pp. 705-707. ISSN: 0013-5194. DOI: 10.1049/el:20030495.

[10] R. G. Vaughan and J. B. Andersen. "Antenna diversity in mobile communications". In: IEEE Transactions on Vehicular Technology 36.4 (Nov. 1987), pp. 149-172. ISSN: 0018-9545. DOI: 10.1109/T-VT.1987.24115.

[11] S. C. K. Ko and R. D. Murch. "Compact integrated diversity antenna for wireless communications". In: IEEE Transactions on Antennas and Propagation 49.6 (June 2001), pp. 954-960. ISSN: 0018-926X. DOI: 10. $1109 / 8.931154$

[12] Björn Lindmark. "Capacity of a 2x2 MIMO antenna system with mutual coupling losses". In: Antennas and Propagation Society International Symposium, 2004. IEEE. Vol. 2. IEEE. 2004, pp. 1720-1723.

[13] Z. Mansor et al. "Impact of antenna separation on the link correlation of MIMO HetNet LTE-advanced networks". In: (Aug. 2015), pp. 15-19. DOI: 10.1109/ APWiMob.2015.7374940.

[14] Arijit De, Tapan K Sarkar, and Magdalena SalazarPalma. "Characterization of the far-field environment of antennas located over a ground plane and implications for cellular communication systems". In: IEEE Antennas and propagation Magazine 52.6 (2010), pp. 19-40. 


\section{A.5 Submitted Conference Paper: EuMW 2018}

The conference paper with the title: "5G Vehicle MIMO Antenna Capacity Based on aRigorous Electromagnetic Channel Modeling"; was submitted to the 21st European Microwave Week (EuMW) 2018 at Madrid, Spain. 


\title{
5G Vehicle MIMO Antenna Capacity Based on a Rigorous Electromagnetic Channel Modeling
}

\author{
Christian Ballesteros ${ }^{1}$, Andreas Pfadler ${ }^{2}$, Jordi Romeu ${ }^{3}$, Lluis Jofre ${ }^{4}$ \\ Antenna Laboratory, Signal Theory and Communications Department \\ Universitat Politecnica de Catalunya, Barcelona, Spain \\ $\left\{{ }^{1}\right.$ christian.ballesteros, ${ }^{2}$ andreas.pfadler, ${ }^{3}$ romeu, ${ }^{4}$ jofre $\} @$ tsc.upc.edu,
}

\begin{abstract}
Multi-antenna geometries are able to improve the performance of mobile communication systems in terms of capacity. Especially, vehicular communications are an emerging field in the context of 5G and the implementation of MIMO configurations for automotive applications requires to consider a realistic propagation environment. This work investigates the electromagnetic channel model for an urban scenario based on the achievable capacity, proposing different antenna geometries and analyzing them mounted on the vehicle. As radiating element, an UWB 3D monopole is proposed, properly designed to operate from below $2 \mathrm{GHz}$ up to $\mathrm{K}$ band, whereas this work will focus on the lowest and most critical frequencies. In particular, 3.6 and 5.9 GHz bands will be compared regarding their performance for an urban V2I environment.
\end{abstract}

Keywords - antenna design, electromagnetic propagation, vehicle, antenna arrays.

\section{INTRODUCTION}

Nowadays, with the emerging applications in the field of connected cars, there is a growing interest on investigating novel wireless technologies to achieve faster data rates, i.e., enhanced Mobile Broadband (eMBB), Ultra-Reliabe and Low Latency Communications (URLLC), and more energy efficient systems. As part of the new paradigm of mobile communications, they are the scope of research of the 5th Generation Wireless Systems (5G). All these challenges involve the use of new frequency bands, with larger bandwidths. The consequence at physical level is the need of new electromagnetic models of the channel propagation and improved designs of the antennas to cover a wide range of applications at different bands.

Many solutions are being developed in parallel regarding Intelligent Transportation Systems (ITS) and Dedicated Short Range Communications (DSRC). New standards are under discussion by regulation entities, but they can be classified in two main bands (for frequencies below millimeter waves) [1]: $3.4-3.8 \mathrm{GHz}$ and $5.9 \mathrm{GHz}$. In addition, $2.4-2.7 \mathrm{GHz}$ is a third band of interest, as WiFi and Long Term Evolution (LTE) systems are already allocated there. The necessity of a solution to operate at different sets of frequencies and supporting larger bandwidths drives this work to study the possibility of implementing the design of an Ultra-wideband (UWB) automotive antenna.

Otherwise, in terms of channel capacity, a multi-antenna configuration can improve the system performance [2] and new geometries must be considered from the initial design. In order

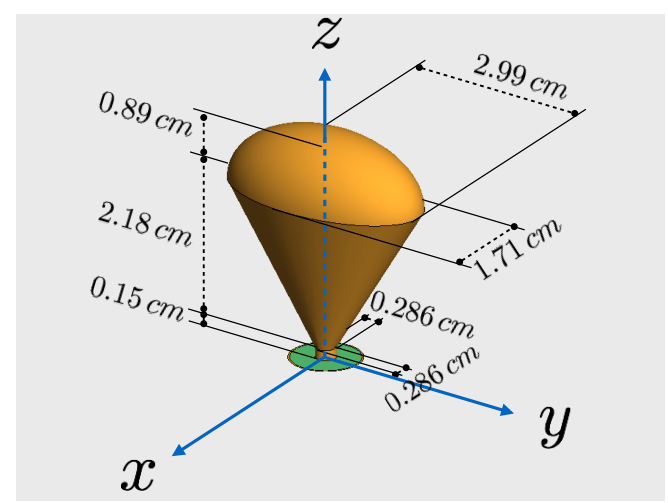

Fig. 1. UWB conical antenna with coaxial feeding on a ground plane.

to study the effect of these new configurations, the analysis of the environment for a realistic urban vehicle communication is performed. The antennas are mounted on the rooftop of a car and it is moved along a certain trajectory. Single Input Single Output (SISO) capacity is then compared to Multiple Input Multiple Output (MIMO) 2x2 and 4x4 cases.

Next sections are organized as follows: Section II details the UWB antenna design procedure, Section III depicts the different elements and the methodology used for the simulation of the antenna in a realistic urban Vehicle to Infrastructure (V2I) communication, Section IV analyzes the system performance in the simulated environment, and, finally, the work developed in this study is summarized in Section V.

\section{UWB ANTENNA}

In this section the UWB is proposed and studied in terms of its performance in free-space.

\section{A. Design and Numerical Modeling}

The first step in the process consists in designing the appropriate geometry for the antenna in order to cover the desired bands. In this case, a 3D broadband version of a monopole is chosen. Fig. 1 shows the final dimensions of the proposed UWB antenna after the optimization process. It is based on a conical structure with a hemi-ellipsoidal dome. The wide-band operation of this type of shapes has already been studied [3] and the introduction of a solid structure, instead of a printed-like antenna, allows a reduction of the total size due to the behavior of the electromagnetic waves along its surface. 
The wide frequency range of operation and the possibility of easily manufacturing this type of antennas with a commercial 3D printer are two key factors to consider. Even though, the reduced size is also a point in favour of this design, which entails an easiest implementation in real systems.

A numerical analysis of the antenna is required as initial approach to validate its performance. The chosen tool for that purpose is FEKO [4]. The simulation model includes a $30 \mathrm{~cm} \times 30 \mathrm{~cm}$ ground plane, a coaxial feeding and the conical monopole. According to current approaches [5]-[8] and the spacing constrains to place the antennas in the vehicle, usually inside a shark fin casing, the size must be optimized as much as possible, always accomplishing the initially stated goals. This optimization stage is performed using the simulation software with the following considerations:

- The Voltage Standing Wave Ratio (VSWR) is equal or smaller than 2 for the three bands of interest $(2.4-2.7 \mathrm{GHz}, \quad 3.4-3.8 \mathrm{GHz}$ and $5.8-6 \mathrm{GHz})$. It corresponds to a value of Return Losses (RLs) greater than $9.54 \mathrm{~dB}$, or S11 smaller than $-9.54 \mathrm{~dB}$.

- The maximum radius of a sphere enclosing the antenna is $1.7 \mathrm{~cm}$.

- The input impedance phase shift over the frequency must be linear to reduce dispersion [9].

\section{B. Antenna Performance}

After the optimization process, the antenna is evaluated for a wide range of frequencies. Its small size allows an adequate performance up to frequencies in the $\mathrm{K}$ band, as seen in Fig. 2a, which illustrates the reflection coefficient at the antenna input. This behavior makes the design suitable even for millimeter-wave systems. In Fig 2b, for the same frequency range, the phase of the reflection coefficient is represented. Between 2 and $8 \mathrm{GHz}$, the graph is not as linear as initially expected, but the global tendency may be considered as acceptable, mainly considering the reduced size and the wide range of frequencies covered.

The radiation pattern of the single antenna over the ground plane is shown in Fig. 3. Both co- and cross-polarized gains are represented for the two studied frequencies $(3.6$ and $5.9 \mathrm{GHz}$ ).

\section{VEHICLE COMMUNICATIONS IN URBAN ENVIRONMENTS}

In this section, the proposed UWB antenna is applied at 3.6 and $5.9 \mathrm{GHz}$ in a channel model of an urban scenario on the rooftop of a car for a V2I communication. Different multi-antenna configurations are considered: SISO, MIMO $2 \times 2$, and MIMO 4x4.

\section{A. Methodology}

In order to compare the performance of the system using the distinct geometries, capacity is used as the figure of merit to evaluate the simulation results. The channel capacity of a MIMO system may be obtained as defined by Foschini et al. [10]:

$$
C=\log _{2}\left(\operatorname{det}\left[\boldsymbol{I}_{N}+\frac{P_{T} \boldsymbol{H} \boldsymbol{H}^{*}}{P_{N}}\right]\right)
$$

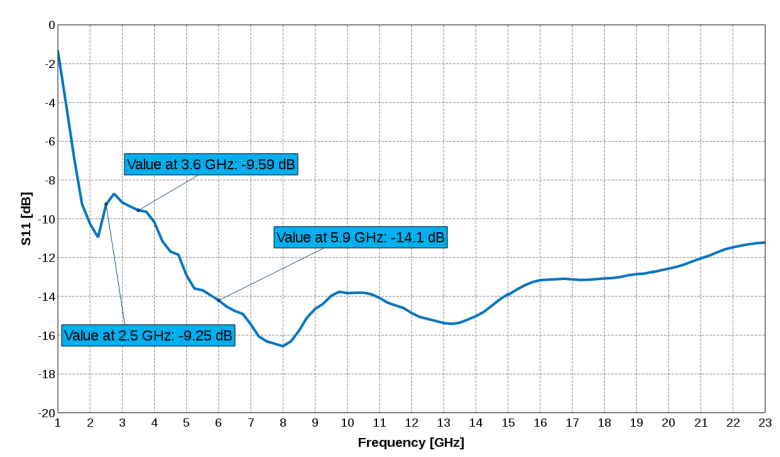

(a) Magnitude of the reflection coefficient.

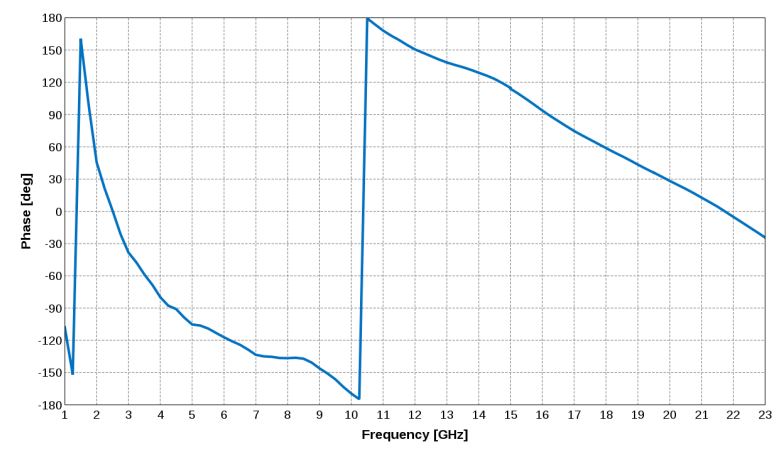

(b) Phase of the reflection coefficient

Fig. 2. Antenna performance between 1 and $23 \mathrm{GHz}$.

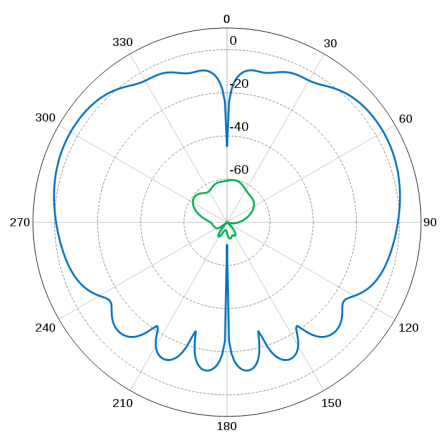

(a) Gain in $\mathrm{XZ}$ plane at $3.6 \mathrm{GHz}$

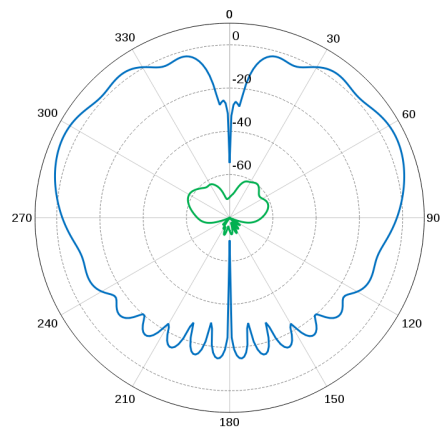

(c) Gain in $\mathrm{XZ}$ plane at $5.9 \mathrm{GHz}$

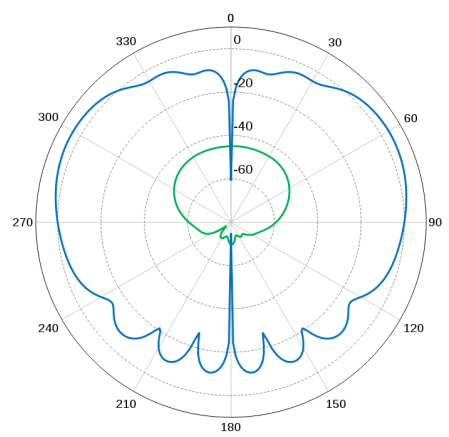

(b) Gain in $\mathrm{YZ}$ plane at $3.6 \mathrm{GHz}$

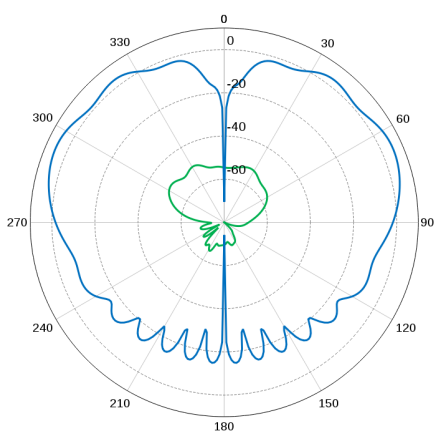

(d) Gain in YZ plane at $5.9 \mathrm{GHz}$
Fig. 3. Co- (theta) and cross-polar (phi) gain patterns in the $\mathrm{XZ}$ and $\mathrm{YZ}$ planes. 


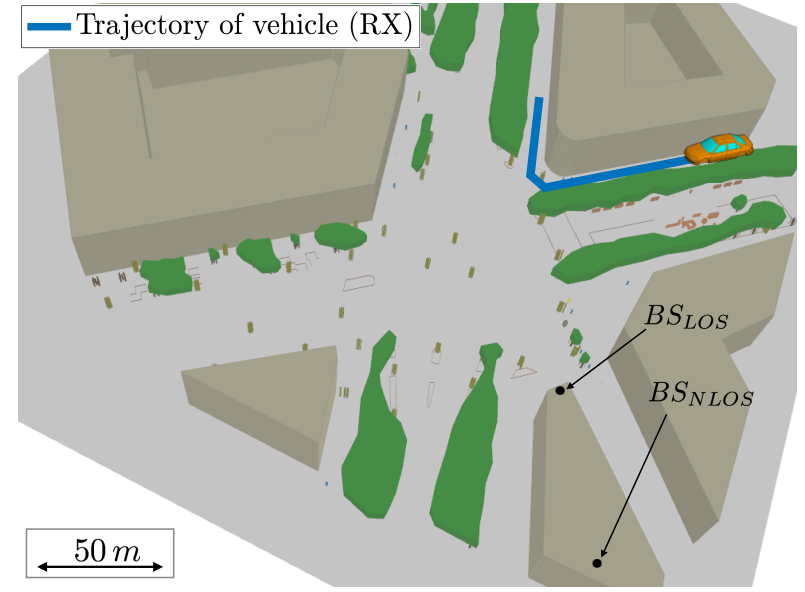

Fig. 4. The scenario of a simulated intersection in Barcelona with the trajectory of the vehicle and the two BS positions for LOS and NLOS modeled in WinProp.

where $P_{T}$ and $P_{N}-90 \mathrm{dBm}$ are transmit and noise power, respectively, $I_{N}$ is the identity matrix for $N$ receiving units, and $(.)^{*}$ denotes the transpose conjugate. The channel matrix $\boldsymbol{H}$ corresponds to the multipath relations between all input and output entries. In this case, it is obtained using a ray-tracing software tool, WinProp [11], for the chosen urban scenario.

The user (vehicle) antennas are imported from the initial electromagnetic modeling of their radiation pattern on the car and they are assumed to follow a trajectory according to a virtual route of the car. Its position is sampled and the capacity is calculated for each individual location. Finally, the average, expressed in (2) for $N_{S}$ samples, of all values is considered in the analysis of the results.

$$
C_{a v .}=\frac{1}{N_{S}} \sum_{i=1}^{N_{S}} C_{i}
$$

\section{B. The Urban Scenario}

A portion of a real downtown is selected for the modeling of a realistic V2I environment. Fig. 4 shows the intersection of two streets in the city of Barcelona, corresponding to the district of L'Eixample. The model is composed of a ground plane made of asphalt, five buildings, trees and traffic lights. Object materials are taken into account by the simulation tool in the computation. Therefore, buildings are made of concrete, trees are simulated with a wooden trunk and vegetation on top, which let the rays to pass through at the cost of additional attenuation, and traffic lights are metallic. All buildings have a height of $18 \mathrm{~m} . B S_{L O S}$ and $B S_{N L O S}$ are placed on the top of one building at height of 21 and 20 meter from the ground, respectively, with the difference that $B S_{L O S}$ has a LOS path towards the trajectory of the car and it is closer, whereas $B S_{N L O S}$ is always in a NLOS condition. All Base Station (BS)s consist of a set of omnidirectional elements placed at each of their location. One, two, or four antennas are used for SISO and MIMO $2 \times 2$ or $4 \times 4$ with a constant spacing of $\lambda / 2$ at the corresponding operating frequency for each case. The receiver, as shown in Fig. 4, is driving along a trajectory

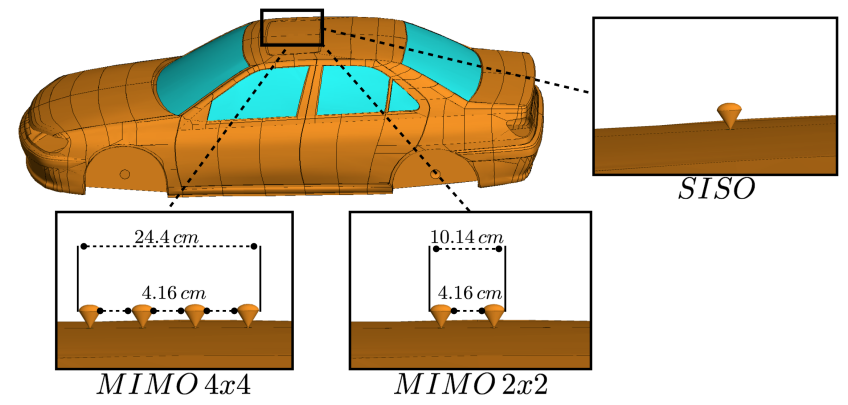

Fig. 5. Three different vehicle antenna setups with one, two, and four UWB antennas at 1.42 meter on top of the car.

(in blue) with a total length of 143.4 meter, which is sampled every 0.1 meter. Each sample represents a still position of the car.

\section{Antenna Simulation on top of the Vehicle}

Fig. 5 illustrates the three investigated antenna setups for SISO, MIMO $2 \times 2$, and MIMO $4 \times 4$ on top of the vehicle. The three geometries are aligned into a shark-fin like structure on the front part of the vehicle rooftop. The inter-element spacing is chosen to be half wavelength of the lowest frequency considered in the study, i.e., $3.6 \mathrm{GHz}$. The radiation pattern for all UWB antennas at 3.6 and $5.9 \mathrm{GHz}$ is calculated taking into account the presence of the whole car, which is modelled with metal for the body and laminated glass windows.

\section{Simulation Results}

The capacity of the distinct antenna configurations is now discussed. First, the system is assumed to work with a fixed noise level at the receiver. In second term, the channel capacity is compared fixing a mean value of the Signal to Noise Ratio (SNR) for the entire trajectory.

\section{A. Fixed receiver noise level}

In this fist approach, the path-loss of the rays for 3.5 and $5.9 \mathrm{GHz}$ is taken in to account. The utilized noise level is $P_{N}=-90 \mathrm{dBm}$ and the transmitted power is 0 and $30 \mathrm{dBm}$ for LOS and NLOS, respectively. In the case of NLOS, the transmit power is chosen to be higher due to the extra attenuation, and the final performance comparison is fair in terms of SNR at the receiver. Fig. 6 depicts the obtained average channel capacity over the entire trajectory for LOS. It is shown that, by increasing the number of antenna elements, the channel capacity is also increased for both frequencies. Even though, the achieved channel capacity for $5.9 \mathrm{GHz}$ is always smaller than the capacity obtained for $3.6 \mathrm{GHz}$ by using the same transmit power due to the fact that the path-loss is larger for higher frequencies. Fig. 7 shows the same behavior for NLOS (and higher power, as already mentioned). The main difference between LOS and NLOS is that, in the latter, the gain in capacity due to MIMO is higher, so more independent channels are assumed. Comparing SISO with MIMO 4x4 for NLOS (see Fig. 7) this lager MIMO gain is presented, by comparing the same modalities for LOS (see Fig. 6). 


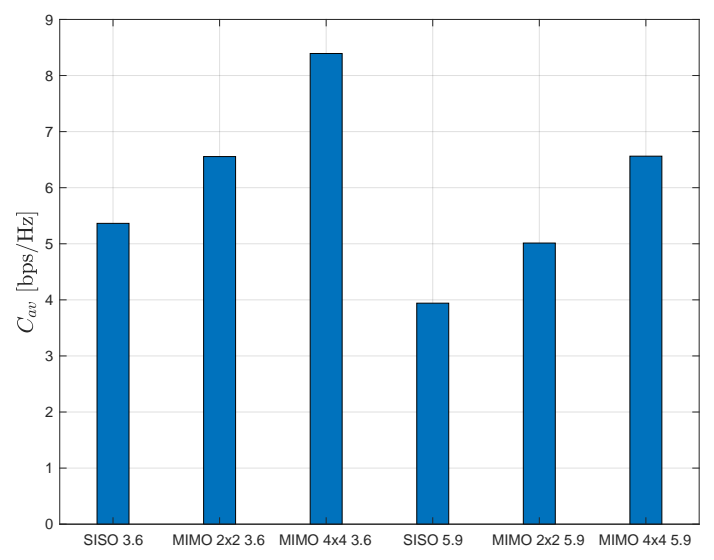

Fig. 6. Overview of the LOS average channel capacities with a constant overall transmit power of $0 \mathrm{dBm}$ at the $\mathrm{BS}$ for $3.5 \mathrm{GHz}$ (left) and $5.9 \mathrm{GHz}$ (right).

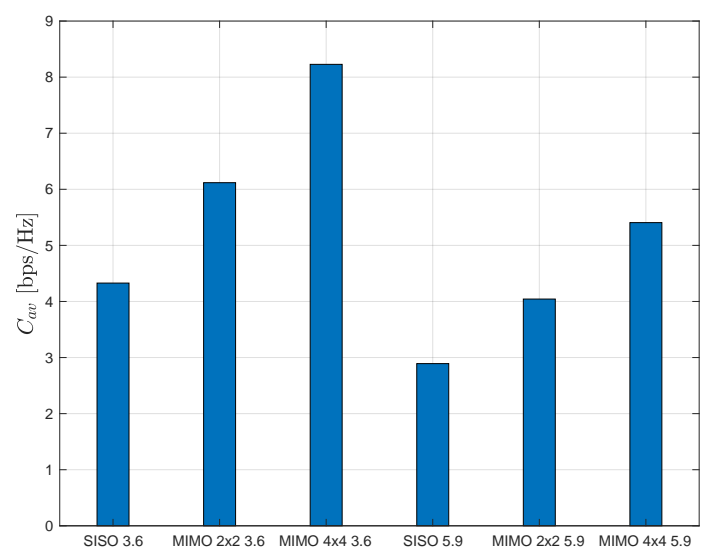

Fig. 7. Overview of the NLOS average channel capacities with a constant overall transmit power of $30 \mathrm{dBm}$ at the BS for $3.5 \mathrm{GHz}$ (left) and $5.9 \mathrm{GHz}$ (right).

\section{B. MIMO Capacity vs. SNR}

In the second approach the mean SNR is fixed over the entire trajectory. This approach still considers the fading of the received power at each receiver position on the trajectory but determine the mean SNR. Fig. 8 shows the obtained channel capacity for 0 upto $30 \mathrm{~dB}$ SNR. Fig. 8a illustrates the capacity for LOS and Fig. 8b for NLOS. Comparing LOS with NLOS, it can be seen that latter reaches higher MIMO gain. Analyzing both frequencies in terms of this gain, it seems to be that they are the same. This is reasonable due to the small difference of the wavelength, i.e., 8.32 and $5.08 \mathrm{~cm}$, which is not enough to clearly state an improvement for the given scenario. Therefore, deeper analysis is needed to investigated the richness of the channel for both frequencies.

\section{CONCLUSION}

In this work a novel $5 \mathrm{G}$ antenna is proposed, numerically analyzed and investigated at the most critical frequency

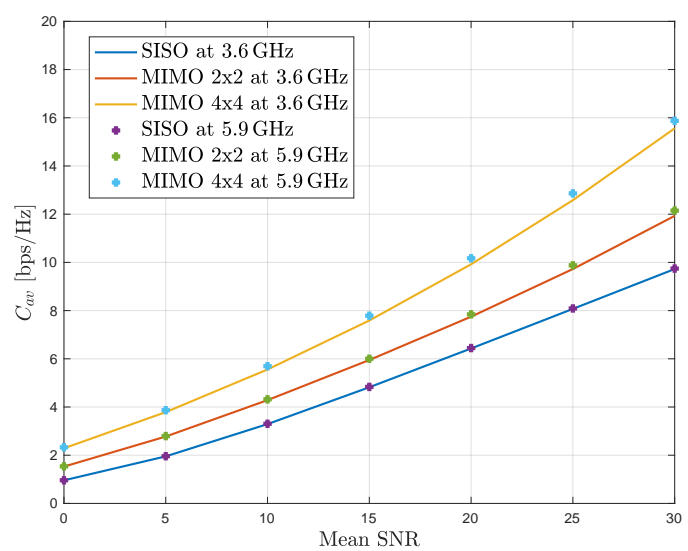

(a) Channel capacity for LOS

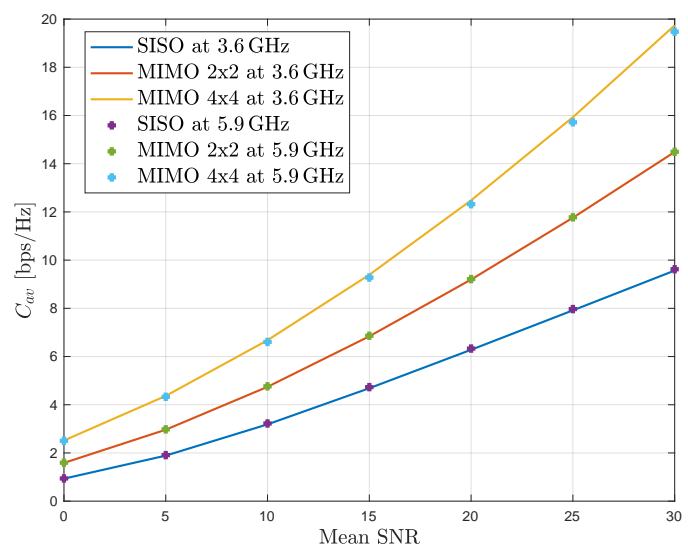

(b) Channel capacity for NLOS.

Fig. 8. Average channel capacities for distinct mean SNR.

bands for V2I communication. Different MIMO modalities are compared in terms of channel capacity for LOS and NLOS communication. The initial results suggest to use MIMO antenna configurations instead of SISO and also indicate that the obtained MIMO gain for 3.6 and $5.9 \mathrm{GHz}$ is almost the same. Further research is required and empirical measurements are expected to be performed by the conference presentation.

\section{ACKNOWLEDGMENT}

This work was supported by the Spanish "Comision Interministerial de Ciencia y Tecnologia" (CICYT) under projects TEC2013-47360-C3-1-P, TEC2016-78028-C3-1-P, MDM2016-O6OO and Altair Engineering, Inc.

\section{REFERENCES}

[1] M. Mueck and I. Karls, Networking Vehicles to Everything: Evolving Automotive Solutions, 122017.

[2] C.-N. Chuah, D. N. C. Tse, J. M. Kahn, and R. A. Valenzuela, "Capacity scaling in mimo wireless systems under correlated fading," IEEE Transactions on Information Theory, vol. 48, no. 3, pp. 637-650, Mar 2002. 
[3] D. Ghosh, A. De, M. C. Taylor, T. K. Sarkar, M. C. Wicks, and E. L. Mokole, "Transmission and reception by ultra-wideband (uwb) antennas," IEEE Antennas and Propagation Magazine, vol. 48, no. 5, pp. 67-99, Oct 2006.

[4] Altair Hyperworks, FEKO User Manual 2017, Altair Engineering Inc.

[5] M. G. N. Alsath and M. Kanagasabai, "Compact uwb monopole antenna for automotive communications," IEEE Transactions on Antennas and Propagation, vol. 63, no. 9, pp. 4204-4208, Sept 2015.

[6] C. Y. Liou and S. G. Mao, "Miniaturized shark-fin rooftop antenna with integrated dsrc communication module for connected vehicles," in 2017 XXXIInd General Assembly and Scientific Symposium of the International Union of Radio Science (URSI GASS), Aug 2017, pp. 1-4.

[7] K. N. Modha, B. Hayes-Gill, and I. Harrison, "A compact, low loss ice cream cone ultra wideband antenna," in 2006 IET Seminar on Ultra Wideband Systems, Technologies and Applications, April 2006, pp. $165-168$.

[8] M. A. Othman, M. Z. A. Rahman, M. Z. A. A. Aziz, M. F. bin Abd Malek, M. M. Ismail, H. A. Sulaiman, M. H. Misran, M. A. M. Said, and R. A. Ramlee, "Ground effect analysis for ice cream cone uwb antenna for uwb communication," in 2013 Annual International Conference on Emerging Research Areas and 2013 International Conference on Microelectronics, Communications and Renewable Energy, June 2013, pp. 1-4.

[9] M. Ito, "Dispersion of very short microwave pulses in waveguide," IEEE Transactions on Microwave Theory and Techniques, vol. 13, no. 3, pp. 357-364, May 1965.

[10] G. J. Foschini and M. J. Gans, "On limits of wireless communications in a fading environment when using multiple antennas," Wireless personal communications, vol. 6, no. 3, pp. 311-335, 1998.

[11] WinProp Software for Wave Propagation and Radio Planning. 


\section{A.6 Research Budget}

In the following, the expenses of this work are stated. Table A.1 lists the expenses of this research project. The labor costs are the expected expenses for an university employment contract. The approximated total expenses are 42,005.50 Euros.

Table A.1: Research Budget

\begin{tabular}{l|l|l|l} 
& Units & $\begin{array}{l}\text { Cost per unit } \\
\text { (Euro) }\end{array}$ & $\begin{array}{l}\text { Total cost } \\
\text { (Euro) }\end{array}$ \\
\hline Labor costs (1 month) & 12 & 3,107 & 37,284 \\
\hline Travel & 0.5 & 623 & 311.5 \\
Hotel London (Double room) & 1 & 182 & 182 \\
Flight tickets (BCN-LGW-BCN) & & & \\
\hline Research Equipment & 0.01 & 60,000 & 600 \\
Network analyzer (5 years) & 0.5 & 952 & 476 \\
Computer (2 years) & 0.018 & 3,000 & 54 \\
3D Printer (2 years) & 2 & 13 & 26 \\
Coaxial connectors for UWB antenna & 1 & 1,815 & 1,815 \\
Software packet Altair (2 licenses) & 1 & & 300 \\
\hline Publications & 1 & 300 & 350 \\
URSI Conference & 1 & 350 & 607 \\
EuCAP Conference & 1 & 607 & $\mathbf{4 2 , 0 0 5 . 5}$ \\
ADAPTIVE Conference & \multicolumn{2}{l}{} \\
\hline Total Expenses & \multicolumn{2}{|l}{}
\end{tabular}

